University of Redlands

\title{
Versioning for Groundwater Modeling
}

A Major Individual Project submitted in partial satisfaction of the requirements for the degree of Master of Science in Geographic Information Systems

by

Ruth Angenette Costley

Douglas Flewelling, Ph.D., Chair

James Ciarrocca, M.S.

December 2007 
Versioning for Groundwater Modeling

Copyright (C) 2007

by

Ruth Angenette Costley 
The report of Ruth Angenette Costley is approved.

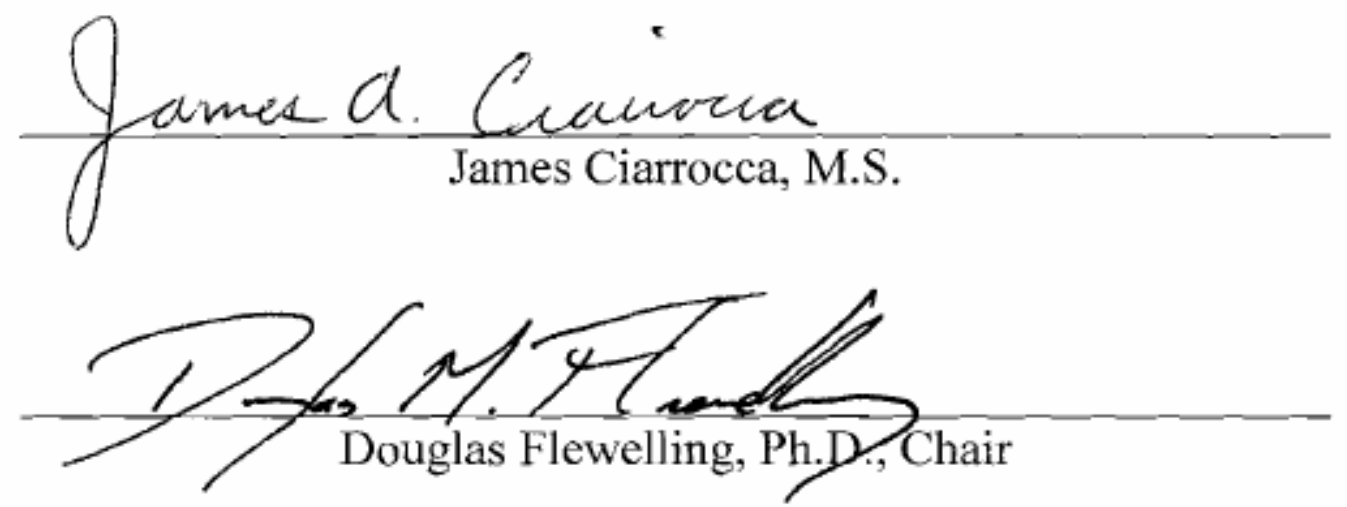

December 2007 



\section{ACKNOWLEDGEMENTS}

I suppose that one might wonder why I, a once Marine Science major, would turn in her swimsuit, towel, and love of the beach to study Geographic Information Systems and travel all the way across the country to do it. I suppose then that it is the people along the way that have encouraged me to always follow my hearts desire.

I would like to thank all of my friends and family for providing me with the courage to travel across the country to begin this program. I would like to thank, my sister, Dorothy, in particular for holding me accountable for the graduate school adventure that I had always planned. I would like to thank my former supervisor, Beverly Bures-Kirshnick for convincing me that the University of Redlands was an excellent opportunity and for giving me the afternoon off to send in my acceptance.

I am very thankful for the experience that I have had at the University of Redlands. Over the past year, I have found myself amongst some of the most wonderful people who although just classmates have become great friends.

Cohort 11. Although we were small we sure did have big ideas, and we had the best brownies. Just remember follow the Ghirardelli brand brownie mix to a $\mathrm{T}$ and add a half a bar of Hershey's symphony with Toffee, but take them out 5 minutes early.

Doug, advisor and client, thanks for giving me the wonderful opportunity to work with you. Boy, was I challenged, but I can safely say that I learned a ton.

Theresa, program coordinator, thanks for always answering my random questions and keeping this program together.

Mark, Program Director, thanks for working so hard to make the University of Redlands a great place to learn. 



\begin{abstract}
Versioning for Groundwater Models

by

Ruth Angenette Costley
\end{abstract}

Geographic Information Systems (GIS) have been found to be an excellent modeling application for groundwater systems. One such modeling application, ArcAEM, has been used in a variety of case studies and is capable of modeling head, flow, and particle tracks using the analytical element method of groundwater flow modeling. Unfortunately, the computation time of different hydrological scenarios using this suite of tools can be quite lengthy. Since multiple hypotheses are often conjectured for a particular modeled region, hydrologists often need to move between model versions to determine which one best represents the field measurements. For this reason, the ArcAEM toolbar was modified to incorporate model versioning for groundwater features, storing different parameters for features within different models. This system extends the out-of-the-box functionality of ArcSDE to track and manage model versions. 



\section{Table of Contents}

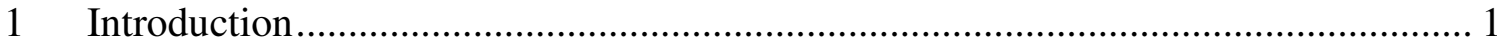

$1.1 \quad$ Problem Statement .......................................................................... 2

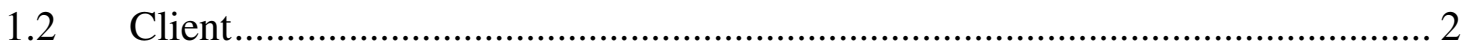

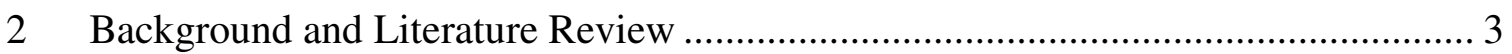

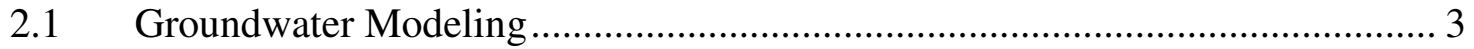

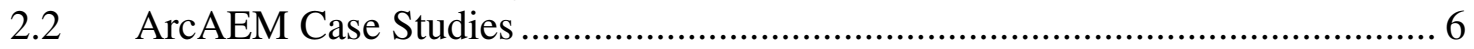

2.2.1 Development of a Numerical Groundwater Flow Model Using SRTM.... 6

2.2.2 Analytic-Element Modeling of Supraregional Groundwater Flow:

Concepts and Tools for Automated Model Configuration ........................................ 7

2.3 Other Modeling Technology ........................................................................ 7

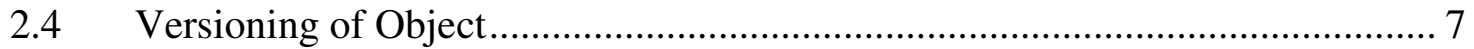

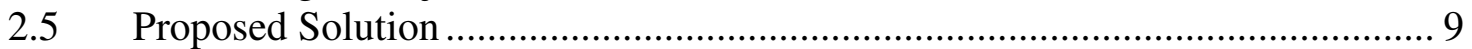

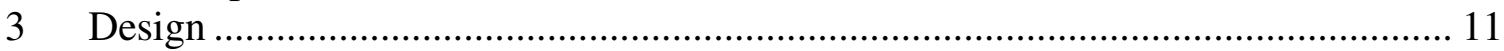

3.1 Functional Requirements ......................................................................... 11

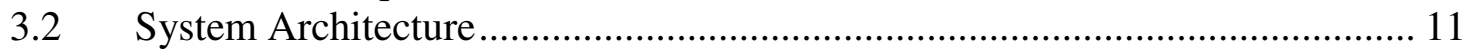

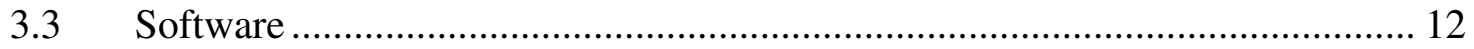

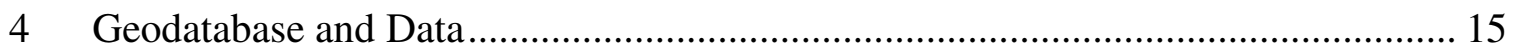

4.1 Conceptual Geodatabase Model ......................................................... 15

4.2 ArcHydro Groundwater Geodatabase .......................................................... 16

4.3 Adapting the ArcAEM Database for Versioning .............................................. 17

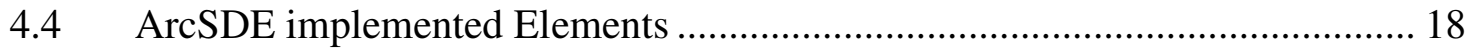

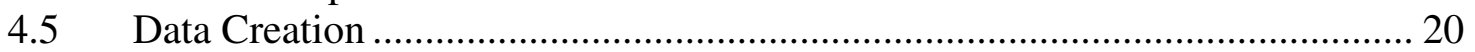

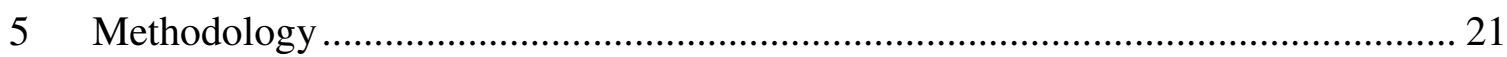

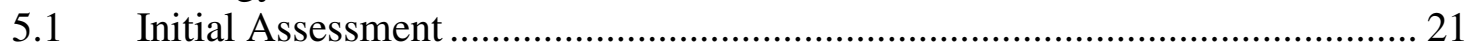

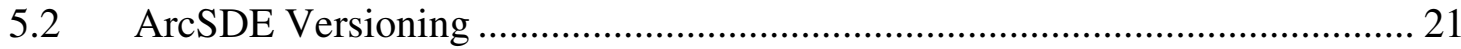

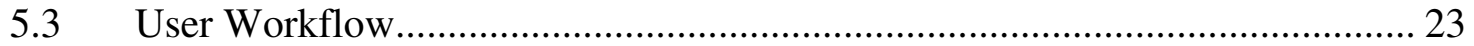

5.4 Applied Versioning Workflow Using ArcAEM v. 3.0 ……………………..... 25

5.5 Examining the Pre-Existing System ……………………………………….... 26

5.6 Programming in Microsoft Visual Basic 6.0 for ArcObjects ............................ 26

5.7 Modification of the Pre-existing system ....................................................... 27

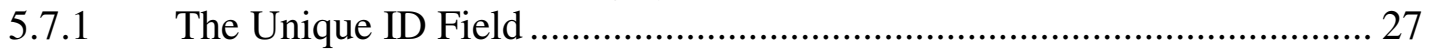

5.7.2 Create Feature Dataset Button ............................................................... 28

5.7.3 Register as Versioned Button............................................................... 28

5.7.4 Creating a Version Button ................................................................. 29

5.7.5 Change Versions Button ....................................................................... 29

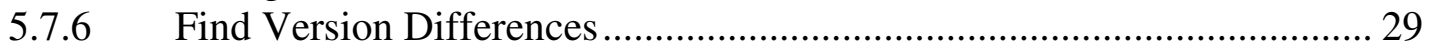

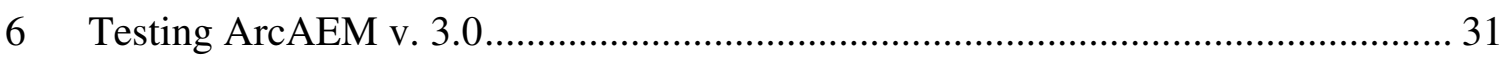

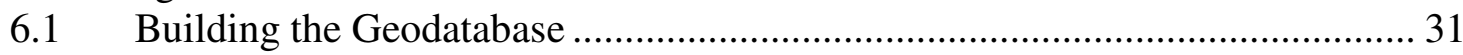

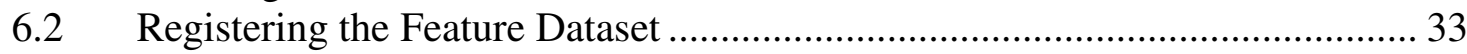

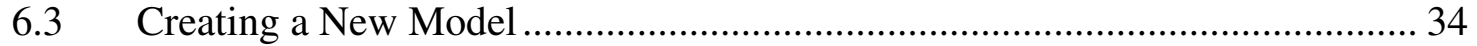

$7 \quad$ Summary and Future Work.................................................................................... 41

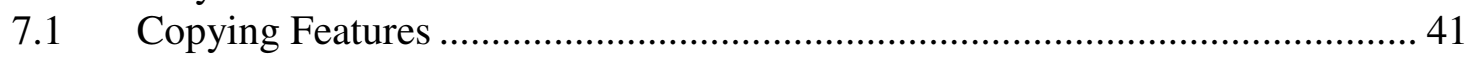

7.2 Change Accumulation.............................................................................. 42

7.3 Versioning Surfaces ................................................................................. 42 
7.4 Graphical User Interface Modification .................................................... 43

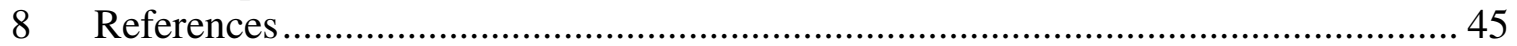

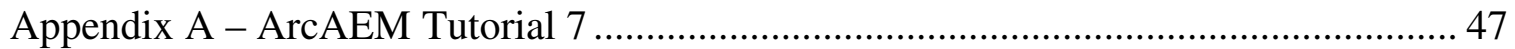

Appendix B - Create Feature Dataset........................................................................ 53

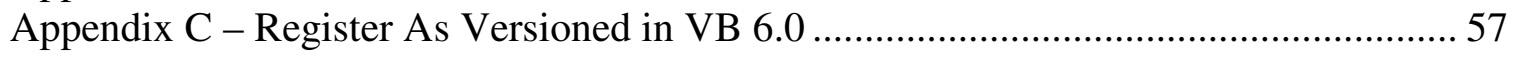

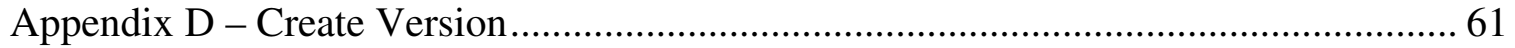

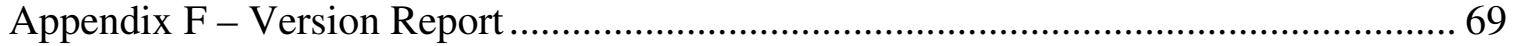

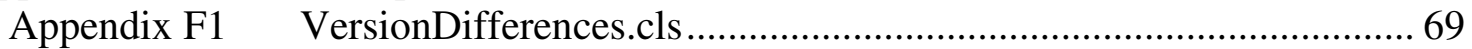

Appendix F2 modVersioning.bas ................................................................. 72

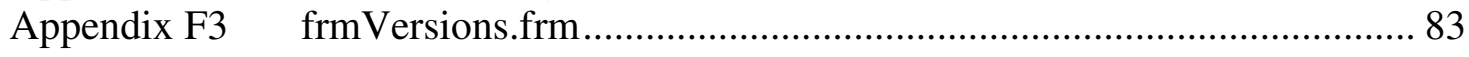




\section{Table of Figures}

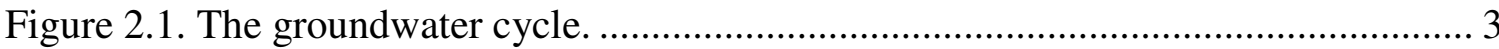

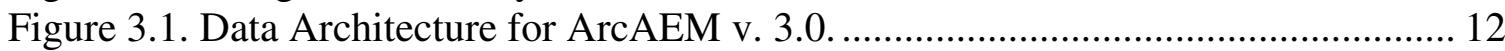

Figure 4.1. Conceptual diagram for groundwater modeling using ArcAEM. ................. 15

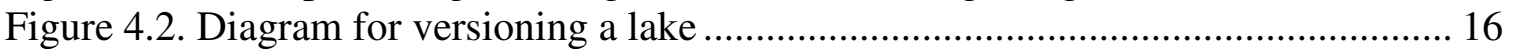

Figure 4.3. Portion of the ArcHydro Groundwater Geodatabase. ................................. 17

Figure 4.4. Participating feature classes for ArcAEM ............................................... 18

Figure 4.5. Versioning tables in the ArcSDE relational database management system ... 19

Figure 5.1 Illustration of Versioning using Lake Mathews, California .......................... 22

Figure 5.2 States within an ArcSDE versioned environment. ..................................... 23

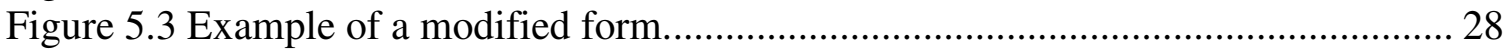

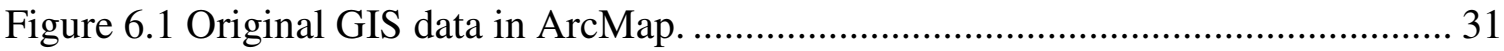

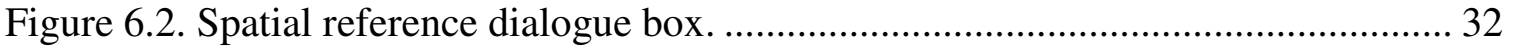

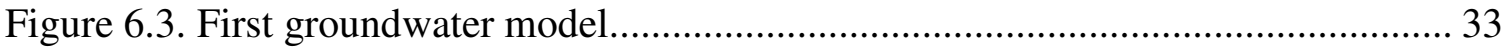

Figure 6.4. ArcAEM v. 3.0 Modeling menu .................................................................... 33

Figure 6.5. Tables Added to ArcMap for Versioning ............................................... 34

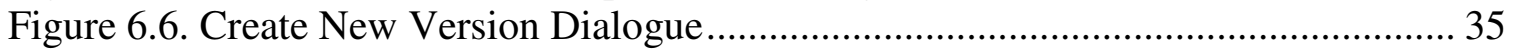

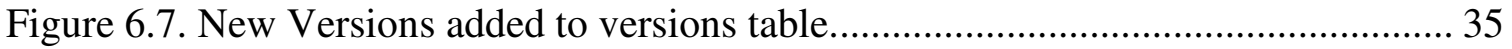

Figure 6.8 Added features in ArcMap and Adds table. ............................................... 36

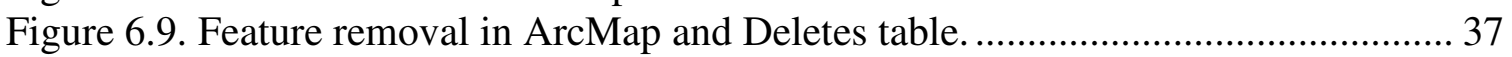

Figure 6.10. Changed feature in ArcMap, Adds table, and Deletes table....................... 38

Figure 6.11. Find Version Differences dialogue box................................................... 39

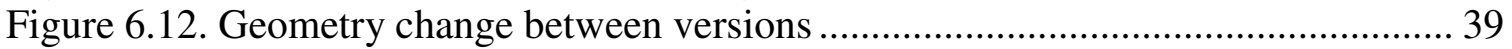

Figure 7.1 Four Primitives of Identity. ................................................................. 41

Figure 7.2 Primatives of identity within a versioned tree ........................................ 42 


\section{List of Tables}

Table 2-1.Groundwater features with respective ArcMap representations. ..................... 6 


\section{List of Acronyms}

$\begin{array}{ll}\text { ASCII } & \text { American Standard Code for Information Interchange } \\ \text { COM } & \text { Component Object Model } \\ \text { DBMS } & \text { database management system } \\ \text { DLL } & \text { Dynamic Link Library } \\ \text { ESRI } & \text { Environmental System Research Institute } \\ \text { GIS } & \text { geographic information systems } \\ \text { GNIS } & \text { Geographic Names Information System } \\ \text { OID } & \text { object identification number } \\ \text { RDBMS } & \text { relational database management system } \\ \text { VB } & \text { Visual Basic }\end{array}$





\section{Introduction}

Understanding and modeling hydrological processes is very important to preserve the environment and water resources. Groundwater makes up $98 \%$ of the available freshwater supply in the world (Schwartz \& Zhang, 2003). Unfortunately, many factors, including over consumption and pollution, affect the availability of groundwater for human consumption. As the Earth's population continues to rise, this is of great concern, since our water resources are not increasing to compensate.

The supply must be protected from human negligence, which includes the consequences of overdraft, saltwater intrusion, and point-source pollution. Overdraft commonly occurs when governing authorities are unable to adequately manage the water supply and groundwater levels are subsequently depleted (Smith, 1989). Furthermore, the depletion of groundwater within an aquifer often causes the ground to compact or subside due to the lack of physical substance amongst the porous grains of sand and gravel. This often causes flooding and damage to structures built above compacted layers (Smith, 1989). In addition, freshwater availability is also threatened by saltwater intrusion and the introduction of other pollutants such as pesticides (Smith, 1989). Point source pollution often caused by industrial sources makes the water harmful to humans.

Hydrologists often develop water flow diagrams and models for monitoring water quantity, determining availability of potable water, controlling intrusion of contaminants, depicting appropriate contaminant restoration, controlling soil erosion and flooding, and maintaining dams and other engineered facilities (USGS, 2005). Typically, groundwater is influenced by wells that draw water to the surface, streams, lakes, rivers, and aquifer boundaries. Within the context of a geographic information system (GIS), the hydrologist can model these influences through space and time.

Typical groundwater modeling techniques include head grids, flow grids, and particle tracking grids. The groundwater head can be described as the energy of water, a product of pressure and gravity, and is often measured as the water level in the area. The flow of water is described as the water velocity. Particle tracking grids show the progress of the movement of water over many years. When combined, these techniques help scientists judge the sustainability of the aquifer over time at current conditions, to decide if greater draw on the groundwater resource is sustainable; and to track contaminants through the water flow. Applications have been developed for groundwater and surface water, as well as data models to further model these qualities of water. Integrating these applications with versioning would help improve the application by adding a more scientific approach. This project is an extension of one application, known as ArcAEM, that prepares groundwater flow data for analysis by a solution engine called SPLIT, developed at the University of Buffalo. 


\subsection{Problem Statement}

The current ArcAEM application, ArcAEM v. 2, assists scientists in preparing data for groundwater models. However, the scientific process, where hypotheses are formed and tested, is often lost in the preparation due to constraints of time, data storage, and documentation. Ideally, the scientist should be able to develop and manage multiple hypotheses through various models of groundwater features. For this project, a submenu was added to create a versioning workflow within ArcAEM to maintain a data and editing history within the ArcAEM toolbar. The new submenu provides an easy-to-use versioning workflow that does not require extensive software training. This will allow scientists and engineers to develop multiple models of groundwater flow, test each model, track differences between models, and revise individual models with previous ones, thereby providing better analysis management.

\subsection{Client}

This project is a part of on going research being conducted at the University of Buffalo at New York funded through a grant with the National Science Foundation. The project client and faculty member at the University of Redlands, Dr. Douglas Flewelling, is a coprincipal investigator with, Matthew Becker, from the State University of New York at Buffalo. The University at Buffalo utilizes the ArcAEM toolbar in their research of groundwater flow analysis. To fulfill project and client expectations, methods were tested in an effort to more effectively create many iterations of a single groundwater model. This will minimize database storage, while providing an easy-to-use interface. 


\section{Background and Literature Review}

To properly model groundwater within a GIS, the real life groundwater system must be understood, as well as the similarity of the real life system to its representative counterpart. The approach taken in this research was to use real life features, and represent them in a GIS as vector features, and use the Analytical Element Method (discussed later in Section 2.1) to calculate the flow and height of the water. Versioning the features will allow multiple hypotheses to be produced. The theoretical basis of groundwater is together with data representation and versioning.

\subsection{Groundwater Modeling}

When water falls to the Earth's surface, most it collects into rivers, streams, lakes, and other water bodies. Some seeps below the Earth's surface, where it may be used by plants and other organisms, or it travels through the impermeable rock layers, through cracks and fissures. When the strata is completely inundated with water, it is termed groundwater. The flow of this water body is slow. Under the effect of gravity, water seeks to conform to the geoid; this movement is impeded by natural springs, lakes, rivers, or the ocean (Schwartz \& Zhang, 2003).

The movement may also be changed as water is often drawn to the earth's surface by wells. It is an excellent source of water, being clean and fresh due to a natural filtration system (Schwartz \& Zhang, 2003). The process of water through the water cycle is shown in figure 2.1 .

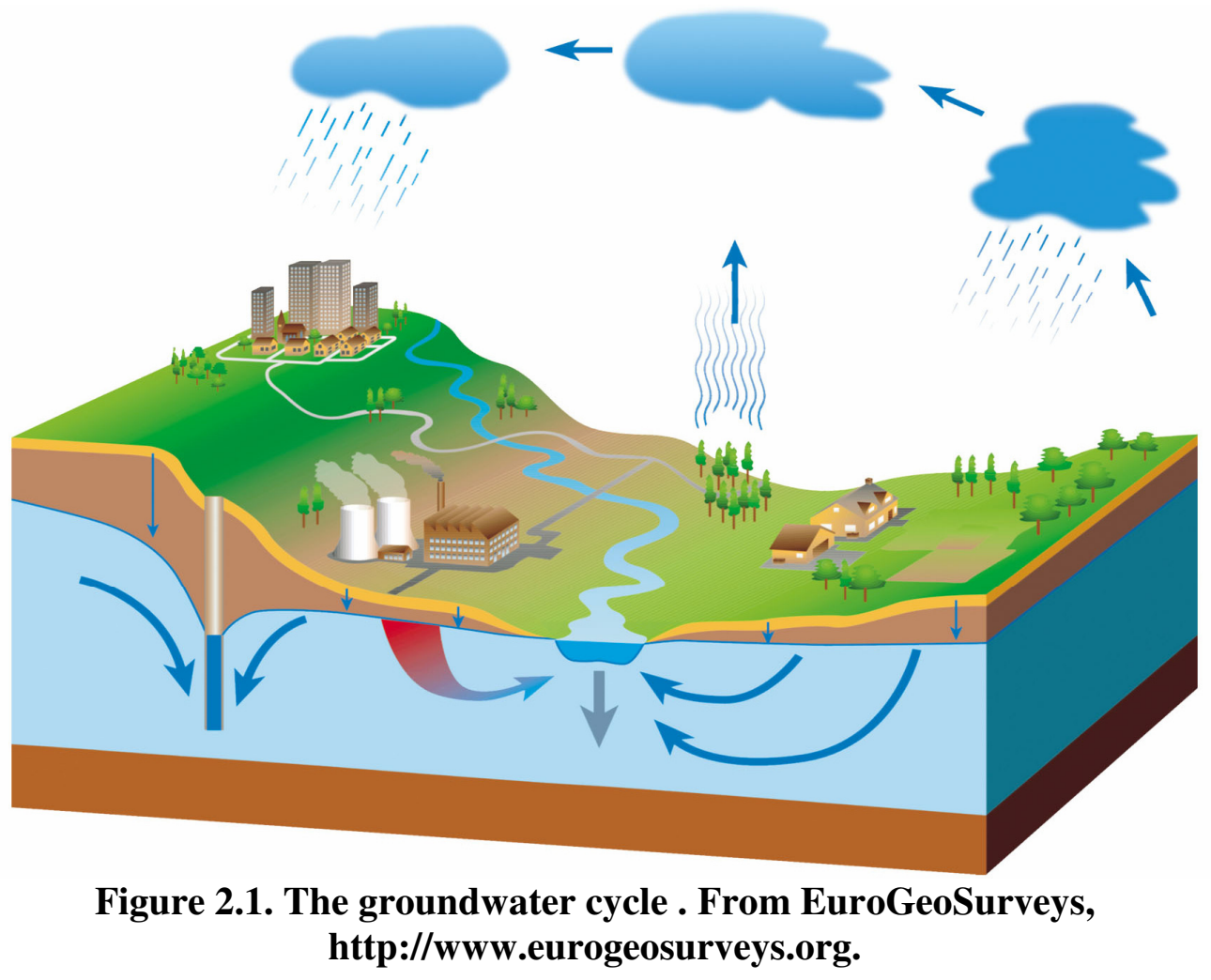


The Darcy Law can describe all low velocity groundwater flow, which is the volume discharge of water through any given sand type or other sediment with the conductivity $K$, with a given change in head $h_{2}-h_{1}$, change in length $l_{1}-l_{2}$, and area $A$ described below (Darcy, 1856 in Wang \& Anderson, 1982).

$$
Q=-K A \frac{h_{2}-h_{1}}{l_{2}-l_{1}}
$$

Equation 2.1 relates a very important principle of groundwater flow: groundwater is constantly losing energy due to friction as it interacts with the sediment around it, and it is always flowing in the direction of decreasing energy or lower head (Wang \& Anderson, 1982).

The goal of many hydrologists is to quantify and model the head and flow of water. The hydraulic head is a term which describes the energy of water flow relative to the elevation, pressure, and velocity, and is more commonly known as water depth, while the flow is the velocity of water (Schwartz \& Zhang, 2003). In the field these values may be obtained by simple measurements, but for practical purposes, one must try to conjecture these values over a given area of space and through time. The influences of groundwater are real life features such as rivers, lakes, wells, and other geologic changes, but how are we to describe these features in a GIS?

This debate is not unlike the formal debate of raster versus vector features in GIS. In this case, a raster is a continuous field across a space and a vector is an object: point, line, polygon or area. In this debate, one must consider that objects are typically manmade things, such as boundaries and buildings, where the key spatial relationship may be between the object and its neighbors. While natural things do not typically fall under this Euclidean depiction, and often the key spatial relationship of natural things is in the relations of its properties (Couclelis, 1992). A lake, for instance, has varying factors that are difficult to represent in a simple Euclidean construct. How does one depict varying depth of a lake in terms of a single polygon? The lake depth might best be described by a field or raster of data where the important relationship is the depth through space, rather than the relationship between it and surrounding objects. In this case, scientific data may best be described by rasters.

However, rasters are also imperfect in representations of objects. Due to the inherent limits of the computer systems, they must be represented as pixels corresponding to bits of data, which causes jagged edges and unrelated features. As Worboys (1994) expressed it: 'the heartfelt cry of many scientists that computers force them to make square holes for their round fuzzy edged pegs.' Worboys further emphasized the use of an object oriented approach. In this case, the varied field-like properties of objects are expressed as properties of a given condition, that is, a singular object might have many different values depending upon its state and use. The depth of the lake could be described by its maximum depth for the purpose of taking a sample at the greatest depth, or it could be described as an average depth in order to determine if a fishing boat will be 
able to travel safely through it. Given the two different sides of the debate, the best system may be the one that fits the purpose most ideally (Couclelis, 1992). Since the purpose of this system is to enable the scientist to form better models, the best approach may be the one that best allows the scientist to model their data.

In terms of groundwater modeling, three types of modeling methods that build on the Darcy Equation are currently prescribed: the finite element method, the finite difference method, and the analytical element method. The first two approaches are primarily raster based, which separates the groundwater flow equation from the head values, and they require the aquifer be fully bounded. The finite difference method separates the flow domain through a rectangular grid and computes the head at individual grid points. The finite element method separates the domain into a grid of finite elements of any shape, typically triangles or squares. The analytic element method involves identifying each groundwater feature within a system and applying a mathematical equation. This allows individual features to have varying strengths on a given region (Strack, 1989).

The analytic element method, a vector based approach, was used in this study. The analytic element method was developed by O.D.L Strack to compute groundwater flow. By this method, each individual element of a given aquifer is described by a function, where the element represents a certain condition and this representation is defined by a distinct set of coefficients given to the equation. In steady-state conditions, only these element inputs are required to calculate the flow (Strack, 1989). The typical elements used in this method are water bodies, individual pumped wells, well-fields, leakage from other aquifers, and geological formations (Csoma, 2003). This method follows the law of superposition (Strack, 1989) which asserts the summation of the influence produced by multiple wells within a given aquifer (Schwartz \& Zhang, 2003). For a given point in an aquifer, the head and flow is the sum of all the influences around it, according to a spatial weight.

Due to the object-oriented nature of this modeling approach, the analytical element method can be applied to a model with ArcGIS features, where individual elements are represented as vectors (Fredrick, Becker, Matott, Daw, \& Flewelling, 2007). The elements are composed of three different components: geometry, hydrogeological properties, and mathematical representations described by the Analytic Element Method (Steward \& Bernard, 2006).

The University of Buffalo has developed a toobar named ArcAEM within ArcGIS to develop groundwater flow models. ArcAEM uses inputs of vector data representations of the features hypothesized by the hydrologist. Table 2.1 shows the groundwater features that define the ArcAEM model and their given representations as a feature within ArcMap. 
Table 2-1.Groundwater features with respective ArcMap representations.

From Silvisesrith, 2005.

\begin{tabular}{|l|l|l|}
\hline \multicolumn{1}{|c|}{ GIS data } & \multicolumn{1}{|c|}{ Geometry type } & \multicolumn{1}{c|}{ Analytic Element } \\
\hline Well & Point & Well \\
\hline Stream, river & Line & $\begin{array}{l}\text { Head-specified river, } \\
\text { Resistance-specified river, } \\
\text { Extraction-specified river, } \\
\text { Discharge-specified river }\end{array}$ \\
\hline Lake (boundary) & Line & \begin{tabular}{l} 
Head-specified Lake \\
\hline Lake (area)
\end{tabular} \\
\hline Soil / Geologic Data & Polygon & $\begin{array}{l}\text { Resistance-specified Lake, } \\
\text { Head-specified lake }\end{array}$ \\
& Polygon & $\begin{array}{l}\text { Inhomogeneity (Zone of } \\
\text { different aquifer } \\
\text { conductivities) }\end{array}$ \\
\hline
\end{tabular}

These features are then loaded into a solver entitled SPLIT (Fredrick et al., 2007). The solver computes the hydraulic head, stream velocity, and leakage of a given groundwater system into a raster coverage, which is then viewed within the ArcGIS interface (Silavisesrith, 2007). Both ArcAEM and SPLIT can be downloaded for use by the general public from the University of Buffalo website at http://www.groundwater.buffalo.edu/software/software.html.

\subsection{ArcAEM Case Studies}

The types of applications that can benefit from the use of ArcAEM are regional, twodimensional steady-state models, wellhead protection studies, and analyses of groundwater and surface water interaction (Hunt, 2006). ArcAEM has been used in many different case studies. The following are a few instances of this research that have been cited in this paper.

\subsubsection{Development of a Numerical Groundwater Flow Model Using SRTM Elevations}

Researchers at the University of Buffalo developed a groundwater model for Vilas County, Wisconsin, near Trout Lake, to investigate the use of Shuttle Radar Topography Mission (STRM) elevations, rather than ground data, to formulate conclusions based on groundwater flow models. The lack of ground elevation data is often the limiting factor in determining groundwater flow while using conventional methods over regional scales. In conjunction with this study, the researchers plan to continue using remote sensing data such as STRM in groundwater modeling with ArcAEM (Fredrick et al., 2007). 


\subsubsection{Analytic-Element Modeling of Supraregional Groundwater Flow: Concepts and}

Tools for Automated Model Configuration

The Analytic Element Method was used by researchers at the University of Buffalo for modeling a supraregional area, the Northern Highland Lakes Region of Wisconsin. This study also geometrically simplified data to an appropriate degree of scale using methods such as the Douglas-Peuker algorithm, in order to simplify the lines and selectively choose significant features (Radideau, Craig, Silaviserith, Fredrick, Flewelling, \& Jankovic, 2007).

\subsection{Other Modeling Technology}

As the user demand for groundwater flow models increases, many modeling applications and geodatabase models are being developed to further our understanding of the groundwater system. ArcHydro, a software product developed for hydrological modeling in ArcGIS, is one commonly known modeling software for surface water applications (Maidment, 2002). This application has been used to build both a geodatabase and a set of tools to help model and analyze surface water applications. The ArcHydro Groundwater data model is also being developed to integrate both surface water and groundwater models using geospatial, temporal, and three-dimensional data (Strassberg and Maidment, 2004).

In accordance with the groundwater data model, others are being produced to synthesize different modeling techniques. One such model, developed at Kansas State University, will produce the basic inputs for both the multi-layer analytical element method and MODFLOW, which incorporates both the finite difference method and the finite element method (Bernard, Steward, \& Legrande, 2005).

\subsection{Versioning of Object}

The standard input for the features for ArcAEM is a vector representation, often a digital line graph, which depicts the features in their most original detailed form. The features are changed during the modeling process to adequately convey their nature while creating simpler shapes in order to speed up processing time. It is necessary, therefore, to generalize each element into more simple straight lines, circles, and ellipses(Fredrick et al., 2007).

Other generalization techniques for streams and rivers may include the use of the Douglas Peuker algorithm (Douglas and Peuker, 1973; Rabideau et al., 2007). In other cases, features may be changed to better suit the model. Each individual element must contain other, more specific, data which will help to formalize the mathematical equation to be used within the computation. With this in mind, it may also be necessary to store multiple types of objects and feature-specific data for a given feature, an objective that requires a method of versioning. Versioning can be described as creating different views of a given dataset without creating complete copies.

When using the analytic element method to model groundwater flow, the features themselves are generalized, open to different interpretations, and subject to change within subsequent computations of the model by the hydrologist. For a particular model, a 
hydrologist may wish to change an attribute value, remove a feature, add a feature, or change feature geometry. Currently, ArcAEM v. 2 requires the user to create a new model for each computation. Versioning within ArcAEM would help the user define different models in order to easily create multiple different models, build on individual models, and reduce database size by not storing redundant data.

Three different versioning techniques can allow for these changes. The first technique is to copy the entire database, which is neither efficient in data storage nor sufficient in the retrievable application of the model. The second versioning technique would store multiple versions of changed features, allowing the user to select features with a simple query. Using this approach, two additional fields would be created within the schema of the database: a hydrological feature identification number, and a version identification number. For each subsequent version of a feature, the hydrological feature identification number would be the same as the original feature, while the version identification number would dictate the particular version or set of versions to which the feature belongs. The third technique is to record a change journal that would list the changes made within each subsequent version. This approach would allow the user to use different aspects of particular versions, but the use of the features would require many computations. From a performance standpoint, this technique is not efficient for the database, but the hydrologist might benefit from its structure.

The third technique of versioning features was used in two cited works. In a database scheme created by Vangenot, Parent, and Spaccapietra (2002), relationships between objects were stored semantically, linking them by their identification number. Likewise, Talens and Oussaih (1993) also stored the versioned relationships in the schema of the database. The structure of the example database tracked the versions of objects through specific fields (1993).

Other aspects of database design should also be considered when applying a versioning technique. In general, the goal of a versioned database is to minimize the volume of data by storing only the representational information required to replicate particular versions of the object, versus the creation of a completely new object (Cellary \& Jomier, 1990). The objects within the database should maintain structural consistency to permit future querying of the database (Cellary \& Jomier, 1990; Tryfona \& Egenhofer, 1997). Tracking the evolution of each object and the corresponding data are also important (Talens \& Oussaiah, 1993).

Currently, ArcGIS 9.2 has incorporated versioning capabilities into the ArcSDE Enterprise Geodatabase. Within this system, multiple different versions of the same feature can be made from the original and edited as desired, leaving the original dataset unchanged. Individual versions of singular feature classes can also stem from other previously created versions. The process of versioning within this system is seamless to the end user; the tables and feature classes for a given version in ArcMap are portrayed as separate entities, although no copies of individual feature classes were created in the versioning process. The actual versioning is implemented with a series of tables in the database management system (ESRI, 2004). 


\subsection{Proposed Solution}

The new ArcAEM components support multiple iterations of groundwater models. Although ArcAEM v. 2 develops models easily through the creation of vector features, the user has to remake each model if changes are made. The modeler may want to add features, remove features, change attribute values, or change feature geometry within a subsequent version. The new system components have interfaces that allow the user to manage changes. These changes are stored and tracked for them within the geodatabase, so that individual models can be retrieved later. The new component allows the user to view each iteration of the original model in a model tree, and generate a list that will showing the differences between features. The user will be able to easily version their data without extensive database knowledge or training.

The system described is based on the commercial out-of-the-box ArcGIS 9.2 software supported with an ArcInfo level license. The ArcSDE versioning environment is used to create versions. The groundwater modeling workflow in this case requires the input of vector features, edits of vector features, and storage of feature attributes to create a file solved by an outside program called SPLIT. The current system is capable of managing this workflow, but elements of versioning were created. This project consists of revisions and extra scripting added to the current ArcAEM system, an extension of the ArcGIS software called ArcAEM. This extension is in the form of a Dynamically Linked Library (DLL), and the revisions of code were added to this DLL. This extension will be accessible to the user via a toolbar. 



\section{Design}

The pre-existing system was capable of creating groundwater models within an easy to use environment. The new system was designed specifically to not only create these models, but also improve the scientific process by versioning. This new design incorporates new system architecture, functional requirements, and software concerns.

\subsection{Functional Requirements}

The functional requirements for this system can be divided into four major categories: data storage, data access logic, application logic, and presentation logic.

Data Storage:

- The system records versions of parent features in subsequent models without copying each individual feature.

- The system reduces data storage redundancy requiring the greatest amount of normalization possible within a geodatabase.

Data Access Logic:

- The system retrieves features from previous models.

- The system retrieves entire model versions with an easy interface.

- The system opens the original features without any changes.

Application Logic

- The system adds features, remove features, change attributes of a feature, change geometry of a feature for a given model, and recall those changes for later use provided a change table.

Presentation Logic

- The system gives the user quick and easy user buttons with subsequent form boxes to add features, remove features, change feature attributes, and change geometry.

- The system features a button with a model version tree display so that the user is able to view the parent and child versions, as well as to open those versions and the original model from the interface.

\subsection{System Architecture}

The architecture for this system was driven by the need to create models compatible with the groundwater solver. The major inputs of this are the features associated with groundwater flow, which include vector features of rivers, lakes, wells, aquifer boundaries, and geological changes. These hydrography features will be edited within the ArcGIS 9.2 commercial off-the-shelf product, using the tools created within the ArcAEM extension. The vector features and attribute tables will be stored in an ArcSDE geodatabase. The data will be versioned within these database products. The ArcAEM interface creates an American Standard Code for Information Interchange (ASCII) file 
for the groundwater model from the vector features. The user will also specify aquifer conditions such as conductivity to be incorporated into this ASCII file. The groundwater solver, SPLIT, calculates the inputs from the ASCII file to produce groundwater head and flow solutions. The SPLIT program returns a solution file. The head and flow grids are created from the solution file by ArcAEM and Spatial Analyst. The described system architecture is illustrated in Figure 3.1.

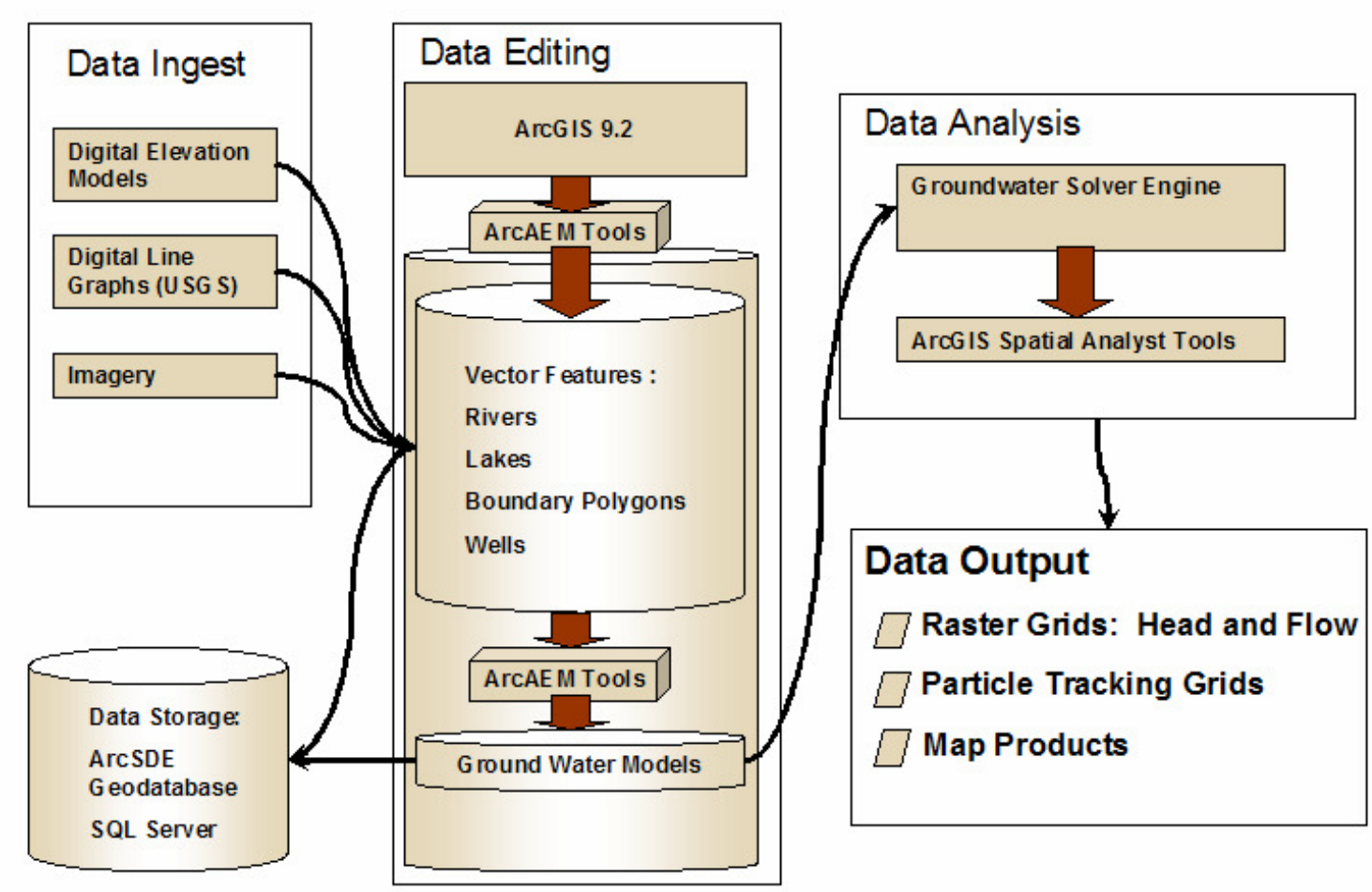

Figure 3.1. Data Architecture for ArcAEM v. 3.0.

\subsection{Software}

This system is based on the commercial out-of-the-box ArcGIS 9.2 software supported with an ArcInfo license. The groundwater modeling workflow in this case requires the input of vector features, edits of vector features, and storage of feature attributes to create an ASCII file solved by an outside program. The pre-existing system was capable of facilitating this workflow, but elements of versioning were created. This project consists of revisions and extra scripting added to the current system, an extension of the ArcGIS software called ArcAEM. This pre-existing extension was in the form of a DLL, and the revisions of code were added to this DLL. For the user, this extension can be accessed via a toolbar.

Four new sets of code were added, using the Visual Basic (VB) 6 programming environment: 
- The first component allows the user to open the original, unedited version of the model.

- The second component assists change tracking of features within models, e.g. feature addition, feature removal, feature attribute change, and feature geometry change.

- The third component allows the user to visually depict the progression of versions through a version model tree.

- The forth component allows the user to create feature datasets within an ArcSDE geodatabase. These components will add additional user interface buttons to the ArcAEM toolbar 



\section{Geodatabase and Data}

The database for this project must be capable of versioning, and then tracking the features once they are versioned. In order to maintain efficiency, the database must have reduced redundancy to ensure proper querying (Cellary \& Jomier, 1990). The highest degree of normality as possible should be achieved, while allowing the database to store feature geometry. In order to reduce the size of the geodatabase, feature classes should not be copied. Instead, tables should be created to track the differences between parent and child versions of the models. The tables and the essential versioning elements will be created. Because ArcSDE is employed, the geodatabase must be compatible with this versioning environment. A unique identifier was added to appropriately track the versioned features. This should also coordinate with other popular groundwater geodatabase models.

\subsection{Conceptual Geodatabase Model}

In order to construct the geodatabase for ArcAEM, the groundwater system must first be understood. In a given water system, many features contribute to calculating the flow of an individual particle of water, including lakes, rivers, wells, soil changes, and geological changes. All of these features must be included in the database and are the basis for the diagram in Figure 4.1. The real life features of lakes, rivers, wells, and soil/geological changes are created as vectors and made into model features. By creating mathematical models of these geodatabase features, they become Analytical Elements, which are arguments of a much larger function. These elements can therefore be calculated by an analytical solver to create a solution file which is rendered in ArcGIS to create Head and Flow raster surfaces. The analytical solver is the driver for the geodatabase. The features and attributes required for the analytical solver are reflected in the geodatabase. The data used within this geodatabase are vector features.

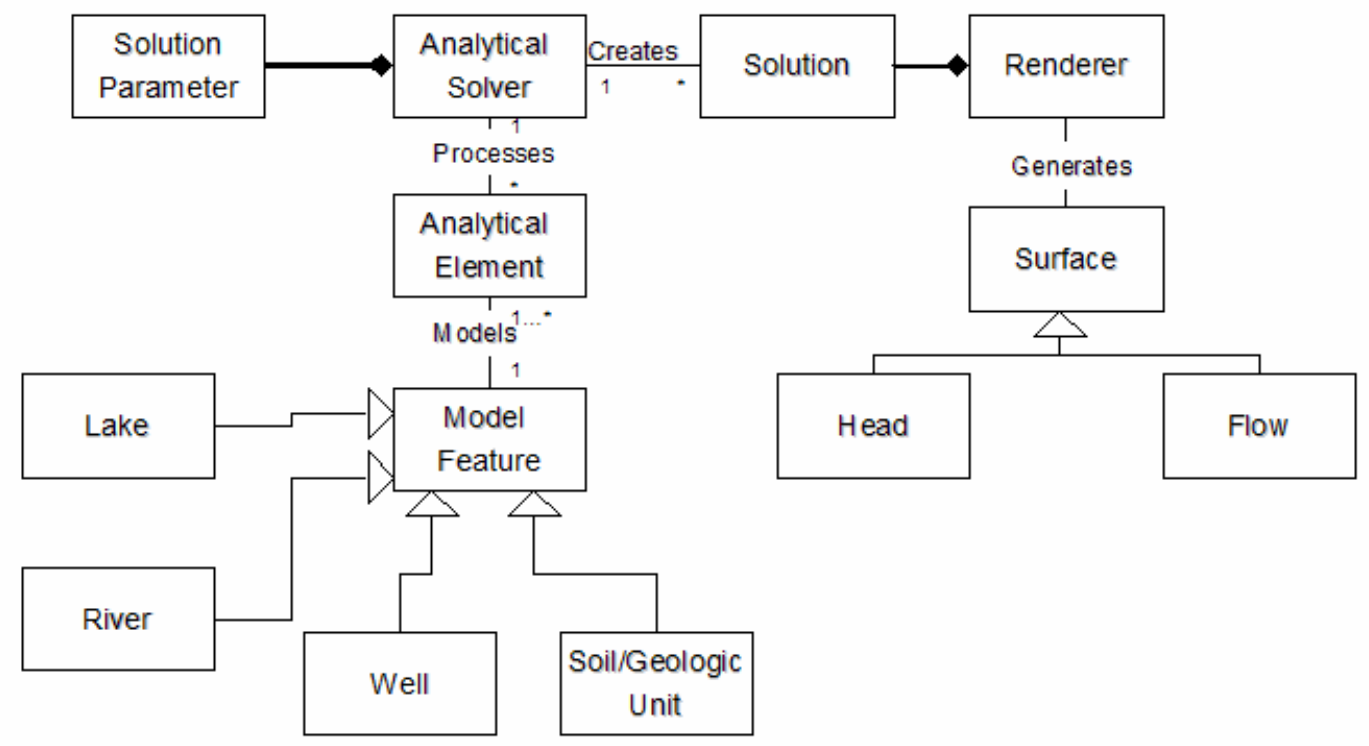

Figure 4.1. Conceptual diagram for groundwater modeling using ArcAEM. 
The new version, ArcAEM v. 3.0, changes this specification to allow for different entities of features. Figure 4.2 dictates this new aspect, where a geodatabase feature can have multiple versions. The lake in a GIS is a model feature with an original parent version of the feature. The parent, myLake: 1 , is changed and a child, myLake: 2 version is created. Many child versions can be in turn created from each parent or child.

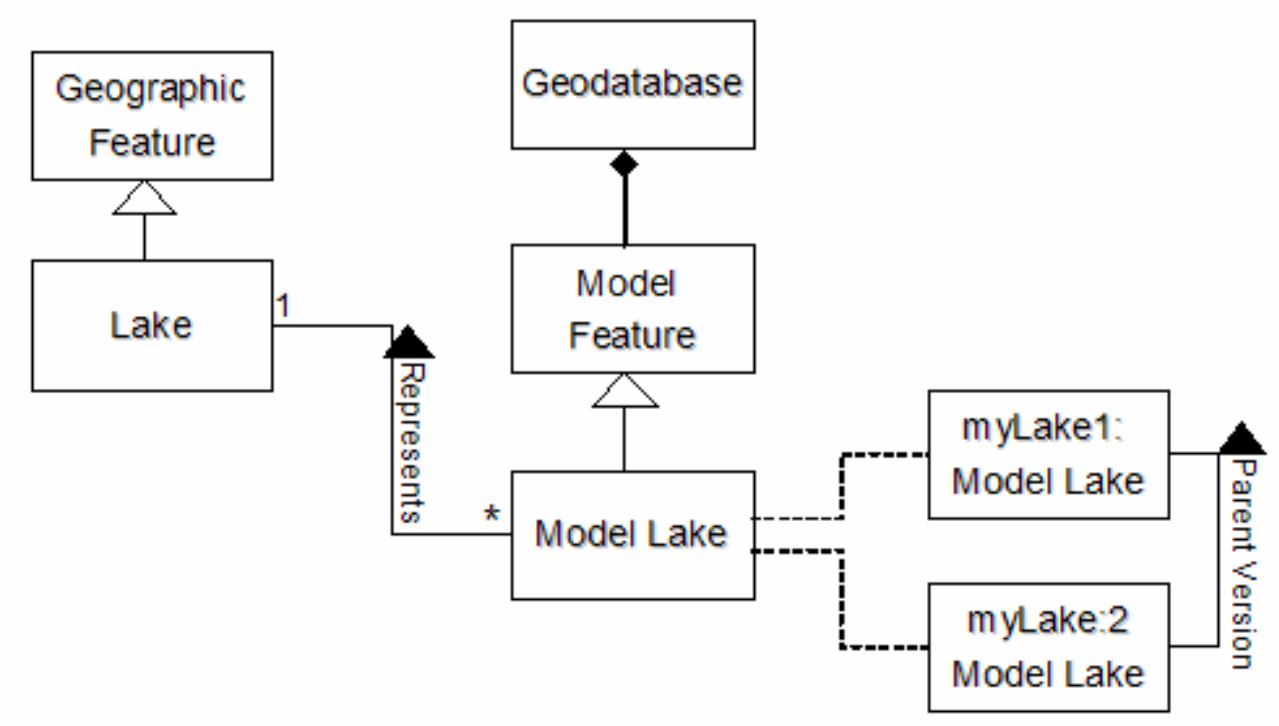

Figure 4.2. Diagram for versioning a lake.

\subsection{ArcHydro Groundwater Geodatabase}

To support user demand, the ArcAEM geodatabase should conform to the standards of the ArcHydro Groundwater Geodatabase. The ArcHydro models were built to capture the most common properties of a water system and are generalized to give an overview of water features (Arctur and Zeiler, 2004). This geodatabase supports many applications and is applicable to creating both 3D simulations and time series events. As it is, the feature classes in the ArcAEM geodatabase can be considered as types of those presented in the ArcHydro Hydrogeology feature dataset (Figure 4.3). 


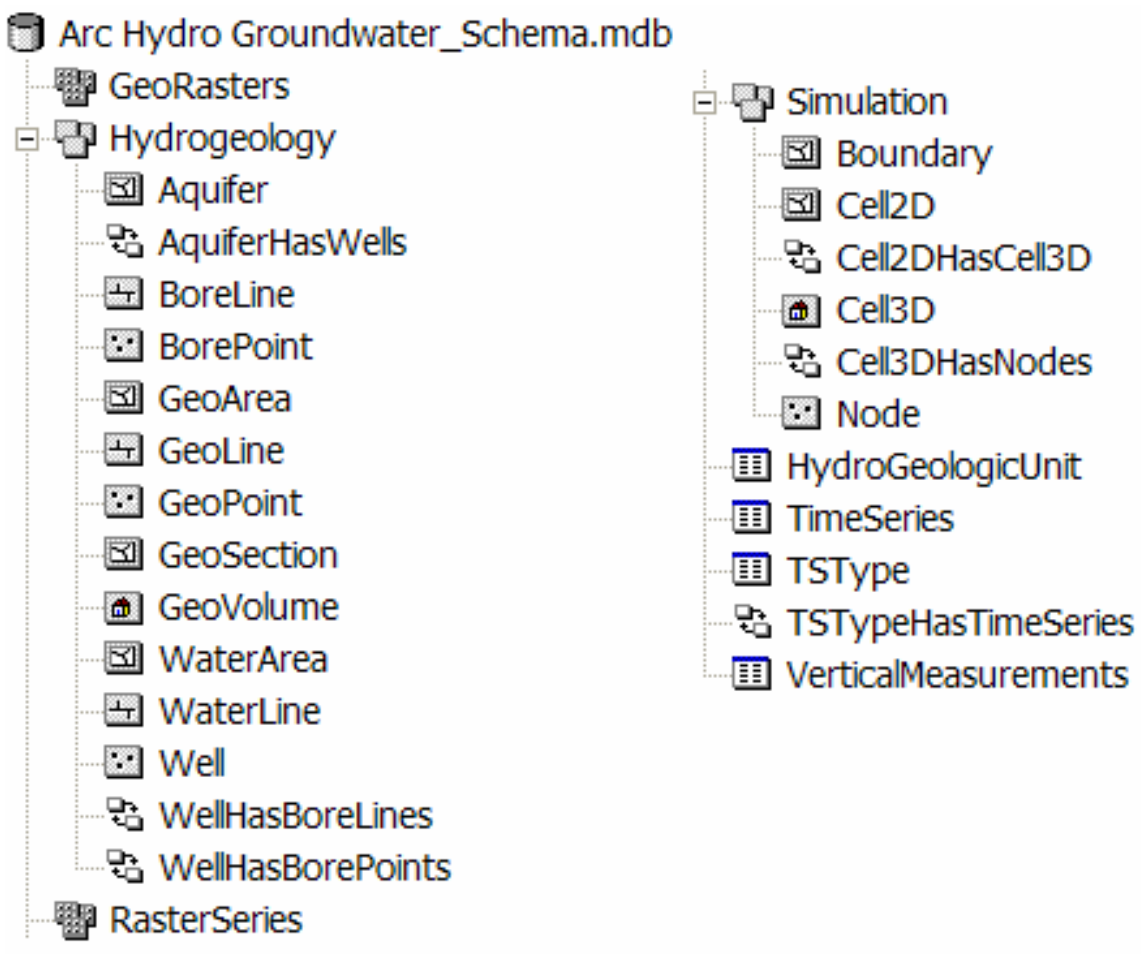

Figure 4.3. Portion of the ArcHydro Groundwater Geodatabase.

The rivers data for ArcAEM could be stored in the geoline feature class; all wells could be a part of the well feature class; and all lakes and inhomogenieties could be stored in the geoareas feature class. However, it was more practical to create a new feature dataset within the constructs of this geodatabase, which would incorporate the feature classes needed for the analytic element method of solving for flow. The ArcHydro geodatabase incorporates the United State Geological Survey and Environmental Protection Agency's National Hydrographic Dataset identification numbers for hydrofeatures and river reach codes; this naming convention is the Geographic Names Information System (GNIS). These codes were incorporated into the ArcAEM geodatabase model and serve as a unique identification number to track unique real life features among versions.

\subsection{Adapting the ArcAEM Database for Versioning}

The ArcAEM geodatabase is designed to construct the necessary contributing factors to define the groundwater flow, which are the necessary components of the analytic element model to be solved for the head and flow. Figure 4.4 depicts these feature classes combined into one feature dataset named ArcAEM Hydrography. 


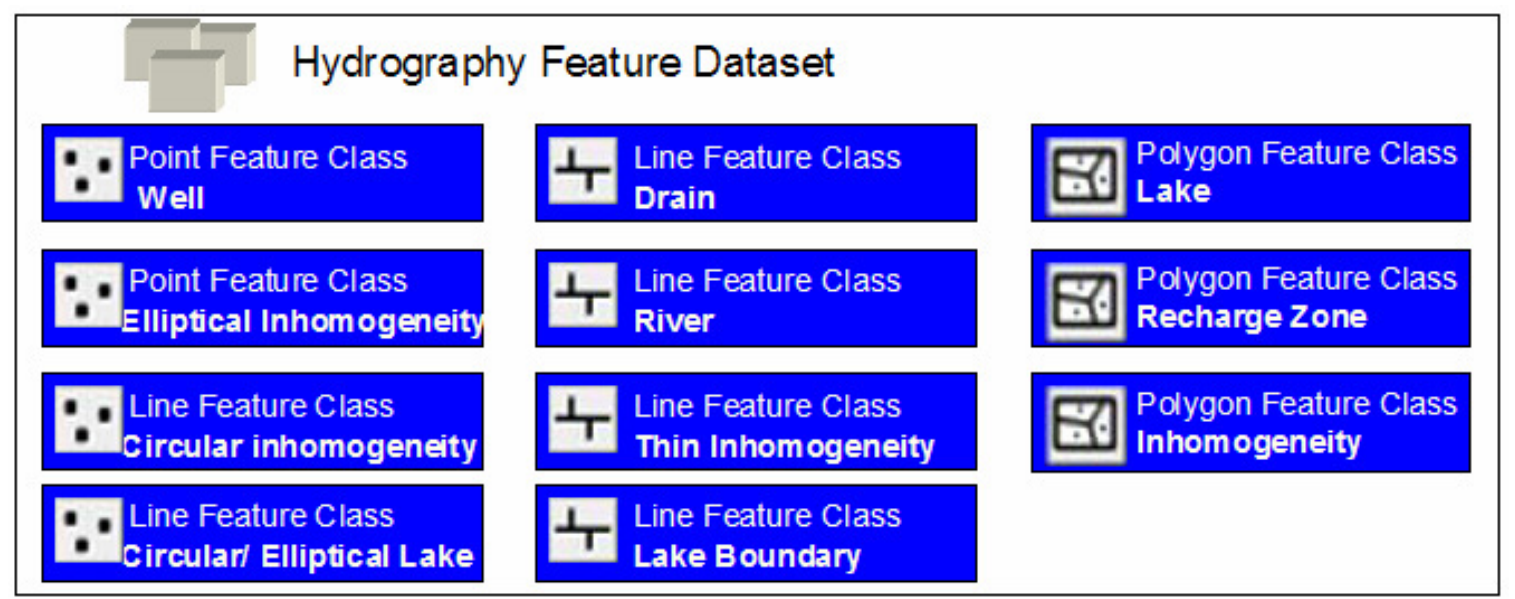

Figure 4.4. Participating feature classes for ArcAEM.

The Hydrography feature dataset contains drains, lakes, wells, recharge zones, and inhomogenieties both thin and circular. Each of these features contains a few common attributes, including a hydrological identification number, a name, a shape, and a feature ID populated by the ArcGIS software. This unique number is the formal identification number connected with the GNIS assigned to create a consistent database across all organizations of the United States. This number will also useful for querying within the geodatabase.

The other fields for these datasets are pertinent to groundwater flow and are key ingredients to the Analytical Element Model. These fields, auto-generated within the ArcAEM system, are dictated by the user with the applicable data entry form. The head attributes for the river features are often extracted from a digital elevation model, while the others are formulated from field observations and determined by a domain expert.

The feature classes for ArcAEM v. 3.0 have been placed inside a feature dataset; this is not only good practice for features that relate to one another and are of the same extent, but will also aid the versioning workflow. First, it ensures that all of the hydrography features will be of the same projection and coordinate system. Using a feature dataset will also assist in the versioning workflow. When a feature class in a feature dataset is registered as versioned, all other features will be registered as versioned as well.

\subsection{ArcSDE implemented Elements}

When a feature class is registered as versioned new tables are added to the database management system. Two basic categories sum up the newly added tables: systems tables and change tables (Figure 4.5). These tables are invisible to the ordinary user but are critical to the versioning process. 


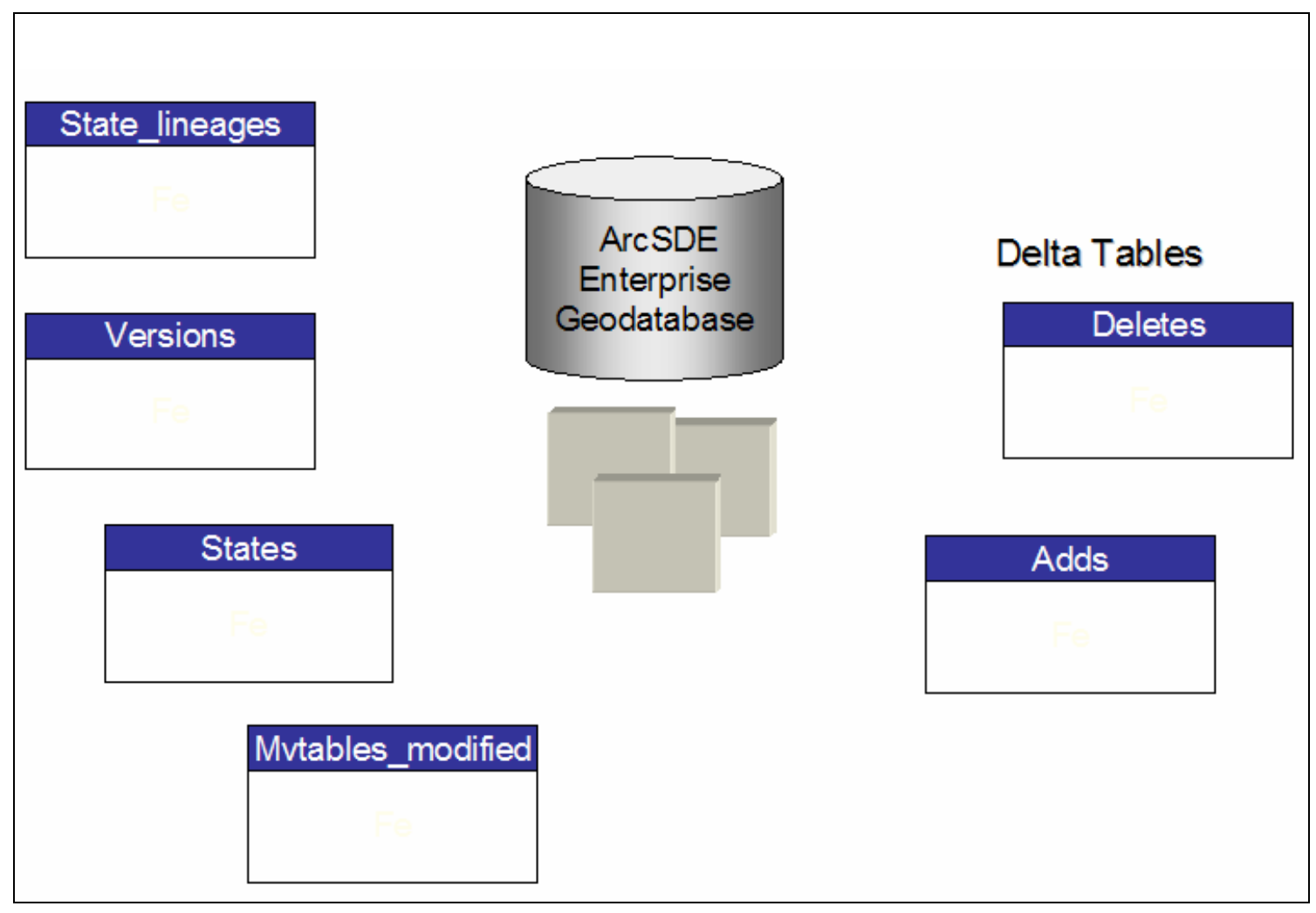

Figure 4.5. Versioning tables in the ArcSDE relational database management system.

The four system tables include: Versions, States, State_Lineages, and Mvtables_Modified. Each instance of a database that is registered as versioned has a set of these four tables:

- The Versions table contains a list of all the versions within a database. This table contains fields for the version ID, name, owner, description, date of creation, parent version, database states, and the level of accessibility: Private, Protected, or Public (ESRI, 2004).

- The States table is a list of states of the database. Every change or edit that occurs within the database creates a new state sequentially from zero regardless of the version that was edited. The states table contains the state number, creation time, closing time, parent state, and owner (ESRI, 2004).

- The State-lineages table contains a set of states for a particular branch of the version tree. The Mvtables-Modified table is the list of tables that are modified within a particular state of the database. The importance of these along with both the versions and states tables lie in the querying capacity of ArcSDE. The versions and states tables are able to identify the particular states of a particular version. The state-lineage table depicts the lineage or parent/child relationships (ESRI, 2004).

- The Mvtables-Modifed table is important for dictating which table changed within a state (ESRI, 2004). 
The modifications to the tables are stored within the corresponding delta tables, which accompany each individual feature class within a geodatabase. Two delta tables exist to handle additions, deletions, and changes. The Adds table stores all of the additions along with the state ID of each addition. The Deletes table maintains all of the removals to a particular feature class with the state ID. Each of these tables contains fields for a unique Identifier, a state ID, and all of the fields that pertain to that perspective versioned feature class or table. When a change is made to a particular feature, the transaction is recorded in both delta tables by first recording the deletion of the old attributes and then the addition of the new attributes (ESRI, 2004).

\subsection{Data Creation}

The data for this application involves vector features of rivers, lakes, wells, and geologic boundaries of a given area created by the scientist. Another source of data to be included are digital elevation models, which help incorporate hydraulic head values into the features. In general, the data for these models can be obtained from the National Hydrographic Dataset website, or digitized from base maps or aerial data. However, the purpose of this project is to incorporate versioning into a pre-existing toolbar that calculates groundwater head and flow. Since this functionality already exists, the collection of a true and accurate groundwater model is not needed, furthermore these models would need to be created by a domain expert. For this reason, simple files of rivers, wells, and inhomogenities are used as a basis to create versioned SDE features class. The changes to these models used to test the versioning functionality are generally fictitious in nature. 


\section{Methodology}

The execution of this major individualized project required researching software versioning, creating a versioning workflow, and automating this workflow within an easy-to-use environment.

\subsection{Initial Assessment}

The current ArcAEM product, ArcAEM v 2, was designed to work with the ArcGIS environment. For this reason, it was necessary to preserve this interplay, since not doing so would increase the extent of this project. Likewise, the ArcAEM v. 3.0 versioning scheme would include versioning geometry indicative of a geodatabase system, a functionality built into the ArcSDE environment. The ArcSDE environment, an integrated product with both ArcGIS desktop and ArcGIS Server, is a spatial database engine which acts as a connection between the spatial data in the GIS and the relational database management system (RDBMS) that stores the data. For this test environment, Microsoft SQL server 2005 was used as the RDBMS and was provided by the University of Redlands. The ArcSDE environment is well suited for advanced information logic, enhanced data performance, and multi-user editing, but for this project it will accomplish versioning.

\subsection{ArcSDE Versioning}

A version within ArcGIS is a snapshot of the geodatabase in which a particular version is a certain set of representations of the data that is attached to it. A single object can have many representations within the geodatabase, but for any single version just one is referenced. For the purposes of this project, a particular version is synonymous with a model. For each version produced, a new groundwater model is created. Versioning can further be illustrated within a figure tree where child versions are derived from a parent or default version. Figure 5.1 illustrates versioning of a particular feature, Lake Mathews. The original Lake Mathews is the parent of all other versions. In many cases, the original feature might be the raw data pulled into the GIS. For the first model, Lake Mathews was generalized from its original form. The second model was generalized even further. For scientific modeling, features are often generalized to speed up the processing time to compute the model. Model 3 was created in direct lineage from the parent feature. The scientist was concerned with the flow of the water, so he created a stream feature. In model 4 , the scientist changed the specification of the model, which is an attribute change. The intent of this change was to control the head of the lake, which would change the resistance variable, the opposite of the original intent. 


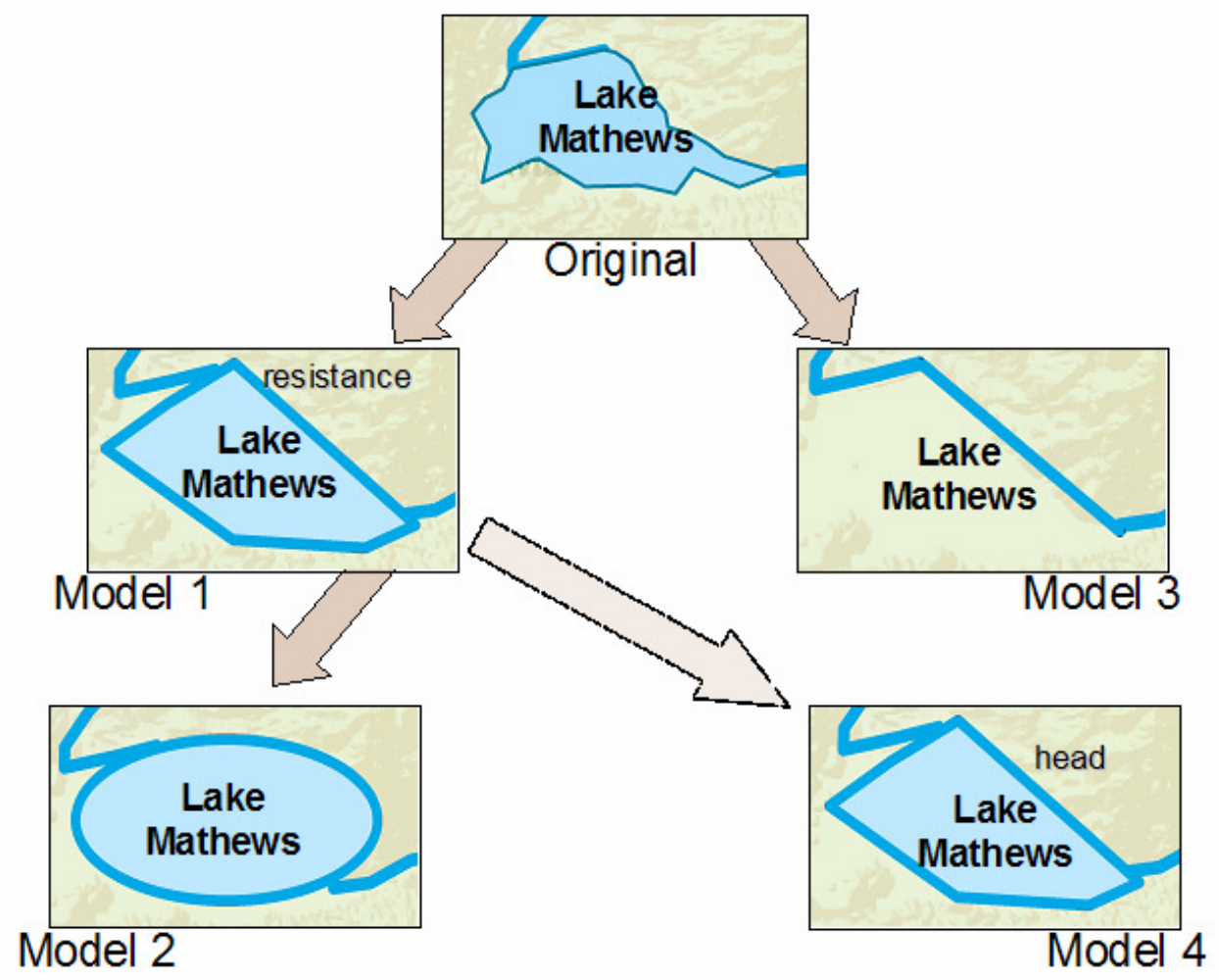

\section{Figure 5.1 Illustration of Versioning using Lake Mathews, California.}

Versions evolve from a default or original set of data. Versioning within ArcGIS is accomplished through a mechanism for, ineffect, extended long transactions, then creating tables to manage the changes within the versioned database. Long transactions allow many changes and updates to the database in a single transaction. For example when requiring money from an ATM machine, the machine first verifies that the customer exists, then it checks that the customer's account has enough money for the required amount, then it changes the customers account total to debit the requested money, and finally it debits that total amount from its own reserves before giving the customer his money. All of the checks and changes are completed in one transaction.

Versioning, however, goes beyond long transactions. For a long transaction, the database begins with a consistent state and ends with a consistent state. When the transaction ends, automated checks occur to ensure consistency. In a versioned environment, the database leaves a consistent state when the user checks out the version. When the user checks the version back in, the database is then reconciled to achieve consistency. This reconciliation is a process that the user must perform where the user must choose between changes.

In a versioned environment, tables are created to track the changes. The concept of a state is maintained within these tables. The state of a geodatabase like a particular version is a snapshot of the database, but every new edit session with one to many changes to the geodatabase is a new state. Figure 5.2 illustrates the concept of a state of the geodatabase. 


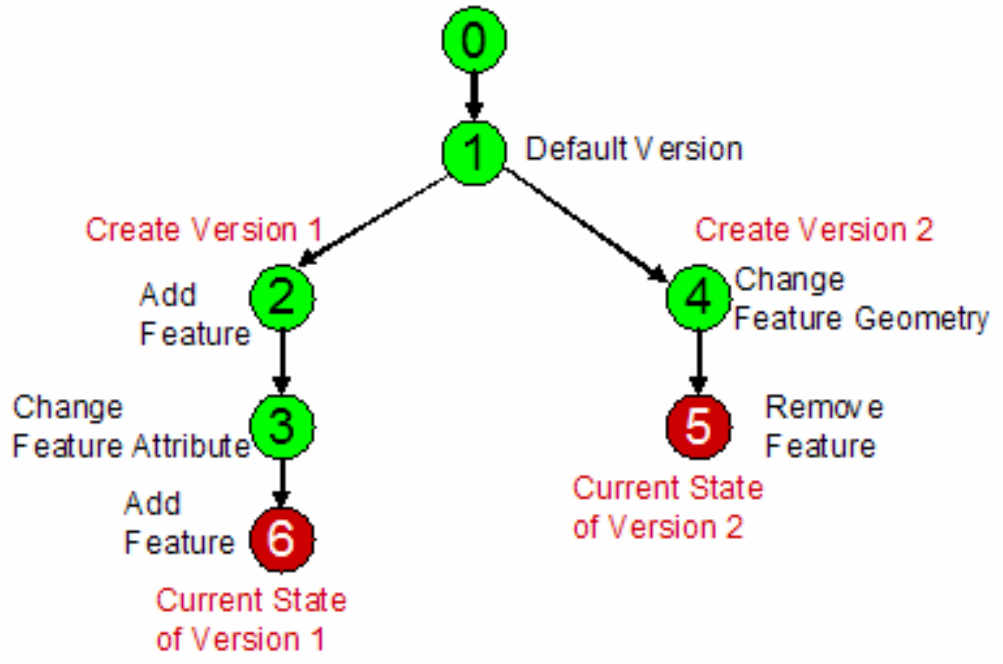

Figure 5.2 States within an ArcSDE versioned environment.

These states are numbered sequentially according to the time of execution for each edit and are stored within the states table. This means that a direct state ancestor along a tree is not necessarily the next sequential number. One branch of the tree, consisting of state parent and child relationships, is a state lineage. The state lineages table will record a series of state ids that correspond to a single lineage. The basic difference between a version and a state in this case is that a version must be established by a user.

A version of the geodatabase consists of a specific set of lineages, and to return a particular version the state lineage table is queried to identify the correct states involved. The versions are stored in another database with their corresponding state. Finally, the delta tables store the change in the geodatabase with the corresponding state number (ESRI, 2004).

\subsection{User Workflow}

After establishing an ArcSDE geodatabase instance, the user is able to create ArcSDE feature datasets, classes, and tables, and to populate them with features. These first feature classes or tables are the basis for all subsequent versions, which are only snapshots of the geodatabase according to its particular state at the time the version was saved and not copies of the original feature classes. A user can create individual versions from this default and from other versions, as well as copy features between versions. A user may post or apply changes made in child versions to the parent version counterpart, and they may reconcile or apply changes made in the in parent versions to their child versions. The versions themselves may be remain in the geodatabase for as long as necessary, or they may be deleted (ESRI, 2004).

For the scientific purpose of this project, the scientist will most likely create multiple versions adding from the default and other perspective versions. To preserve their scientific endeavors, posting and reconciling of the geodatabase may not be 
advantageous. Although this scheme of multiple versions creates a load on the geodatabase, it is necessary for the scientist to be able to create new versions, keep versions for the future, and edit all versions.

The first step to versioning is to register a feature class, feature dataset, or table as versioned. The act of registering a singular feature dataset will cause all inclusive feature classes and tables to be registered as well, if they exist at the time of registration. In accordance with the specified geodatabase, a feature dataset will be registered as versioned, which in turn registers all feature classes in the respective feature dataset at the time of registration.

When registering, the user may opt to move the edits to the base table upon reconciling and posting, or not moving the edits and allowing them to remain in the change tables. For the purpose of the ArcAEM v. 3.0 toolbar, the option to move to base will not be chosen, although the necessity of this feature is not entirely applicable since the database may never be reconciled or posted. However, if a scheme of complex editing — such as using ArcGIS topology — would be applied to the system the move to base option must not be applied. Registering a feature layer as versioned will cause the necessary versioning tables to be made within the confines of the database management system (DBMS) (ESRI, 2004).

Good practice would dictate that a protected copy of the registered version or a protected default version would ensure integrity among the database. A protected version is one which is read only to all users except for the owner of the version. Unfortunately, changing the accessibility of the default version is a property that may only be set by the geodatabase administrator. For this reason, this act will not be integrated into the ArcAEM v. 3.0 versioning workflow performed by the common user, but will be noted for the database administrator.

Once the version is registered, the user may create then additional versions stemming from this default or any other created version. Two types of versions may be created within ArcSDE: historical and transactional. An historical version is a view of the geodatabase at any given time, while a transactional version is a named pointer to a specific state of the geodatabase (ESRI, 2006).

Historical versions are not editable and are typically maintained as historical markers, so the new versions will be created as transactional versions, which will allow for future editing. The scientist can determine which version will parent any additional versions by dictating an appropriate name and access type.

Three data access types are allowed when creating subsequent versions: the protected type; the private type, which allows only the owner to view and read/write within the version; and the public type, which is freely available to all users. The public type will be suggested for newly created versions as the most compatible for new users, assuming that the database manager will restrict read/write access to the geodatabase. To protect the default, the database administrator should consider altering the default permission type to private.

The user is able to build upon their versions through the editing process and create different models pertaining to a particular version. If the user wishes to change any 
particular model, he or she would be able to create a version upon a previous one. These versions are typically depicted in a tree diagram. It is important to note that when creating a version of the geodatabase, all registered feature classes of that instance of the geodatabase will participate in that version (ESRI, 2004).

A good versioning workflow must also incorporate a few database maintenance options. First, analyzing a created feature class is important to support efficient querying of the database. This will be a built-in functionality to the toolbar available after registering the version. Analyzing creates statistics for each table within the DBMS (ESRI, 2004). A database should be routinely reconciled after edits have been saved within a version. Reconciling is the process by which edits from a parent version are merged with the child version, while detecting conflicts (ESRI, 2004). This option should never be applied to the hydrologists versioning workflow and will, therefore, not be incorporated into the toolbar.

The third maintenance option is database compression. Once all versions have been reconciled a compression process can be performed. This step removes unreferenced database states and the associated delta table rows and improves querying performance (ESRI, 2004). It should be performed on a routine basis for normal database maintenance. However, this process would require reconciling the database and will therefore not be applied despite the consequence of a slower database. Using transactional versions of this type that are not readily posted and deleted will yield a large load on the database system, but are necessary because the scientist needs to be able to create, edit, and store versions to explore a variety of hypotheses.

\subsection{Applied Versioning Workflow Using ArcAEM v. 3.0}

A prototype model was created to test versioning scenarios. First, a geodatabase instance was created on the ArcSDE server at the University of Redlands. A small set of groundwater data was loaded into the database and into a feature dataset. Another feature dataset was created to represent the non-versioned feature classes that would also reside within the database. The dataset was registered as versioned using the command available in ArcCatalog.

Many subsequent transactional versions were created using the Versioning toolbar and edited using the standard Editing toolbar. Edits created in one version could easily be copied to another version when in the proper editing environment, but a new object identification number, and a new record, would be created. It was necessary to start an edit session within a particular version, copy perspective features, stop editing, change versions, start editing in the perspective version, paste features, and end the current editing session.

The reconcile and post processes were also observed to clarify the proper use of their functionality. Edits to a parent version would not be applied to any subsequent child versions unless a reconcile was performed. Likewise edits to a child version would not be applied to parent versions unless posts were performed. 


\subsection{Examining the Pre-Existing System}

The ArcAEM toolbar was built as a dynamic link library for use with ArcGIS. The coding for the library was created in the Microsoft Visual Basic 6.0 programming environment as an ActiveX DLL with references to the ArcObject libraries. All menus, submenus, and the toolbar itself were created as class modules inheriting the Environmental System Research Institute (ESRI) ICommand properties, such as name, caption, and on-click event. The menus themselves were programmed to contain submenus calling upon the InitMenu module in ArcAEM v. 2. Modules are described as self-contained components of the system. They are often called upon in other parts of the code (Burrows and Langford, 2000). Other modules were created to be called upon while executing commands. Windows forms were created to interact with the user. The preexisting class modules, forms, modules, and overall structure were preserved when creating the new functionality. This preservation produces consistency beneficial to the users of the pre-existing system.

\subsection{Programming in Microsoft Visual Basic 6.0 for ArcObjects}

ArcAEM v. 3.0 was scripted in Microsoft Visual Basic 6.0. The original toolbar, ArcAEM v. 2, was created within this environment, and although other environments, such as Microsoft Visual Basic for .Net, framework, would have suited the advancement of this toolbar, both time and resources limited the transfer of this program. VB 6.0 is an object-oriented programming language which is both compatible and supported within ArcGIS. The leading requirement for supported languages within ArcGIS is the integration with the Component Object Model (COM) platform. All ArcObjects are COM based (ESRI, 2000).

ArcObjects can be described as libraries of software components that support ArcGIS functionality available for customization by the user. Basically, any operation or property within ArcGIS is accessible through an ArcObject. These ArcObjects are organized within object model diagram as classes, coclasses, abstract classes and interfaces. When customizing the ArcGIS software, it is necessary to become accustomed to and use ArcObjects. Using these components will change the programming environment to suite the COM platform; variables can only be declared upon object classes according to their interface, and only new objects can be created from object coclasses (ESRI, 2000).

The geoprocessing class library within ArcObjects was key to some of the functionality of the new ArcAEM toolbar. ArcGIS has a suite of geoprocessing tools that perform common tasks within ArcGIS. If these tools are used repeatedly, they can be programmed in a COM based language or modeled in ESRI's ModelBuilder for convenience to the user. For the purpose of this project, these geoprocessing tools can perform many common tasks needed for the versioning workflow and are accessible using ArcObjects within the VB 6 environment. Although these common tasks could be programmed using the functionality of the individual classes that relate to the process, using the geoprocessing tools is good practice, since ArcGIS has provided within these tools methods of error checking that may be quite complex. Stringing together Geoprocessing tools within the DLL with an easy-to-use user form creates a more effective product. 


\subsection{Modification of the Pre-existing system}

The ArcAEM v. 2 toolbar is capable of creating groundwater models. The main goal of the toolbar prior to this installment was to create an appropriate geodatabase compatible with SPLIT. The toolbar has tools to:

- Create shapefiles or geodatabase feature classes

- Create analytic elements which creates the correct fields

- Edit elements which populates the attributes

- Produce geodatabase topology

- Bring in graphics and digital elevation models

- Perform pre and post processing techniques

- Call the SPLIT solver

Modifications were made to the system to automate the versioning workflow, show versioning differences, and create a unique ID field. The additional commands to the toolbar creates a new menu called Modeling.

\subsubsection{The Unique ID Field}

The hydrologic ID field was incorporated into the Create Analytic Element Features command. Although the pre-existing system contained a unique field pertaining to a specific line, area, or point, this ID only catalogued pieces of objects, such as the reaches of a river. The addition of the ID not only provides a unique identifier for real life groundwater features to query, but also incorporates the ArcHydro geodatabase into the system. The addition of this field required modifying the Edit Analytic Element forms to retrieve the field ID from the user (Figure 5.3), and creating an additional field from the Create Analytic Element command in ArcAEM v. 2. 


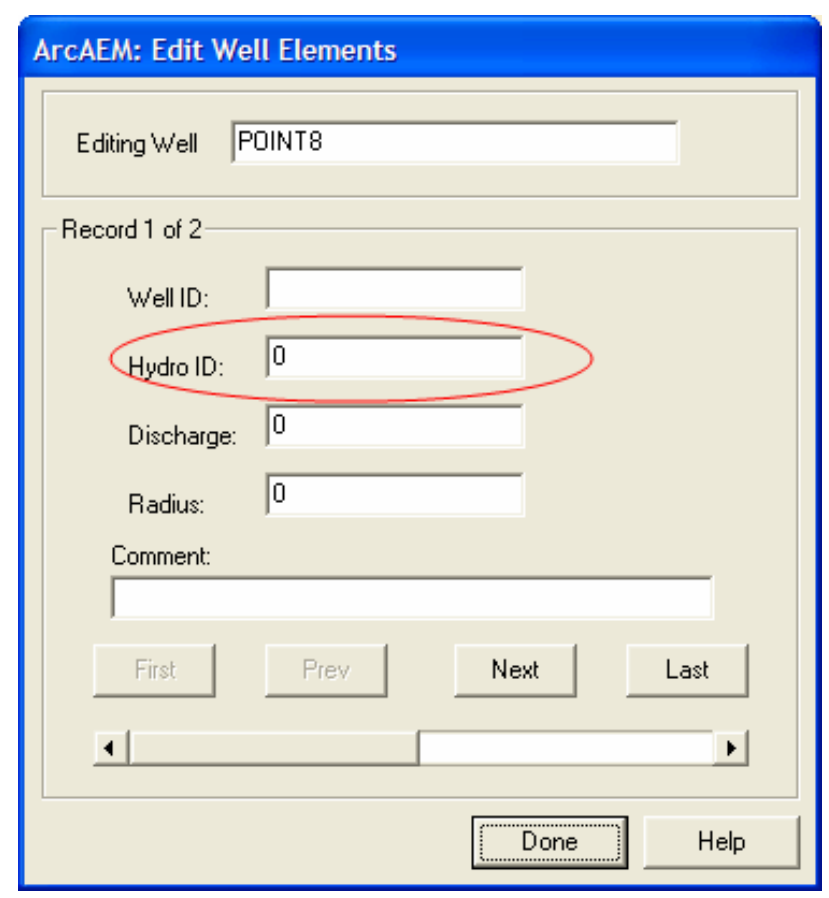

Figure 5.3 Example of a modified form.

\subsubsection{Create Feature Dataset Button}

The previous version of ArcAEM, ArcAEM v. 2 created shapefiles or feature classes within geodatabases and feature datasets. The new version of the geodatabase for ArcAEM v. 3.0 incorporates the feature dataset as a container for the versioned feature classes. For this reason, a new command, Create Feature Dataset, was added to the toolbar to create feature datasets. This new button allows the user to browse to an SDE geodatabase instance and create a feature dataset. The user can then add feature classes to the feature dataset. When the feature dataset is created, it is not automatically added to the screen. Empty feature datasets cannot be added to ArcMap. This tool incorporates the use of the Create Feature Datasets geoprocessing tool. The parameters needed for this tool are a workspace name, feature dataset name, and a spatial reference. Following the convention of the Create Feature Class tools within this pre-existing system, the spatial reference set for the feature dataset is that of the data frame. A Spatial Reference dialogue box appears for changes as needed.

\subsubsection{Register as Versioned Button}

A feature dataset or class must be registered as versioned. Registering is typically performed in ArcCatalog with a popup menu, or by registering a version from a geoprocessing tool in ArcCatalog or ArcMap. The ArcAEM toolbar incorporated this command as a submenu to the Modeling Menu. This command operates with the selected feature dataset or feature class within the source tab's table of contents and will register it as versioned without the option to move to base, and analyze business tables. The script calls the two perspective geoprocessing tools that each require the dataset input parameter. In order to diminish user error, this button is disabled for non-versionable workspaces and registered datasets. After registering as versioned, this button analyzes 
the business table, which creates statistics for the table and increases efficiency for querying within the table.

\subsubsection{Creating a Version Button}

The Create Versions submenu to the Modeling menu creates additional versions of the current version state. The user must specify a corresponding name and access, and can request to have the new version changed to the current on screen version. This button calls the Create Version command readily available in ArcMap on the ESRI Versioning toolbar. Using the pre-existing command will allow better error handling and ensure that any additional versions of ArcGIS software will permit the use of this button. This button is disabled when non-versioned workspaces or features are selected and when unregistered datasets are selected.

\subsubsection{Change Versions Button}

The submenu Change Version button allows the user to choose which version of the geodatabase is being viewed so that another version may be edited, analyzed, and compared to other versions. The currently viewed version of the database is a property of the SDE connection (ESRI, 2004). In order to change version, the property within the SDE connection must be changed. This button will also call a common ArcMap command, from the ESRI Versioning toolbar. Using this command will guarantee that proper error checking is performed and that the button will be compatible with future releases of the software. This button is disabled when non-versioned and non-registered feature classes, feature datasets, or workspaces are selected

\subsubsection{Find Version Differences}

The Find Version Differences dialogue box is an important aspect supporting the scientific process, reporting the differences between each two versions, and is a submenu to the Modeling Menu. The user interface displays both a version tree and a feature dataset tree, which allows the user to select two versions to compare, and the associated datasets to compare. Once the appropriate selections are made, the Generate Report command lists the differences in an accompanying list box. The reported differences are products of the IDifferenceCursor ArcObject connected to a given version. The differences are displayed within a list box. If the difference is a feature within the map, the user can select, unselect, pan, and zoom to the feature.

Geometry changes are also listed within this interface and, when selected, the geometry of the changed feature may be displayed in the ArcMap active frame. The code for the dialogue box was provided by the ESRI developer's network as a developer sample. The class module, form, and module code were copied into new ones within ArcAEM and modified to be compatible with the existing form, and to conform to its new function within ArcAEM. A new corresponding form object was created that matched the form code and the corresponding object.

This dynamic environment allows the user to compare the differences between two versions by selecting the respective version names and datasets to compare, and generating a report in a list view. The form dictates the changed object identification number (OID) and what the change was, whether an addition, deletion, change in 
attribute, or change in geometry. The user will then be able to zoom to those features and select them as desired.

\subsection{Tool Summary}

Once a geodatabase instance has been established within ArcSDE, the Modeling submenu will help the user register, create, switch between, and show differences between versions without adding additional toolbars or using ArcCatalog. This provides an easy-to-use interface to automate the complicated versioning workflow for the common user. Most of the design goals were met, however two commands were not created. First, the user will not be able to change the permission of the default version. This option is accessible only to administrators of the version, so this was not included in the submenu. Secondly, the user will not be able to add features that are maintained in other versions, functionality that could be thought of as a feature copy. A copy command was attempted, but the command created new features with new OIDs. The ideal copy would have maintained the old feature with the old OID, and only updated the feature attributes or geometry. This type of copy does not conform with the system requirements. 


\section{Testing ArcAEM v. 3.0}

Within this enhanced system the user completes a new modeling workflow. New models are created by adding, removing, and changing features. These models are also compared against one another to yield a list of version differences. To test these processes, a small set of data from the ArcAEM tutorial (included in University of Buffalo ArcAEM package) was used. In addition to the typical ArcMap setup in ArcGIS 9.2, the ArcAEM v. 3.0 toolbar needs to be displayed by using the Customize menu in ArcMap. An ArcSDE enterprise geodatabase is required to use the modeling portion of this toolbar, but most of the functionality will work with shapefiles, file geodatabases, and personal geodatabases. The user must also have read/write access to a geodatabase instance.

\subsection{Building the Geodatabase}

Modeled features in a GIS were created from raw GIS data. In this case, the data was compiled from a small set downloaded from the ArcAEM website. This first set of data is part of St. Lawrence County, New York (Figure 6.1). The original data set includes features such as lakes, rivers, roads, and boundaries. The original raw data can remain as either shapefiles, file geodatabase features, or personal geodatabase features. The data may also be brought into ArcSDE as non-versioned feature classes. These nonparticipating feature classes are not to be registered as versioned.

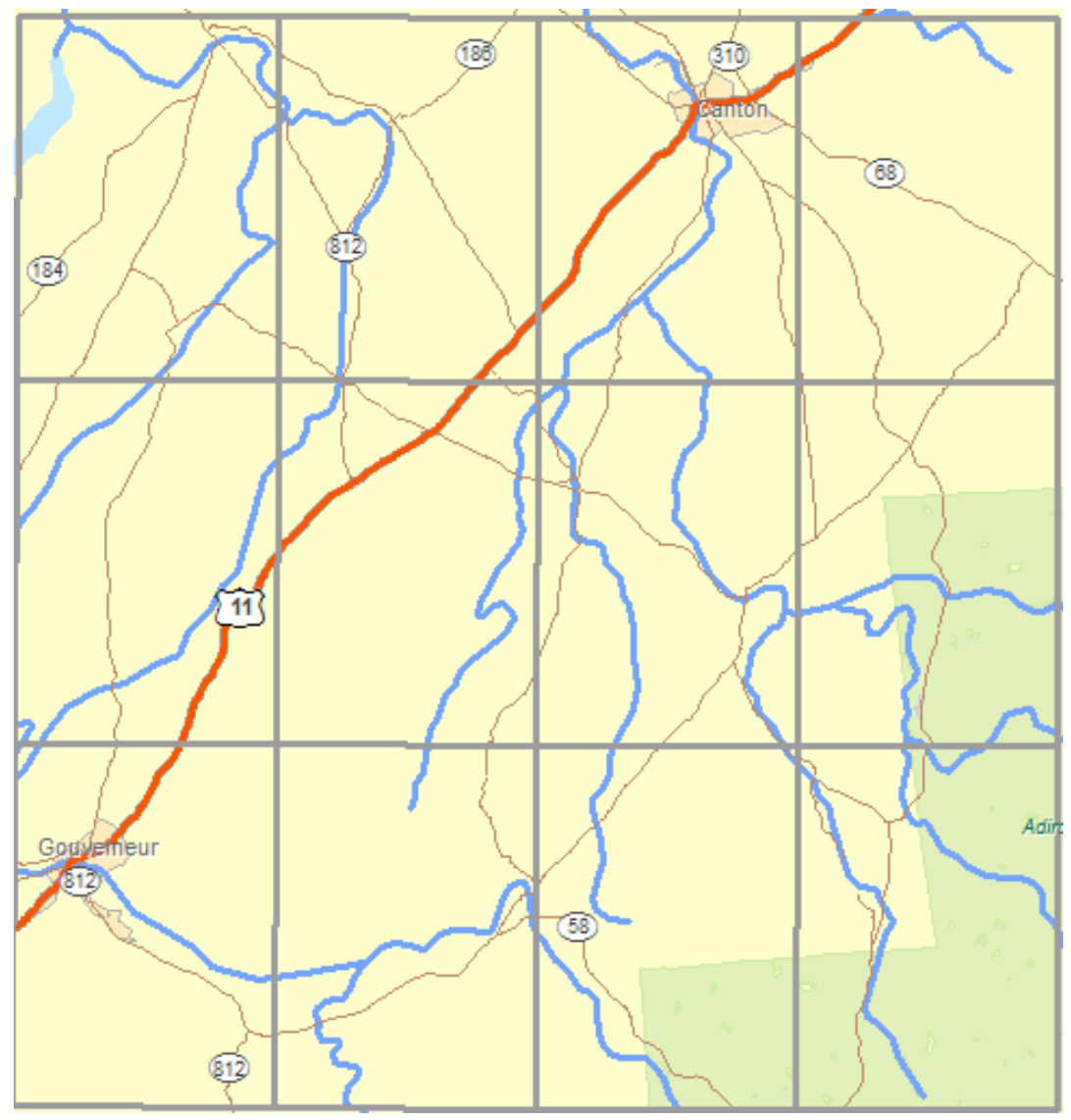

Figure 6.1 Original GIS data in ArcMap. 
To create an initial model, a feature dataset was created to store all of the versioned feature classes, using Create Feature Dataset from the ArcAEM v. 3.0 toolbar. The default spatial reference for this tool is that of the ArcMap data frame, but a spatial reference dialogue appears (Figure 6.2) to allow the user to change this setting. The user may then import or browse to a spatial reference. In this case, the data frame's spatial reference, NAD_1983_UTMZone_18N, was used to create the feature dataset.

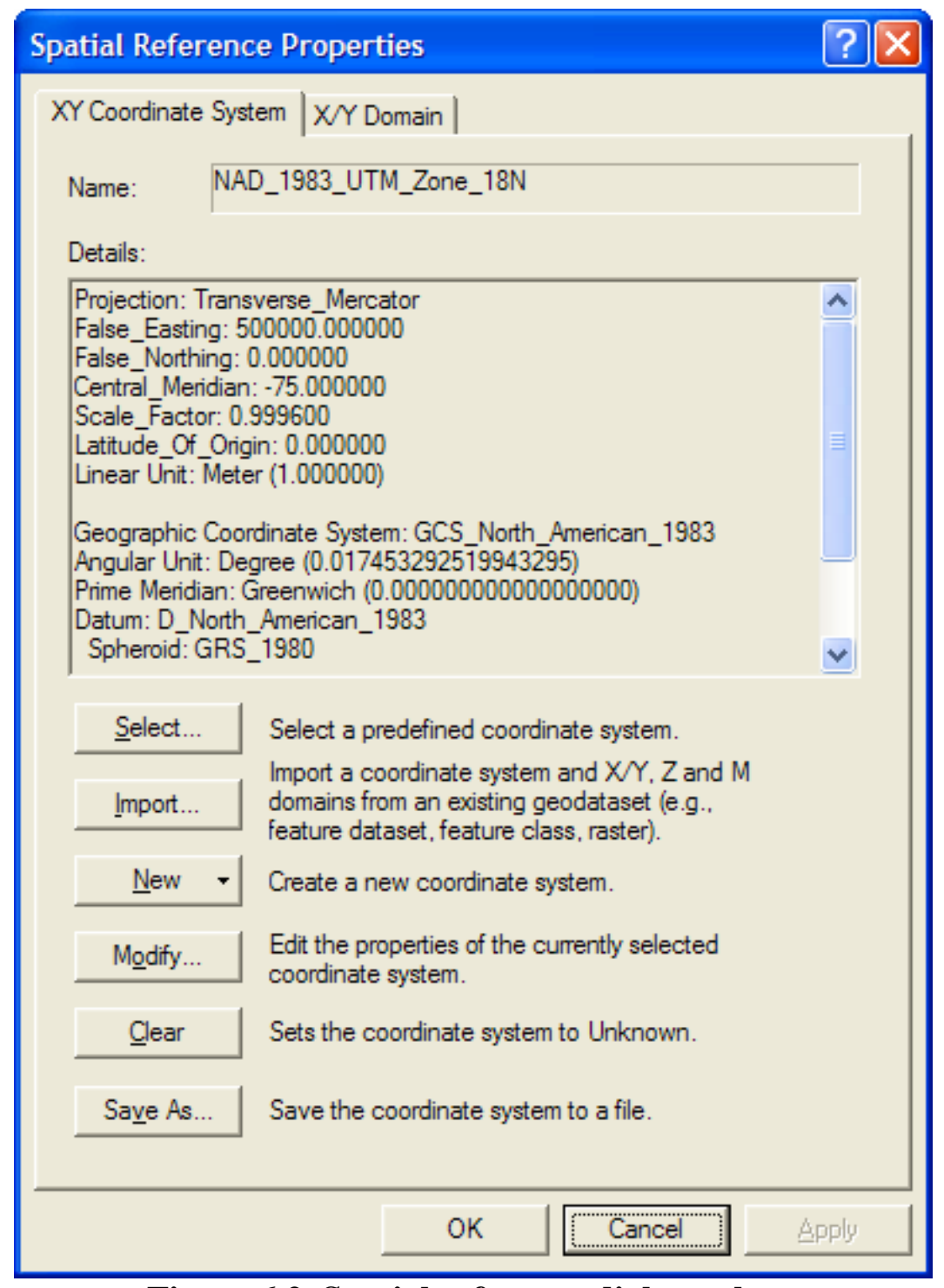

Figure 6.2. Spatial reference dialogue box.

Feature classes were created to represent the groundwater features in that area inside the feature dataset. The feature classes inherited the spatial reference from the feature dataset. The features created for this model included rivers, wells, a recharge zone, and inhomogenieties (regions of significant difference in porosity). Using the ESRI Editing toolbar, these feature classes were populated with groundwater features to create the first model (Figure 6.3). 


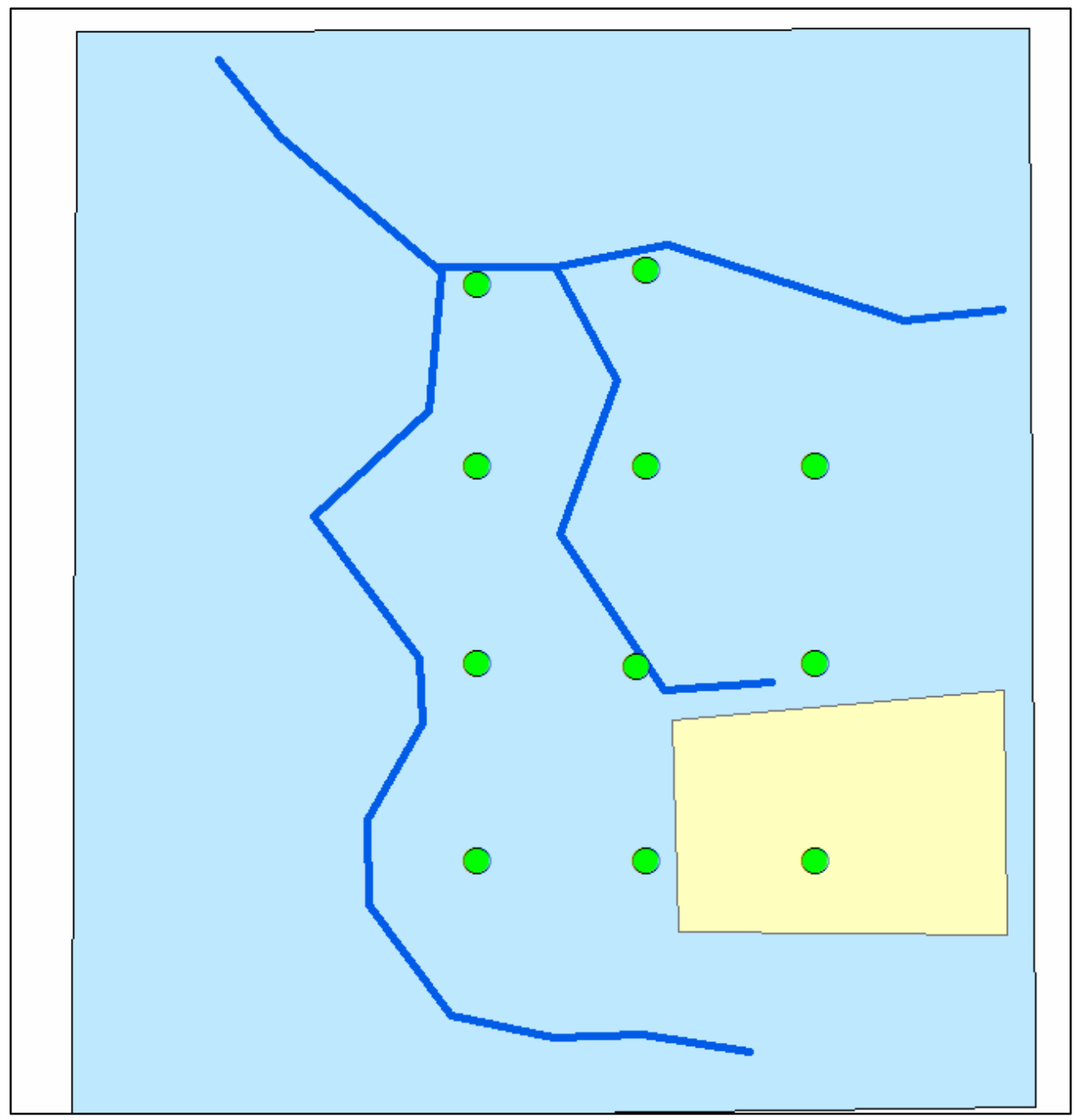

Figure 6.3. First groundwater model.

\subsection{Registering the Feature Dataset}

Once the original model features are created, the feature dataset was registered as versioned using the Modeling submenu.

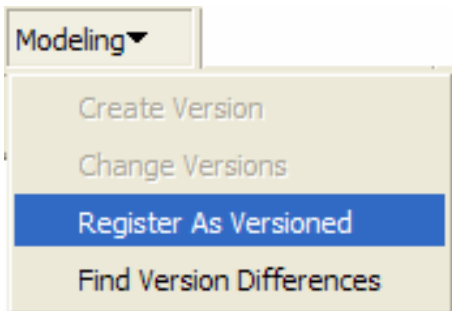

Figure 6.4. ArcAEM v. 3.0 Modeling menu. 
Registering the data as versioned caused the ArcGIS software to create the system tables and delta tables in the RDBMS. Figure 6.5 illustrates the addition of the four system tables in Microsoft SQL Server 2005: Mvtables_Modified, State_Lineages, States, and Versions.

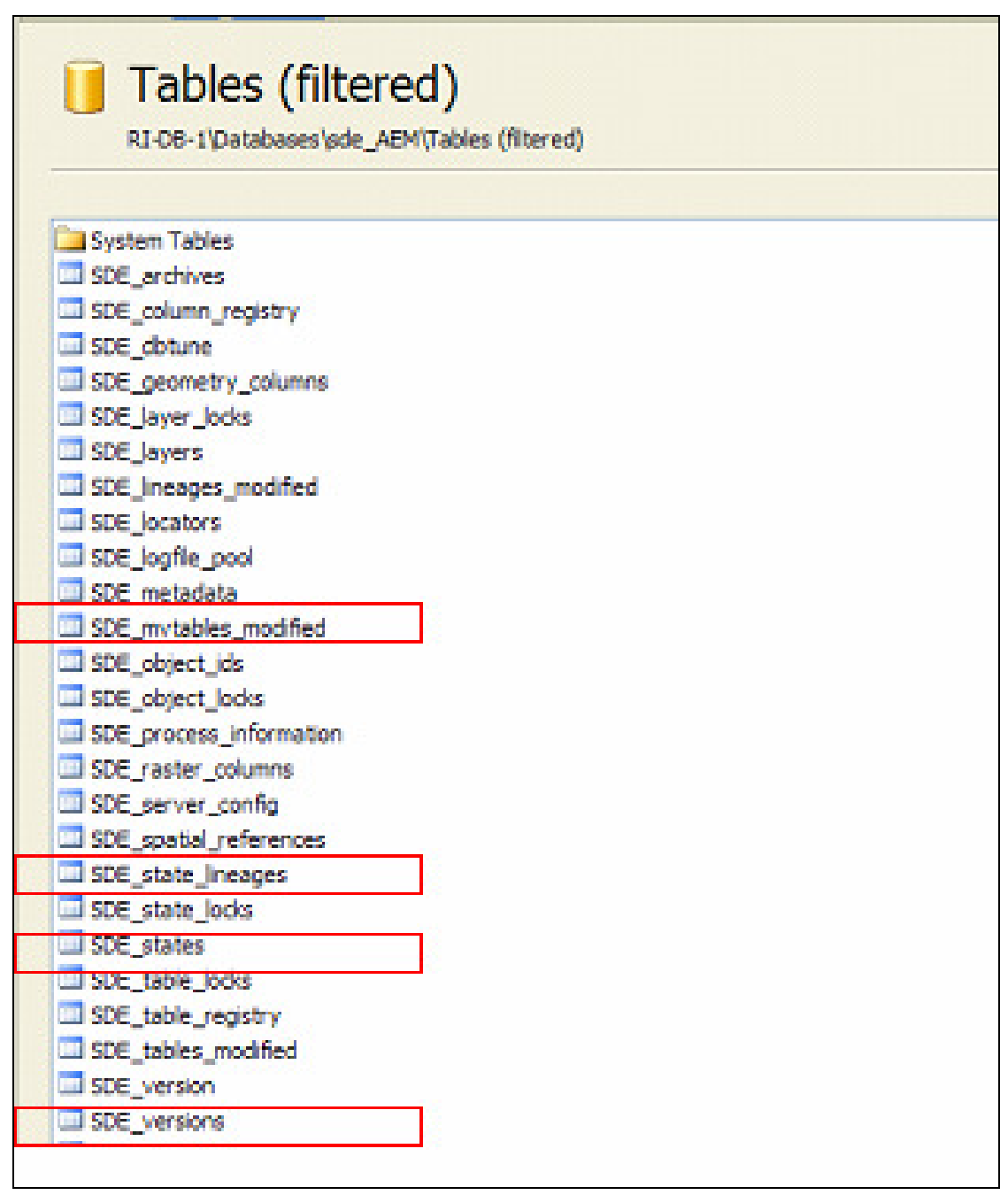

Figure 6.5. Tables Added to ArcMap for Versioning.

\subsection{Creating a New Model}

New models were created from the default version using the Create Versions submenu. From this menu, an interface appears that allows the user to choose a new name for the version, a description if necessary, and permission type (Figure 6.6). The user may also request to switch to the display of that new version. 


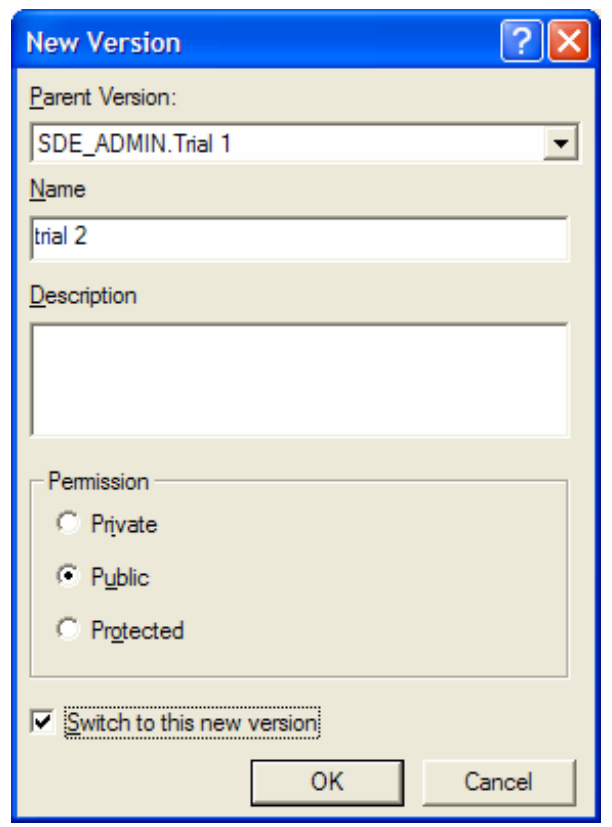

Figure 6.6. Create New Version Dialogue.

Adding a new version creates a new row in the Versions table (Figure 6.7). The inserted record contains the version name, owner, status, current state ID, and the parent version information.

\begin{tabular}{|c|c|c|c|c|c|c|c|c|c|}
\hline \multicolumn{6}{|c|}{ RI-DB-1.master - SQLQuery1.sql Table - sde.SDE_versions } & \multicolumn{4}{|l|}{ Summary } \\
\hline & name & owner & version_id & status & state_id & parent_name & parent_owner & parent_version_id & creation_time \\
\hline \multirow[t]{4}{*}{ 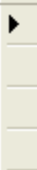 } & DEFAULT & sde & 1 & 1 & 16 & : MULL & NULL & NULL & 7/20/2007 9:21:13 AM \\
\hline & Trial 1 & SDE_ADMIN & 38 & 0 & 103 & DEFAULT & SDE & 1 & $10 / 12 / 20078: 08: 30 \ldots$ \\
\hline & Trial 2 & SDE_ADMIN & 39 & 1 & 97 & DEFAULT & SDE & 1 & $10 / 13 / 20079: 13: 57 \ldots$ \\
\hline & trial 3 & SDE_ADMIN & 41 & 1 & 95 & Trial 1 & SDE_ADMIN & 38 & $10 / 18 / 20078: 06: 41 \ldots$ \\
\hline *⿻丷木 & MULL & MULL & MULL & MULL & MULL & MULL & NULL & MULL & NULL \\
\hline
\end{tabular}

Figure 6.7. New Versions added to versions table.

After the new model is created, changes can be made using the ESRI Editing toolbar. For this particular model, wells were added to the new model and river geometry was changed. The changes made to this and any subsequent version are recorded in the delta tables. Adding a head observation creates a new entry into the Adds table, while a removal is recorded in the Deletes table. The Adds table in the RDBMS references the new feature ID, the attributes, and the state ID (Figure 6.8). Within a single edit session, the state IDs for all subsequent additions to the geodatabase remain the same. 


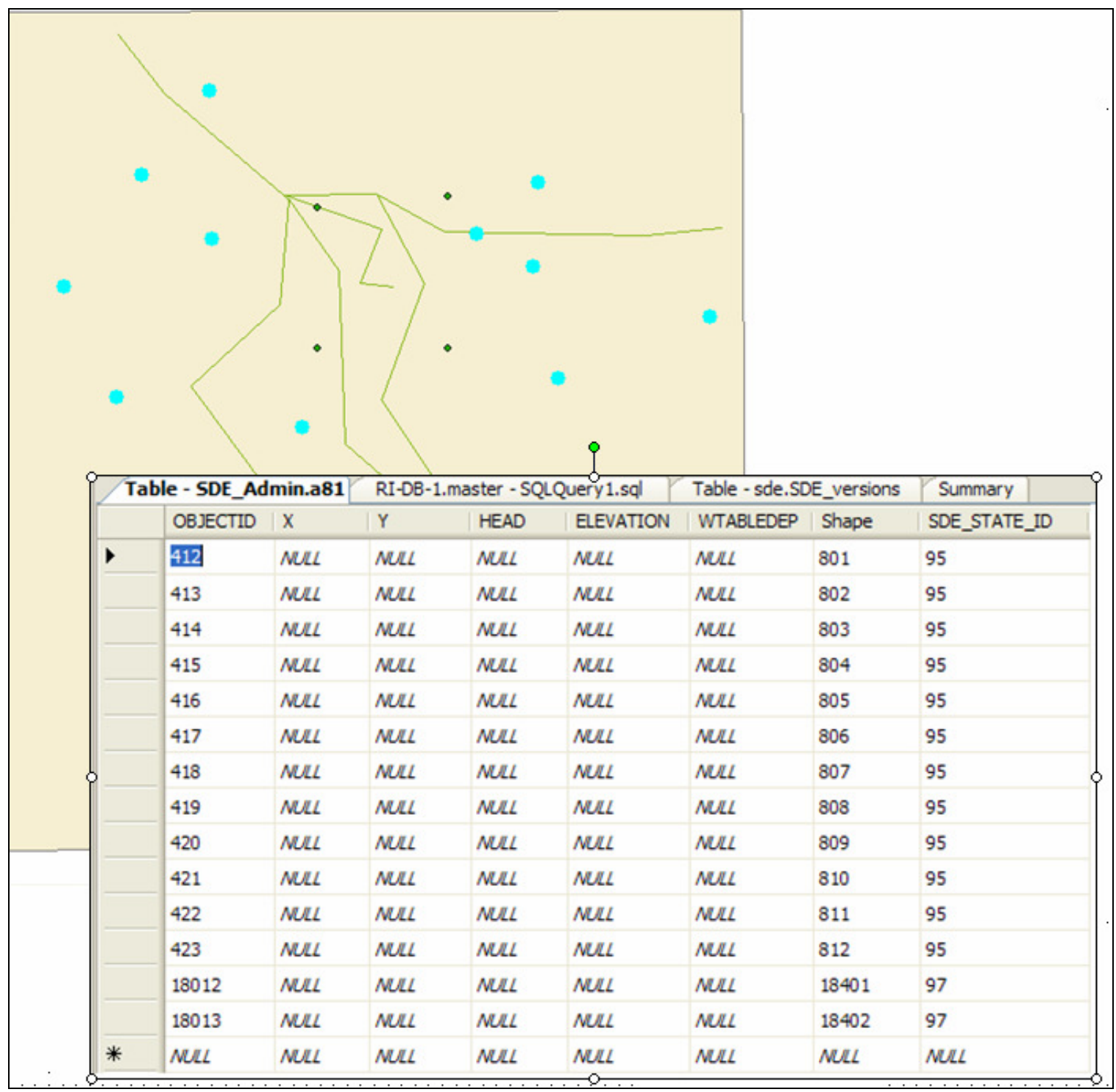

Figure 6.8 Added features in ArcMap and Adds table.

Removing a head observation creates a record in the Deletes table referencing the state ID, and the object ID of the deleted feature (Figure 6.9). 


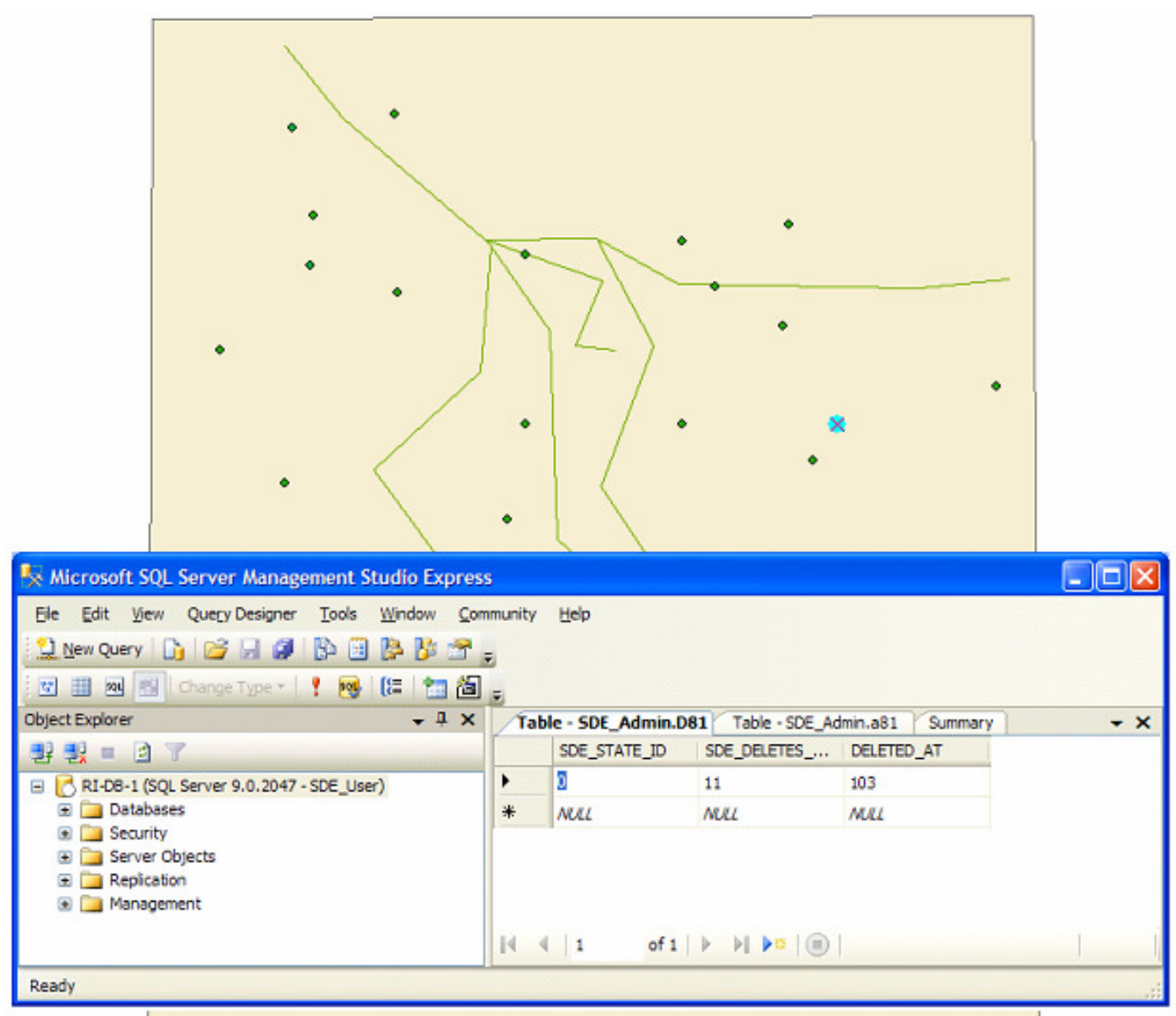

Figure 6.9. Feature removal in ArcMap and Deletes table.

Changing the feature geometry for a river created two records: one in the Adds table and one in the Deletes table (Figure 6.10). The old river geometry was first removed, and the new river geometry was then added. Changes can be made to both feature attributes and feature geometry. When the new river geometry was added, a new shape ID for the river geometry was added as well. For attribute changes, the new attributes were recorded within the add record itself and the OID was maintained. 


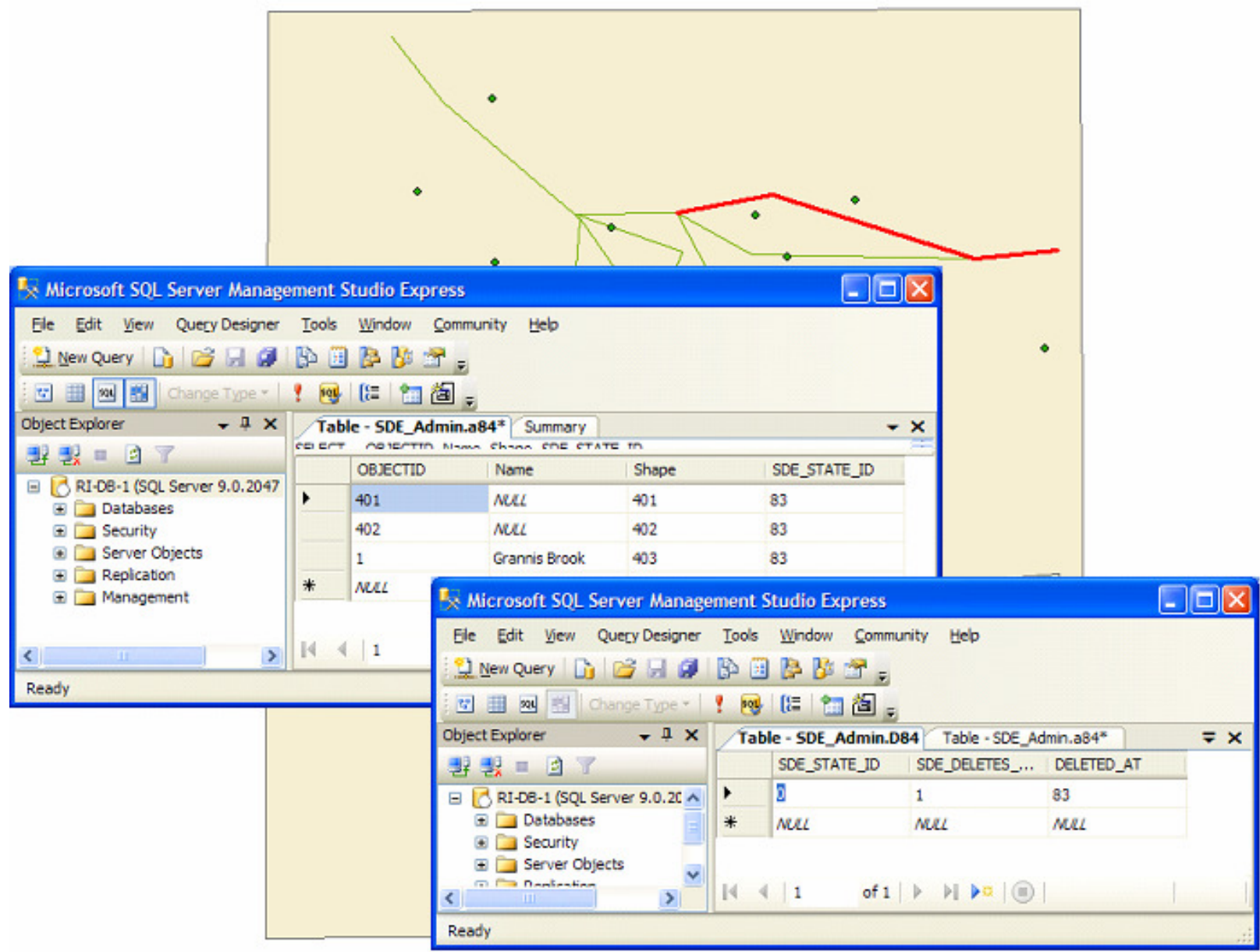

Figure 6.10. Changed feature in ArcMap, Adds table, and Deletes table.

After modeling changes were made, a list of all changed attributes between two versions was created. The version report submenu provides a user interface that shows the list of changes, provided the names of the compared versions and the feature datasets one wishes to compare (Figure 6.10). 


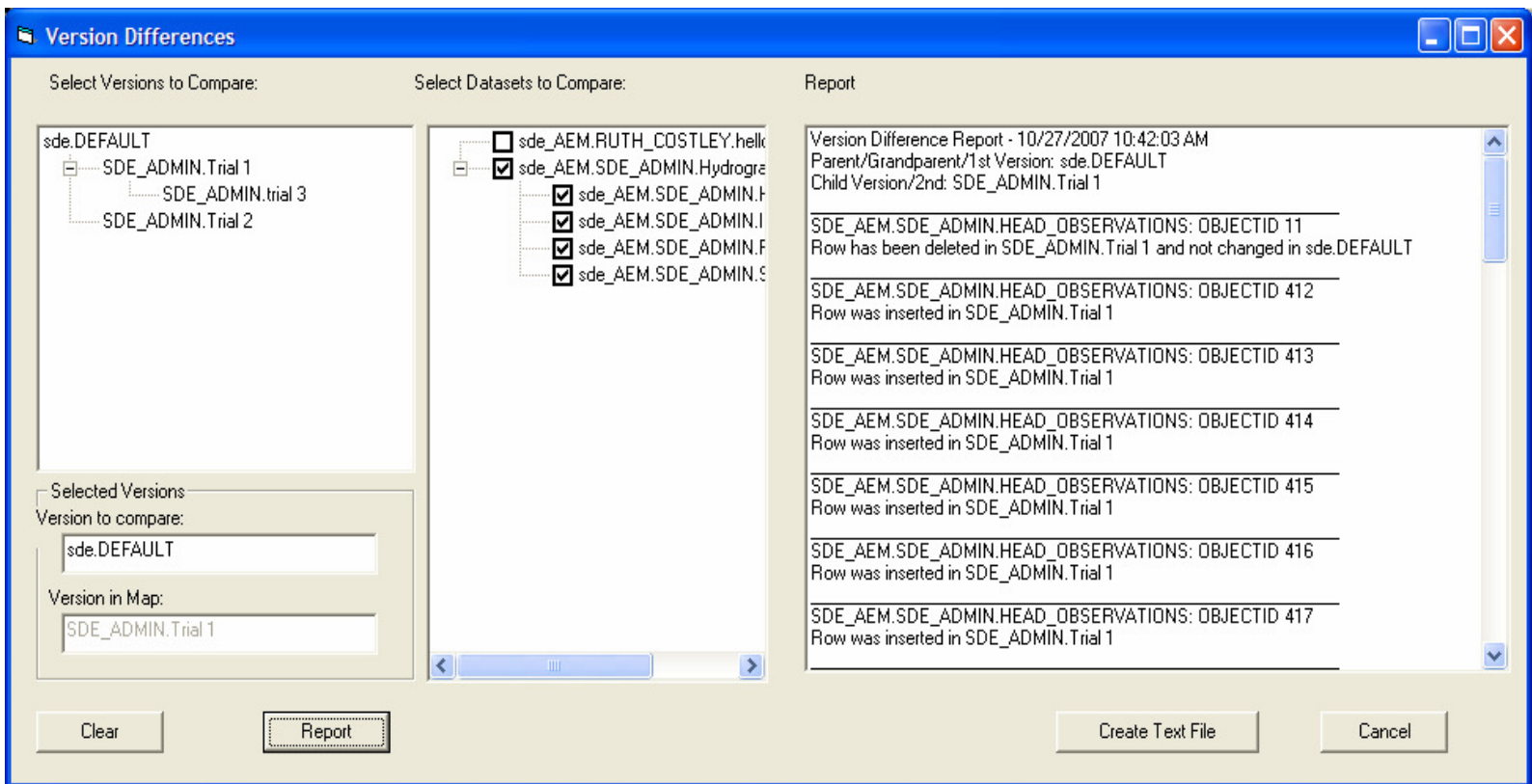

Figure 6.11. Find Version Differences dialogue box.

The list is a dynamic list that allowed features to interact with the map through the change report. The user may zoom to map features and also see geometry changes on the map (Figure 6.12).

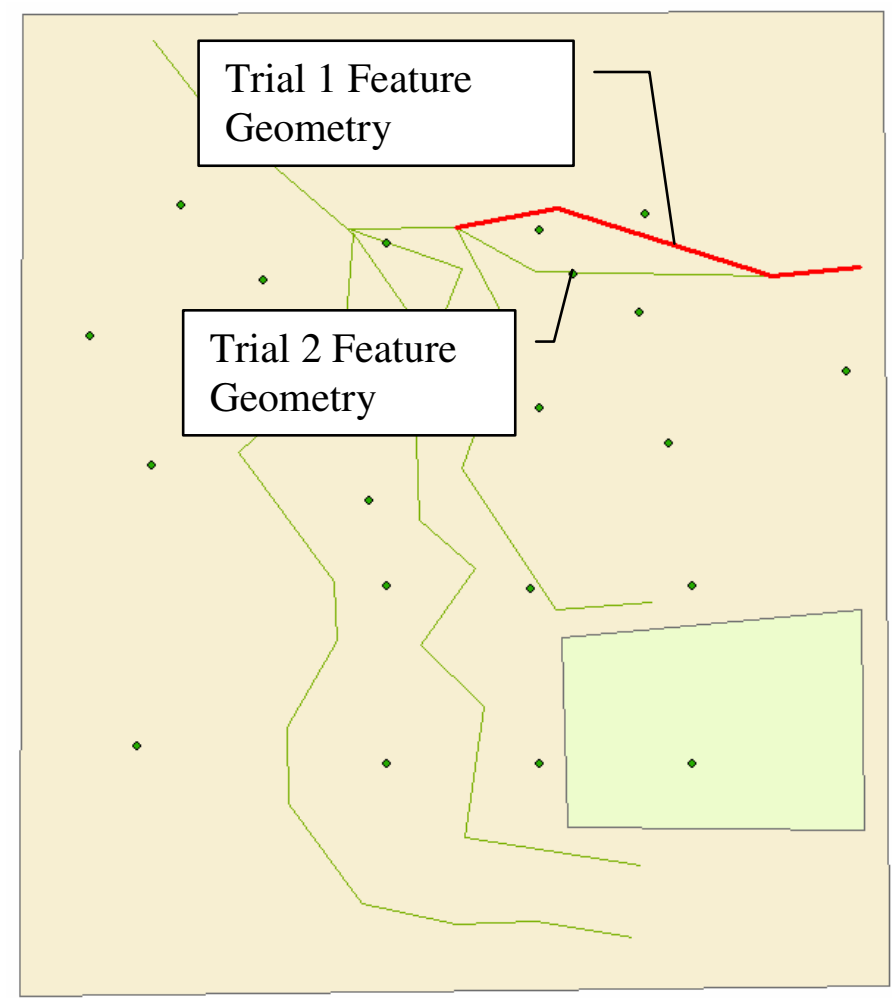

Figure 6.12. Geometry change between versions. 


\subsection{Test Summary}

The workflow described in this section created multiple models of a given dataset.

However, there is a potential issue with regard to degraded system performance as the number of changes and separate versions increases. While the effect was not noted in the simple models used in testing phase, the point where degraded performance might occur is not known.

Individual models can be computed within the SPLIT solver to produce surfaces and particle tracking grids. Different versions of the data will produce different results for these grids, since different analytic elements will be used in the input parameters. The resulting changes could be minute or drastic depending on the changes within the models themselves. Currently no mechanism exists to differentiate between two grids other than examining the difference. 


\section{Summary and Future Work}

With this enhancement to ArcAEM, the scientist is allowed more freedom to create multiple models and build on previous models without having to start from scratch or create redundant data. This freedom to create models improves the scientific process for modeling with ArcAEM. The user is also able to keep track of the differences between each model which will also help the scientist record their work. A versioning workflow could be incorporated into other modeling applications.

The ArcAEM toolbar is an ongoing research application that will be continually improved over time. The new buttons created are capable of easily implementing a versioning system, but more functionality could be added to improve the analysis within versions and to further improve processing time. A few more tools could be implemented, which are explained in the next sections.

\subsection{Copying Features}

One future enhancement to versioning AEM models would be the ability to copy features between versions, while maintaining the features versioned state. Features copied between the geodatabase would be given a new object identification number, a new record of itself. To truly version the database, the copied features would be a representation of the original and would maintain the same object identification number. This ability is currently limited by the ArcSDE technology, which does not track the existence of an object.

Research by Hornsby and Egenhofer (2000) offers one approach to track identity for spatial features. They describe four primitives of identity-based change: object from non-existence to existence; object continues to exist; object from existence to nonexistence; and object continues to not exist (Figure 7.1).

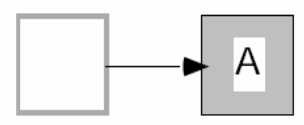

(a)

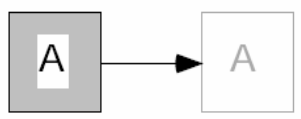

(b)

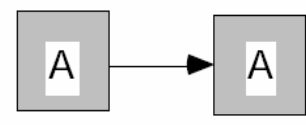

(c)

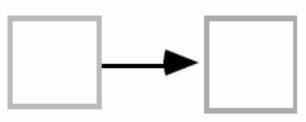

(d)

Figure 7.1 Four Primitives of Identity. a) Feature is created. b) Feature removed. c) Feature continues to exist. d) Feature continues to not exist. Note. From Hornsby and Egenhofer, 1998.

To copy features from one version to another, the feature in the new version must change from non-existence in that line to existence or be created again. The limiting factor is that an object can only be created once within the geodatabase. For copying, history should be allowed in the design, so that a feature could have non-existence with history, whereby although the feature does not exist in it may exist somewhere else. Furthermore, a feature could have non-existence with premonition, whereby the creator of the feature knew at some point it would exist. Figure 7.2 further describes this process. In diagram a, the figure is created in part of the version tree. In diagram $b$, the version is then copied to its cousin. For the feature to exist in figure b, that line of the tree must 
realize that the feature has history and therefore apply it. In ArcSDE, this would require an additional change table that would essentially be the existence of an object table. In this way, a feature would not have to be permanently removed from the version, just checked into existence.
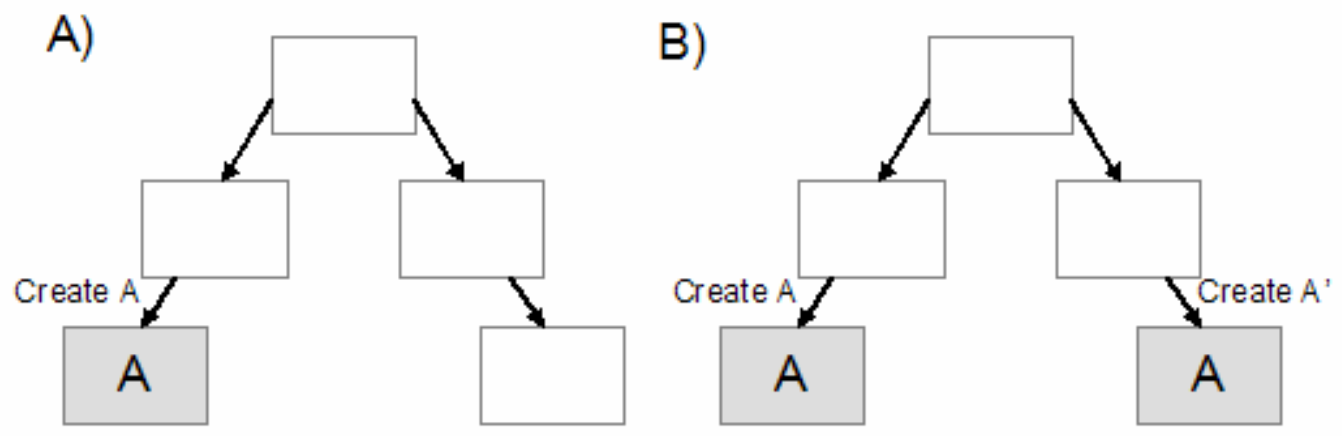

Figure 7.2 Primatives of identity within a versioned tree. a) Features is created. b) Feature is applied to another branch of the tree.

\subsection{Change Accumulation}

Incorporating the version differences tool is a useful tactic to improve subsequent models. This tool could be further developed to provide change accumulation. A complete list of changes could be created for the entire set of models within the geodatabase with the total amount of change between the default and any child version. In this case, the total number of changed records and their change type would be recorded within each model from parent to child and further printed as a list. The modeler could also quantify the differences in an accumulated change, defined by totaling each subsequent model from its parent and totaling it over the entire lineage.

\subsection{Versioning Surfaces}

Versioning the output surfaces could decrease processing time for each model. The groundwater solver uses a text file for its calculations. Each given element contained in the geodatabase creates a line of text to be submitted to this solver. The act of versioning the output surfaces would first be to keep the groundwater solver from solving for the same features twice. In this case, a marker could be created within the text file to indicate a new attribute value and have only that attribute value calculated by SPLIT.

The next step to this process would then version the input into spatial analyst that further interpolates the surface. To produce these surfaces, the groundwater solver produces a series of values in a grid like pattern across the plane of study and interpolates these points. If few changes were made to the groundwater solver, then many of the values would be the same. These same values could be flagged and areas that are not influenced by new values would not need to be interpolated. Change areas could be reported by dividing the entire study area into a square grid and highlighting those grids that have been changed between models. 


\subsection{Graphical User Interface Modification}

The current graphical user interface in the Find Version Differences dialogue box could be improved to better support users of ArcGIS products. The versioning tree produced within Find Version Differences dialogue box is a standard control within VB 6 that creates a tree. A new user interface could be created from scratch to enhance the tree. The tree could also highlight the current version that is being viewed by the user. This change could help users view their models and enhance their versioning workflow. 



\section{References}

Arctur, D. \& Zeiler, M. Eds. (2004). Designing geodatabases: case studies in GIS data modeling. Redlands, CA: ESRI Press.

Bernard, E. A., Steward, D. R., \& Le Grand, P. (2005). A geodatabase for groundwater modeling in MLAEM and MODFLOW. ESRI International User Conference, San Diego, CA. Retrieved January 29, 2007, from http://training.esri.com/campus/library/index.cfm.

Burrows, W. E., \& Langford, J.D. (2000). Programming business applications with Microsoft Visual Basic 6.0. Boston, MA: Irwin McGraw-Hill.

Couclelis, H. (1992). People manipulate objects (but cultivate fields): beyond the raster vector debate in GIS. Lecture Notes in Computer Science, 639, 65-77.

Cellary, W., \& Jomier, G. (1990). Consistency of versions in object-created databases. Proceedings of the $16^{\text {th }}$ Very Large Database Conference. Brisbane Australia, 432-441. Retrieved January 29, 2007, from http://sigmond.org/vldb/conf/1990/P432.PDF.

Csoma, R. (2001).The analytic element for groundwater flow modeling. Periodica Polytechnica, 45 (1) 43-62. Retrieved January 29, 2007 from http://www.pp.bme.hu/ci/2001_1/pdf.

Douglas, D., \& Peucker, T. (1973). Algorithms for the reduction of the number of points required to represent a digitized line or its caricature. The Canadian Cartographer, 10(2), 112-122.

ESRI. (2000). Developing applications with ArcInfo: an overview of ArcObjects. Redlands, CA: ESRI. Retrieved October 29, 2007 from http://support.esri.com.

ESRI. (2004). Versioning: an ESRI technical paper. Redlands, CA: ESRI. Retrieved July 1, 2007, from http://support.esri.com.

ESRI. (2006). Geodatabase archiving: Introduction to concepts and capabilities. Redlands, CA: ESRI. Retrieved July 20, 2007 from http://suppport.esri.com.

Fredrick, K.C., Becker, M.W., Matott, L.S., Daw, A., \& Flewelling, D. M. (2007). Development of a numerical groundwater flow model using SRTM elevations. Hydrogeology Journal, 15(1), 171-181.

Hornsby, K. \& Egenhofer, M. (1998). Identity-based change operations for composite objects. $8^{\text {th }}$ International Symposium on Spatial Data Handling. Vancouver, Canada. 202-213. Retrieved October 24, 2007 from http://www.spatial.maine.edu.

Hornsby, K. \& Egenhofer, M. (2000). Identity-based change: a foundation for spatiotemporal knowledge representation. International Journal of Geographic Information Science, 14(3). 207-224. Retrieved October 24, 2007 from http://www.ingenta.com.

Hunt, R. J. (2006). Groundwater modeling applications using the analytic element method. GroundWater, 44(1). 5-14. 
Maidment, D. R. (Ed.) (2002). Arc Hydro: GIS for water resources. Redlands, CA: ESRI Press.

Rabideau, A. J., Craig, J.R., Silavisesrith, W., Fredrick, K., Flewelling, D. M., \& Jankovic, I., et al. (2007). Analytic-element modeling of supraregional groundwater flow: concepts and tools for automated model configuration. Journal of Hydrologic Engineering, 12(1), 83-96.

Schwartz, F., \& Zhang, H. (2003). Fundamentals of groundwater. New York, NY: John Wiley \& Sons, Inc.

Silavisesrith, W. \& Matott, L. S. (2005). ArcAEM: GIS-based application for analytic element groundwater modeling; documentation and user's guide. Unpublished manuscript. Retrieved February 15, 2007 from http://www.groundwater.buffalo.edu/software/ArcAEM/ArcAEMMain.html.

Smith, Z. (1989) Groundwater in the west. San Diego, CA: Academic Press, Inc.

Steward, D. R. \& Bernard, E. A. (2006). The synergistic powers of AEM and GIS geodatabase models in water resources studies. Ground Water, 44(1). 56-61.

Strassberg, G. \& Maidment, R. (2004). Arc Hydro groundwater data model. AWRA Spring Speciality Conference. Nashville, TN. Retreived August 19, 2007, from https://webspace.utexas.edu/gstras/MyWebsite/publications/GilStrassberg_44a15 8a_1641.pdf.

Strack, O.D. (1989). Groundwater mechanics. Englewood Cliffs, NJ: Prentice Hall.

Talens, G., \& Oussaiah, C. (1993). Versions of simple and composite objects. Proceedings of the $19^{\text {th }}$ Very Large Database Conference. Dublin Ireland, 62-72. Retrieved January 29, 2007, from http://www.informatik.unitrier.de/ ley/db/conf/vldb/TalensOC93.html.

Tryfona, N., \& Egenhofer, M. J. (1997). Consistency among parts and aggregates: A computational model. [Electronic Version]. Transactions in GIS, 1(3), 189-206.

USGS What is hydrology and what do hydrologists do? (2005). Accessed on February 10, 2007. Retrieved February 5, 2007 from http://ga.water.usgs.gov/edu/hydrology.html.

Vangenot, C., Parent, C., \& Spaccapietra, S. (July, 2002 ). Modeling and manipulating multiple respresentations of spatial data. Proceedings of the Symposium on Geospatial Theory. Ottawa, Canada. Retrieved January 29, 2007, from http://lbdsun.epfl.ch/e/publications_new/articles.pdf/SDH_Vangenot.pdf.

Wang, H. \& Anderson, M. (1982). Introduction to groundwater modeling: Finite Difference and Finite Element methods. San Francisco, CA: W. H. Freeman and Company. 


\section{Appendix A - ArcAEM Tutorial 7}

A tutorial was created for use by the University of Buffalo, and the general line. This followed tutorials already created for ArcAEM.

\section{ArcAEM Modeling Tutorial \#7: Utilizing the Modeling Submenu with ArcSDE}

The following tutorial is designed to introduce a modeling workflow using the Modeling Submenu in ArcAEM. By the end of this tutorial, the user should be able to:

- Register an ArcSDE feature dataset or class as versioned

- Create new versions

- Change versions

- Create a list of version differences

For this tutorial, the user is assumed to have an ArcSDE license with the accompanying relational database management system. The user should have read/write access to a geodatabase instance.

\section{Step 1: Create First Model}

The first model should consist of all the analytical element feature classes needed for the execution of SPLIT. All of these feature classes will furthermore be contained within the Hydrography feature dataset in the ArcSDE geodatabase instance. Use the following steps to build your first model.

1. Click on the Create Feature dataset button ( $)$. A browse dialogue will appear. Browse to your ArcSDE geodatabase instance, and specify a name for your new feature dataset. After you click Save, a spatial reference dialogue box will 
appear. The spatial reference referenced by this dialogue is that of the data frame. Change as necessary.

2. Create feature classes within the feature dataset that will aide with modeling application. To create a specific model, see Tutorial 2 Step 3.

\section{Step 2: Register the Feature Dataset as Versioned}

Registering the feature dataset will register all the included feature classes at the time of registration. This process will allow you to create new models or versions of the data.

First click on the source tab at the bottom of the Table of Contents (Figure 1). Highlight the Hydrography feature dataset (Figure 1). From the Modeling menu, click the Register as Versioned submenu. A message box will appear when the process is complete. 


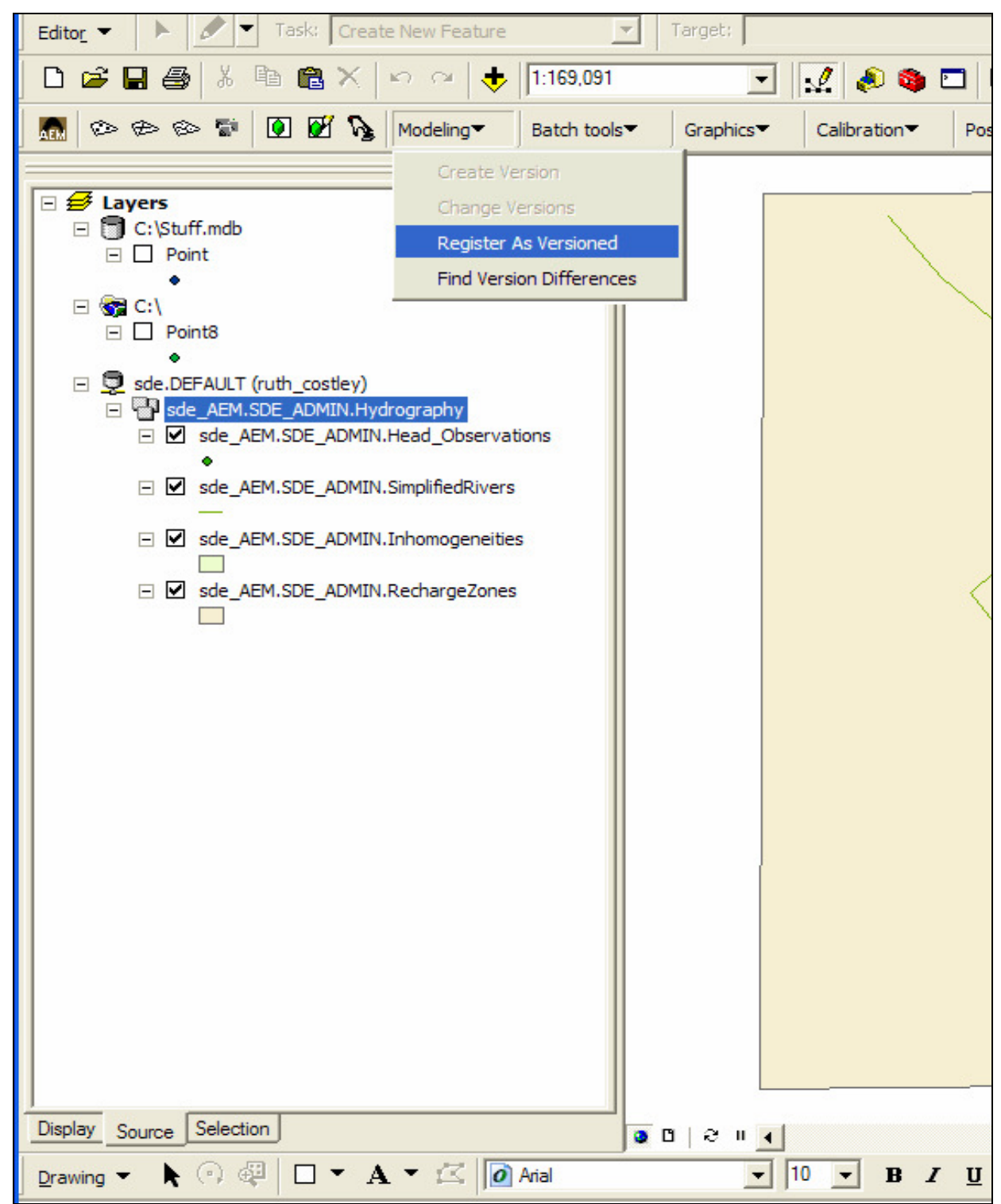

Figure 1: Registering a Feature Dataset as Versioned.

\section{Step 3: Create a New Model}

New models can be created by adding features, removing features, changing feature geometry, and changing feature attributes to a new version of the previous version. To create a new model, you will first have to create a pointer to that new model; this is referred to as a version.

With the source tab selected, highlight the geodatabase instance. From the Modeling menu, click the Create Version submenu. A form 
will display (Figure 2). For name, type first trial. For permission, choose public. Click the check box next to Switch to this new version. Use the example below to compare your answers. Once the form has been filed, click OK. The new version of the geodatabase should appear on screen.

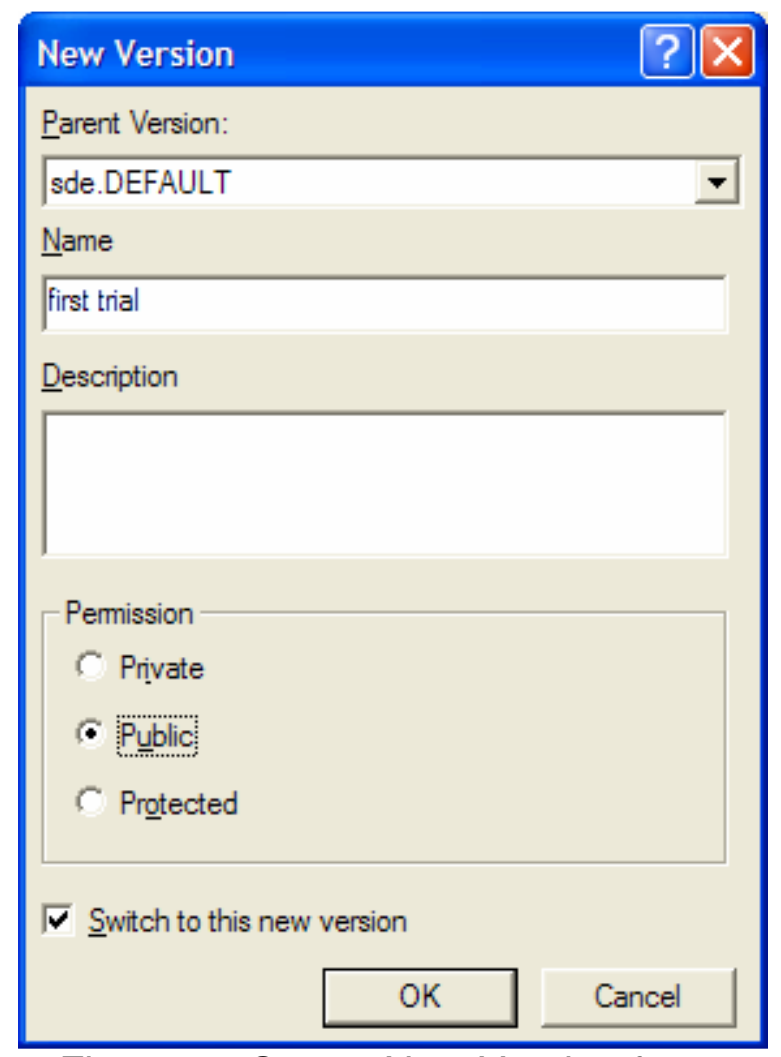

Figure 2: Create New Version form.

With the new version, create your new model by adding features, removing features, changing feature attributes, and changing feature geometry. The changes can be made using the Editing toolbar.

\section{Step 4: Change Versions}

During your ArcMap session, you may want to display a previously made model on screen. For this you will need to change the connection property of the geodatabase to that of the other version.

From the Modeling Menu, choose the Change Version submenu. A form like the one in figure 2 should display. Leave the default version 
type as transactional, highlight the version you wish to change to, and press ok.

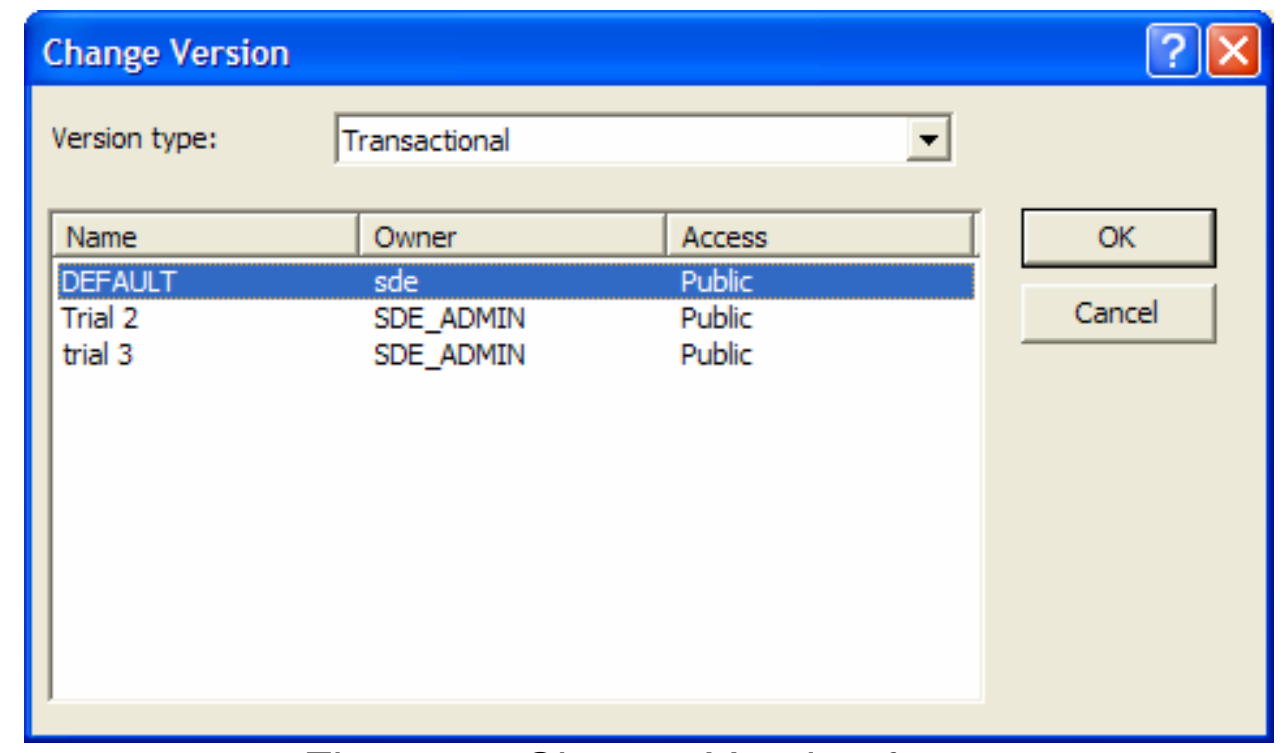

Figure 3: Change Version form.

The previous created model should now be displayed on screen. From this version, you may create new models from it using the Create Version button or change the version by adding, removing, and changing features. Just remember that once you change a version you are changing it permanently, if you want to leave it as a marker, just create a new version from it.

\section{Step 5: Generate a Version Differences Report}

A version differences report will give a list of added, removed, or changed feature classes.

From the Modeling menu, choose the Find Version Differences submenu. The current version of the database is auto filled in the Version in Map box (Figure 4). To select a version to compare, right click on the version and choose version to compare from the context menu. Select a versioned dataset to compare to. Finally click the Generate Report button. A list of all the changed features should appear in the Report list. 


\section{This is an interactive list. Highlight and right click geometry change to view the other geometry on screen.}

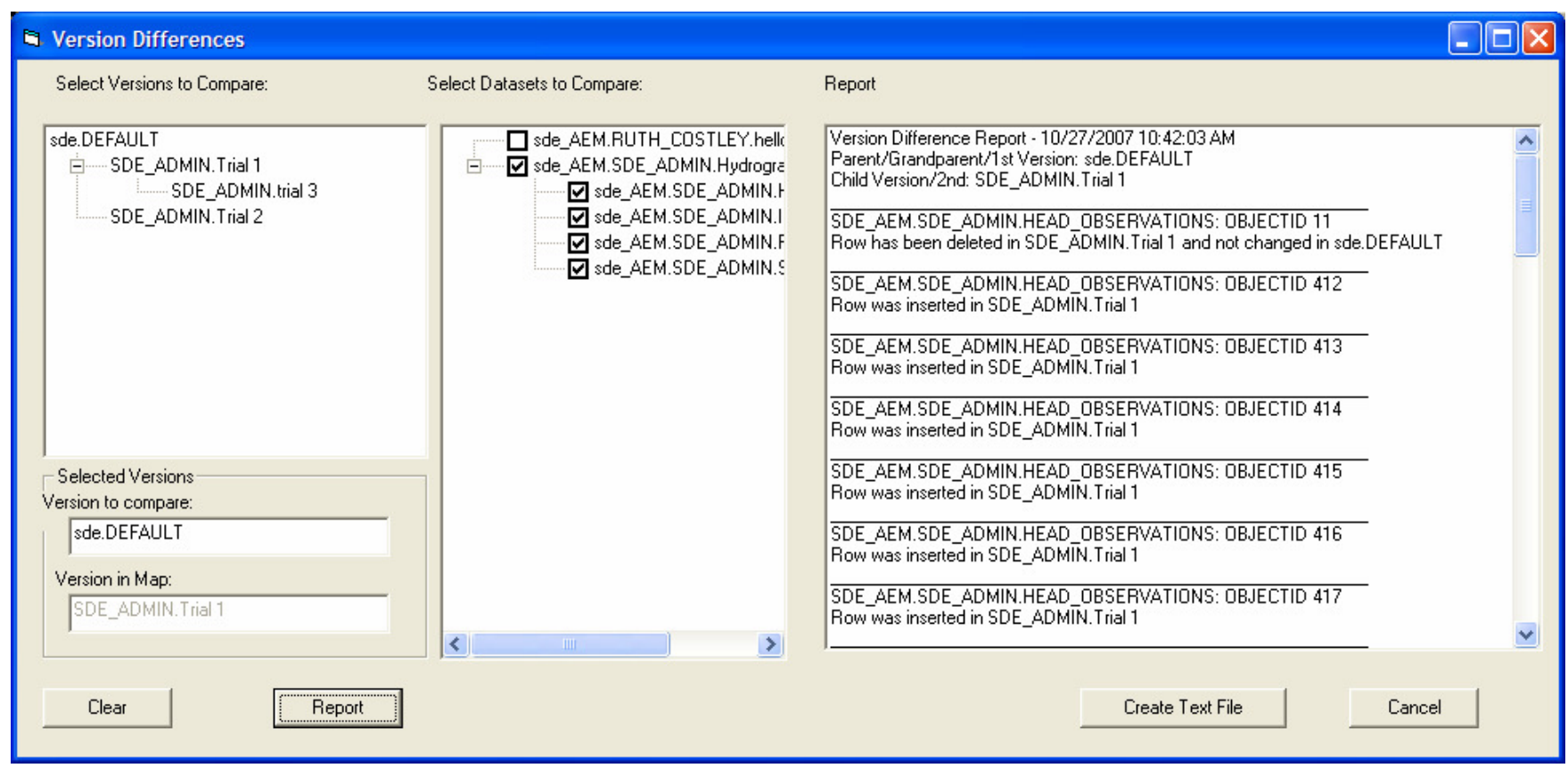

Figure 4: Version Differences Form. 


\section{Appendix B - Create Feature Dataset}

This is the code that comprises the CreateFeatureDataset.cls. This code will create the Create Feature Dataset button on the Modeling submenu.

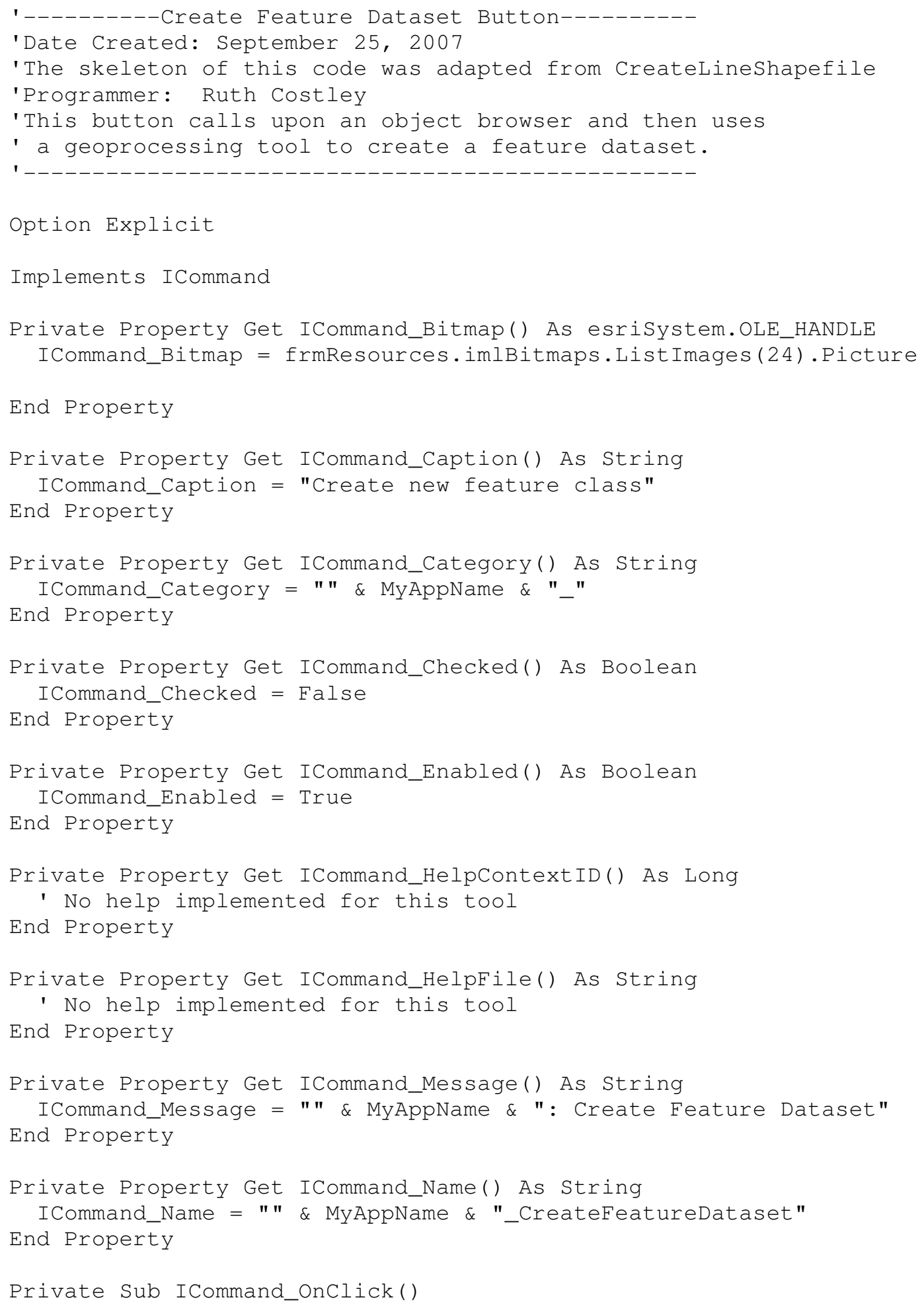




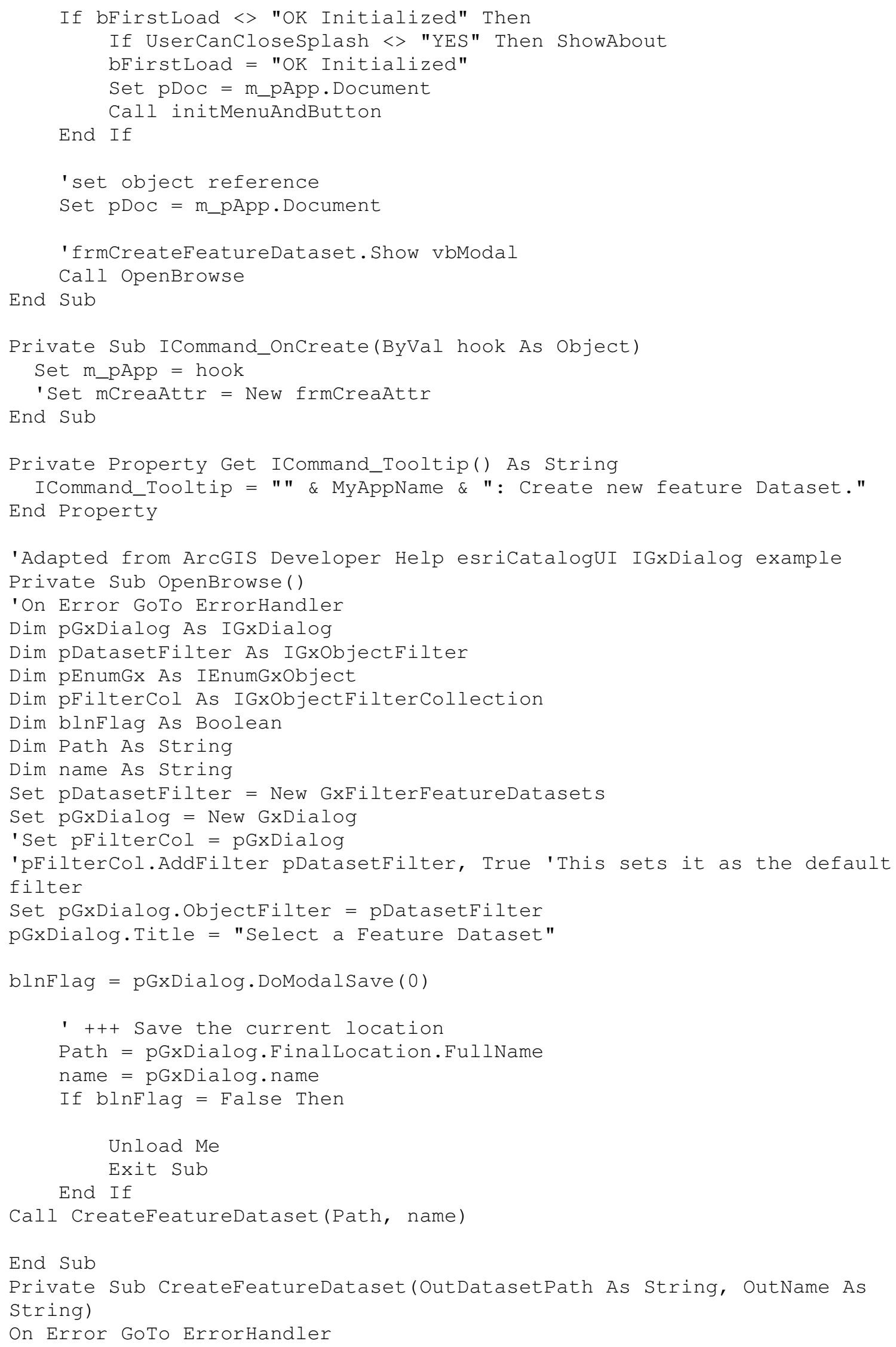




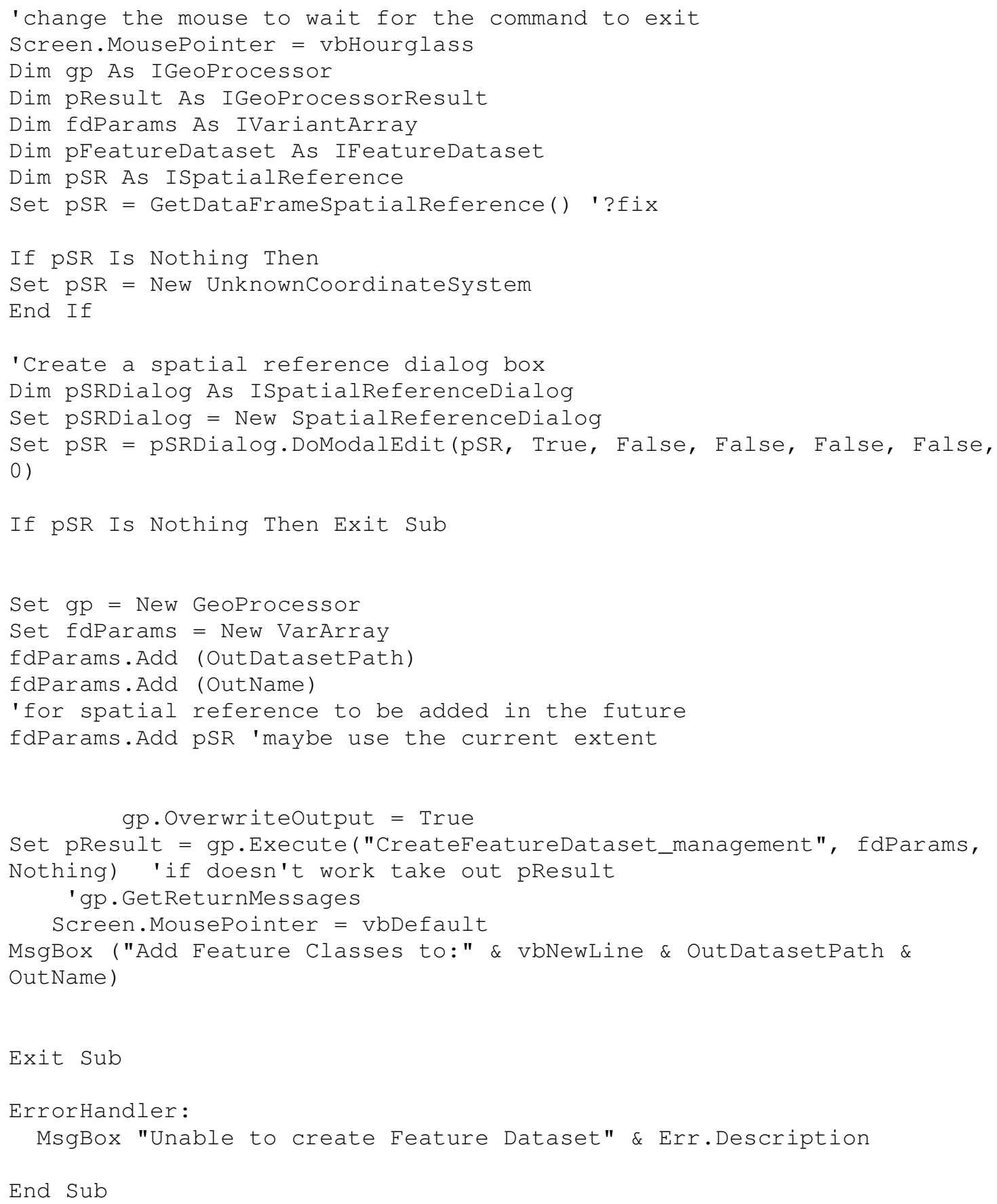





\section{Appendix C - Register As Versioned in VB 6.0}

This is the code in registeredVersioned.cls. This code will operate the Register as Versioned submenu on the Modeling toolbar.

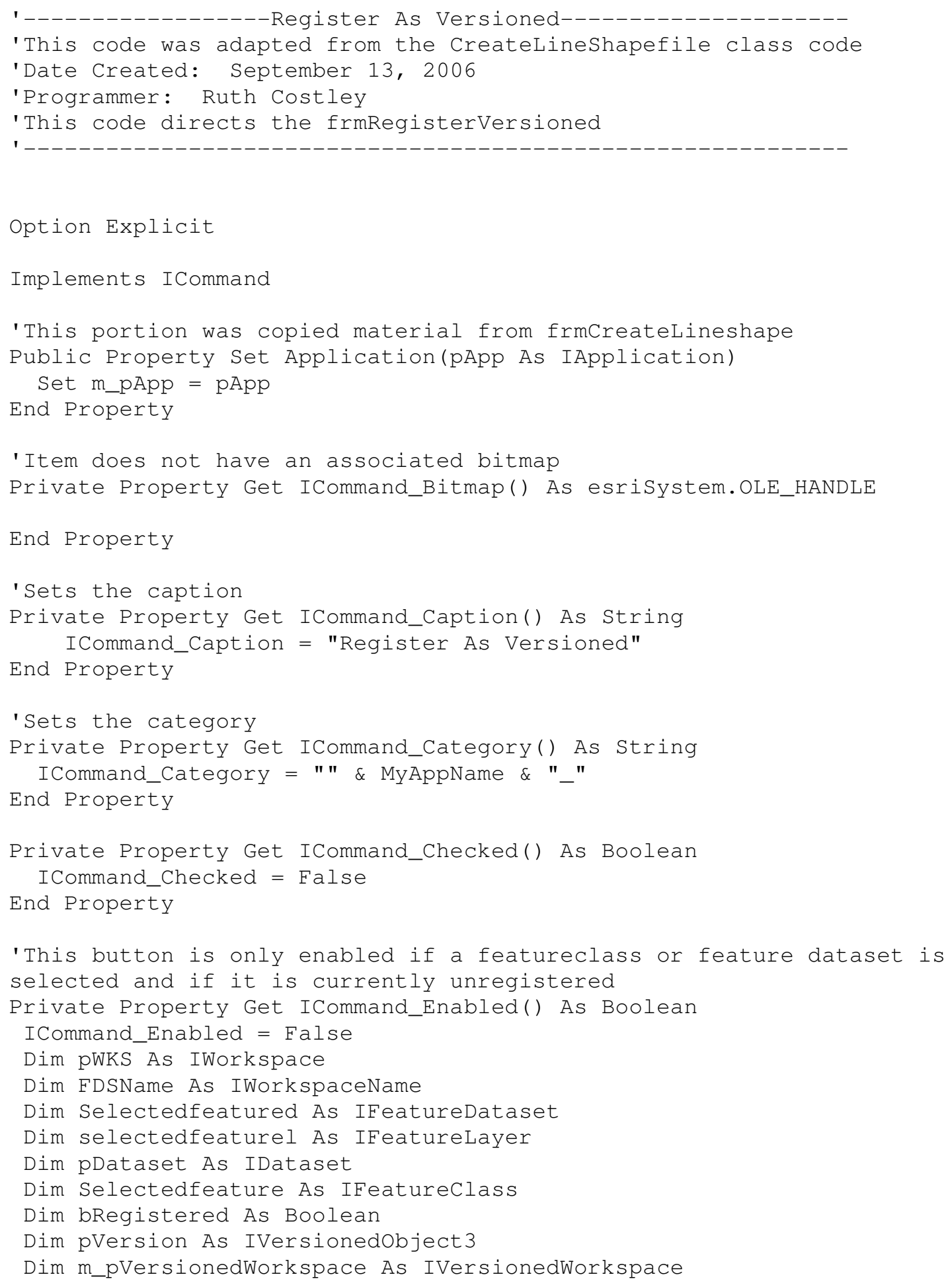




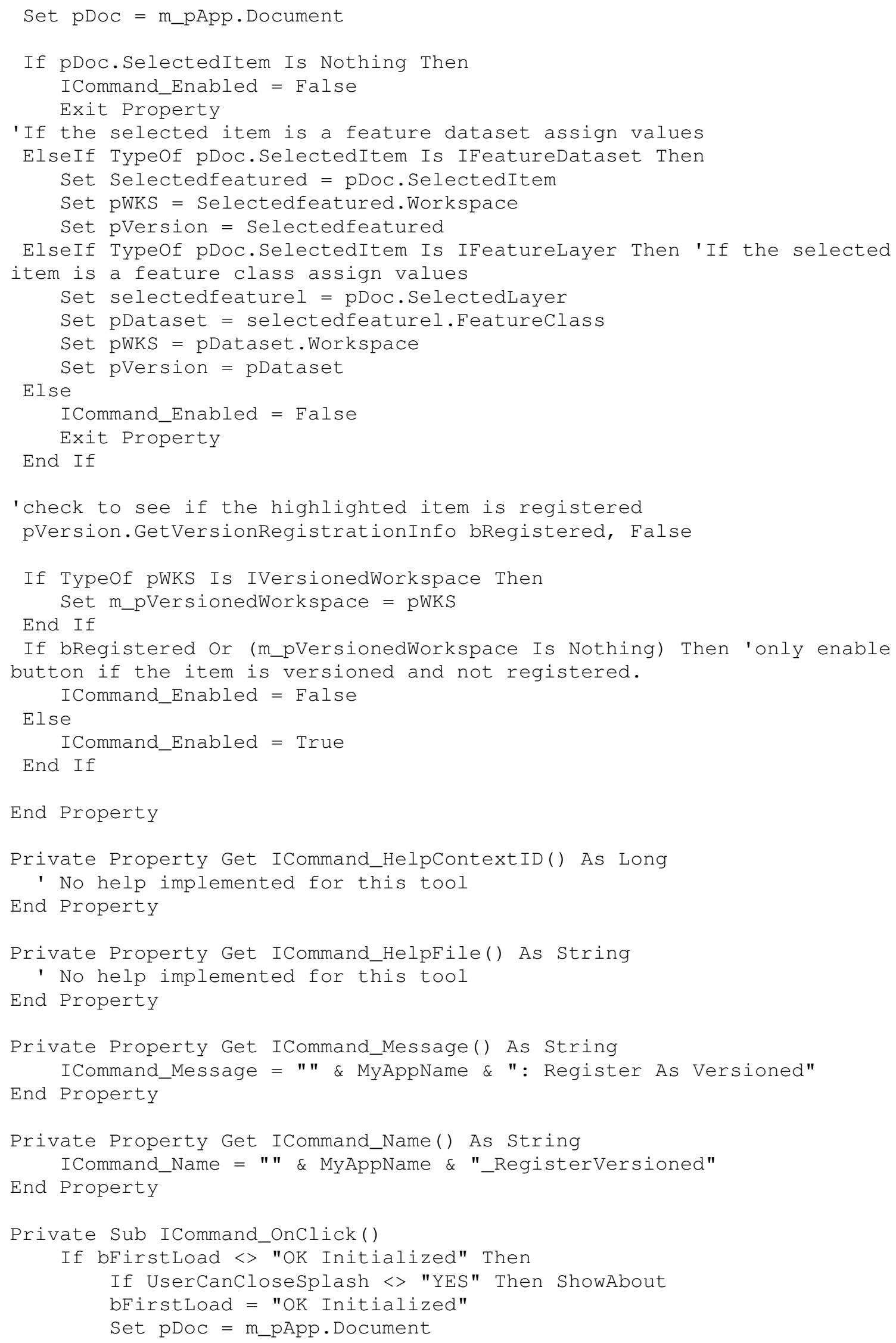




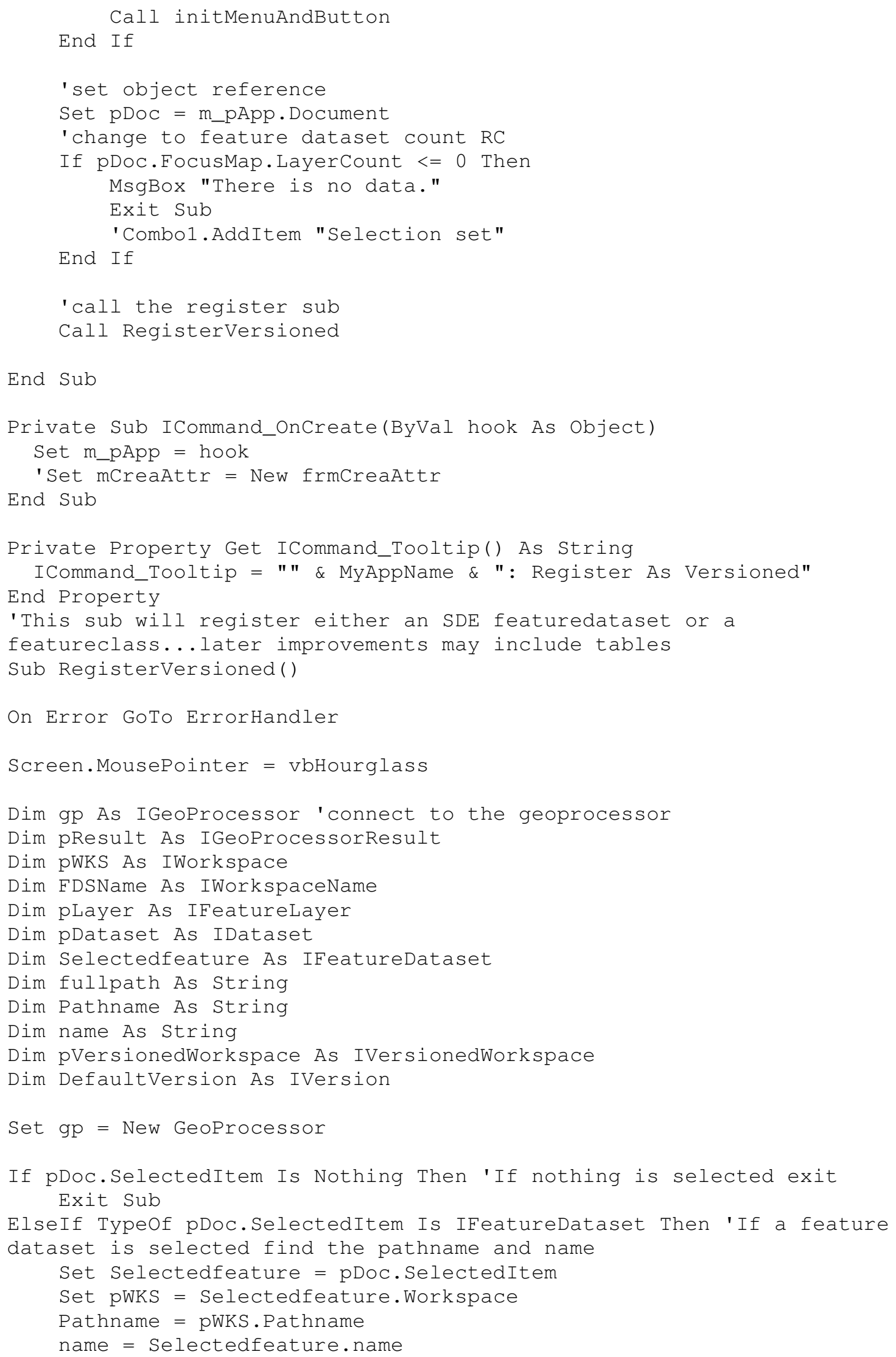




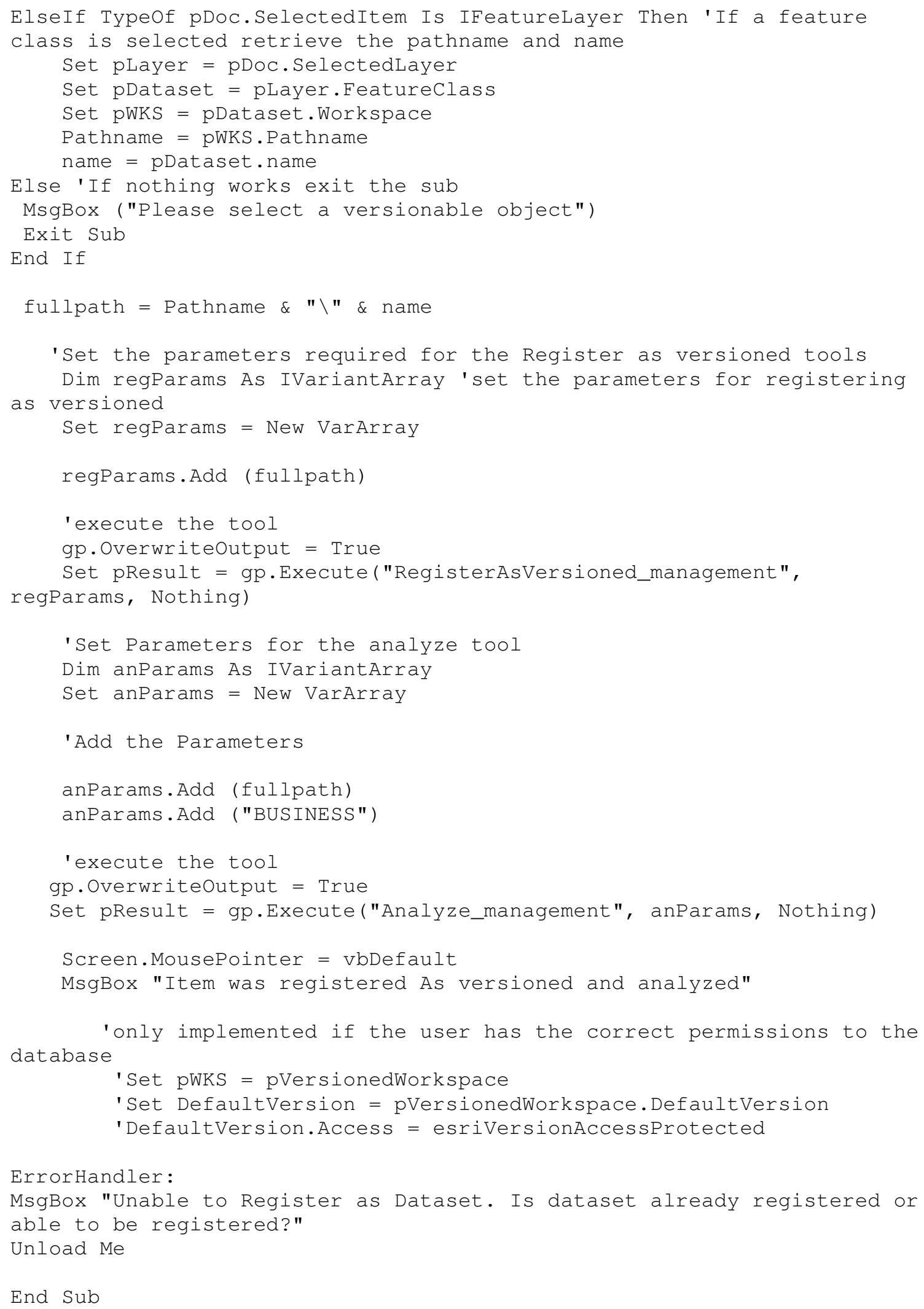




\section{Appendix D - Create Version}

This is the code in createversion.cls. This code will operate the Create Version submenu on the Modeling toolbar.

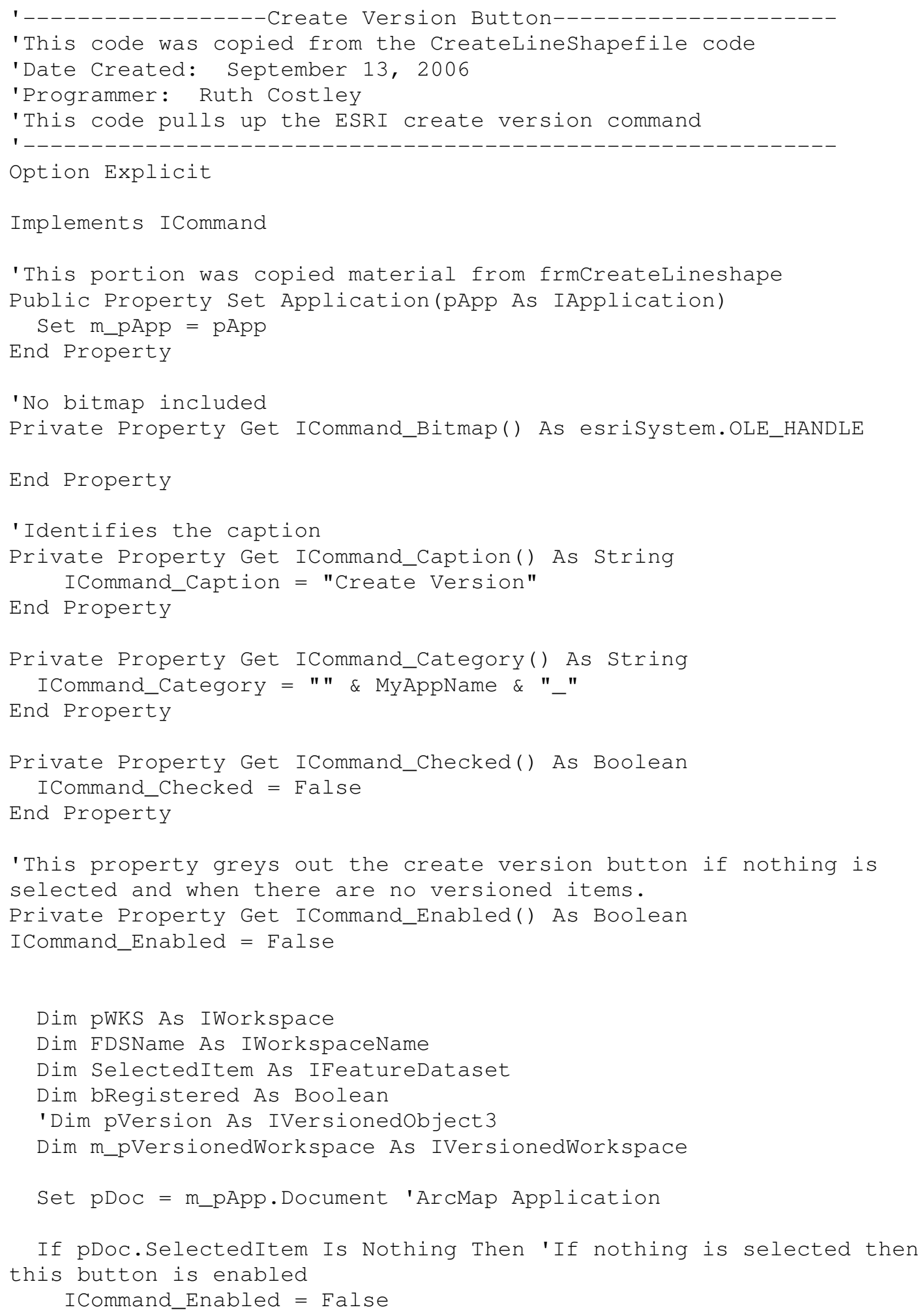




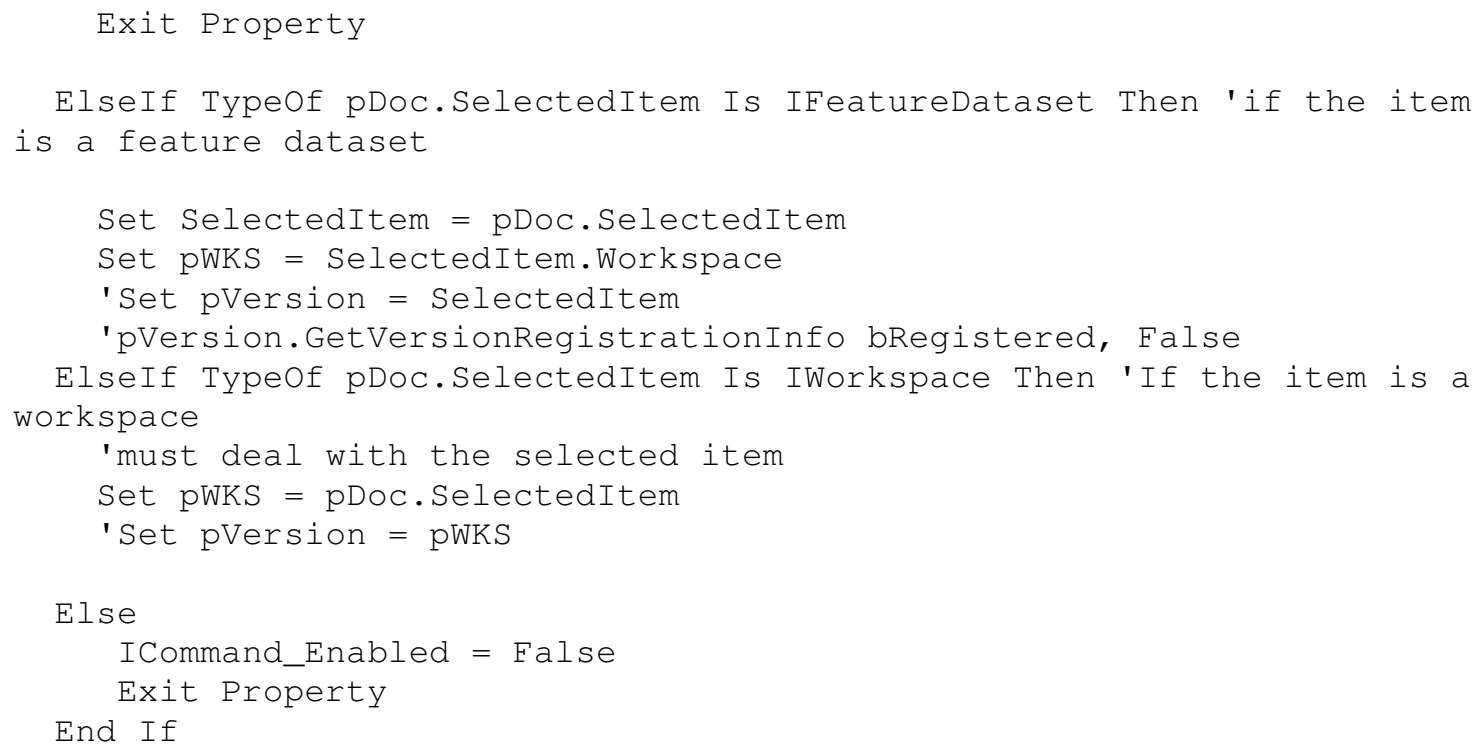




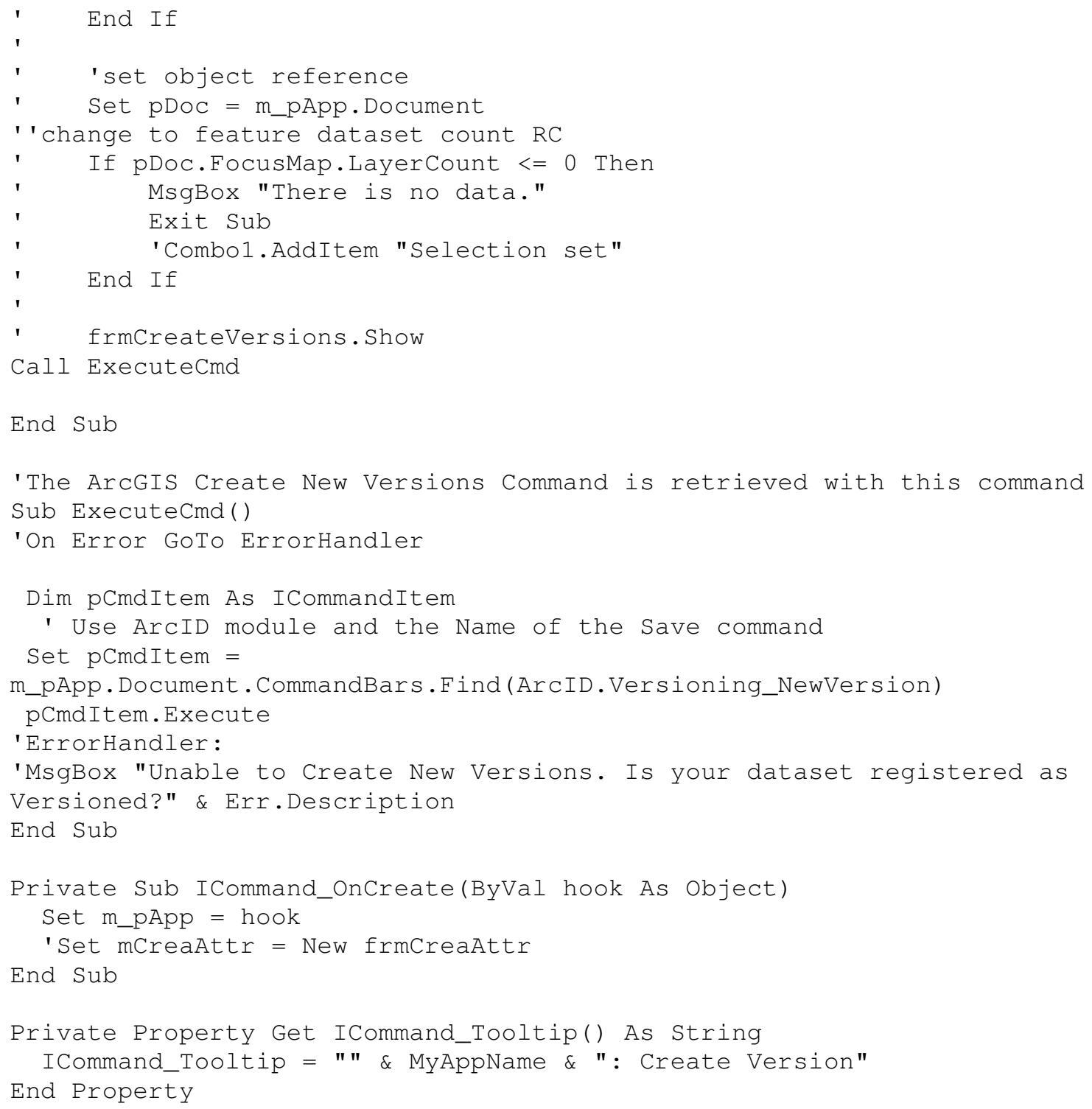





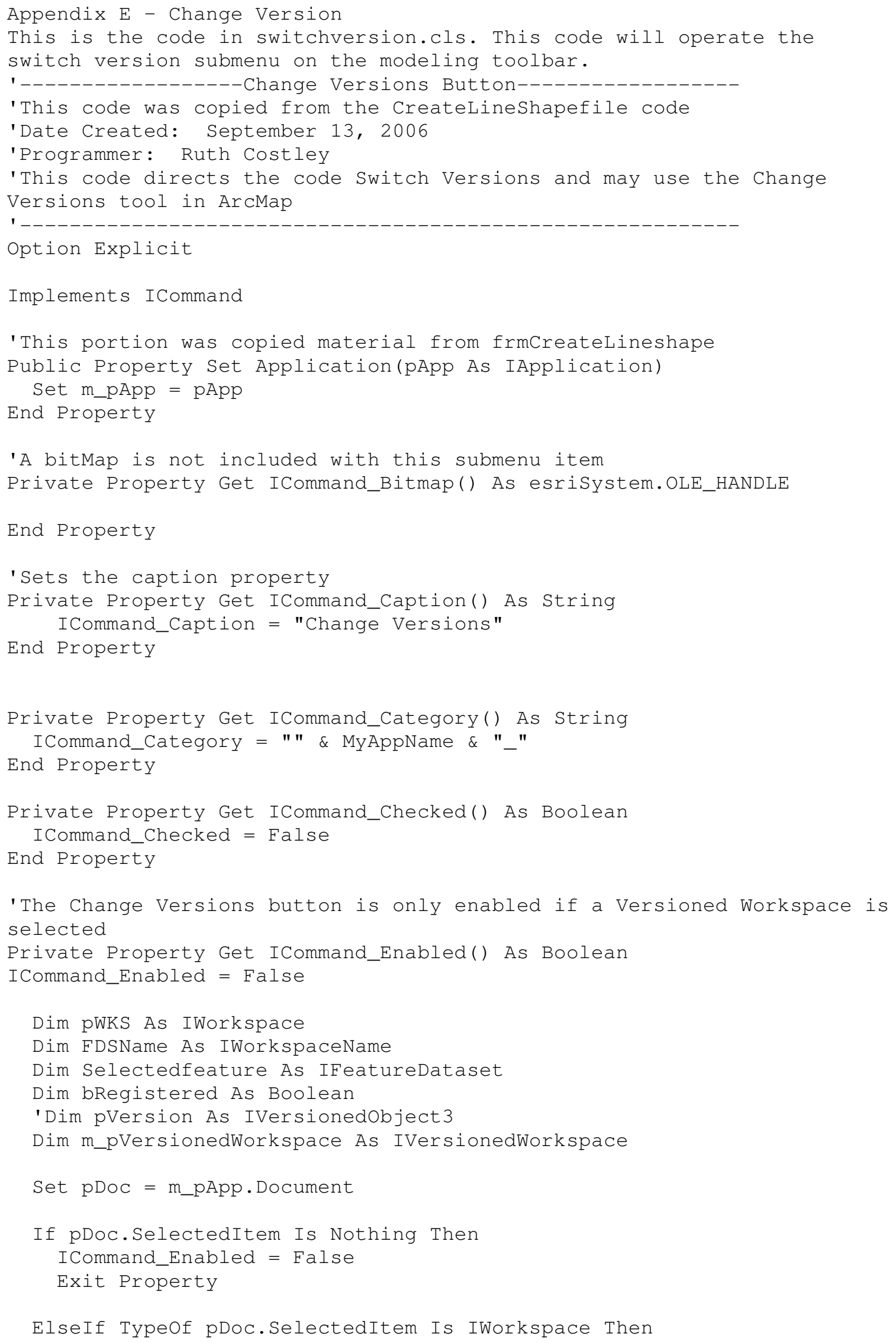




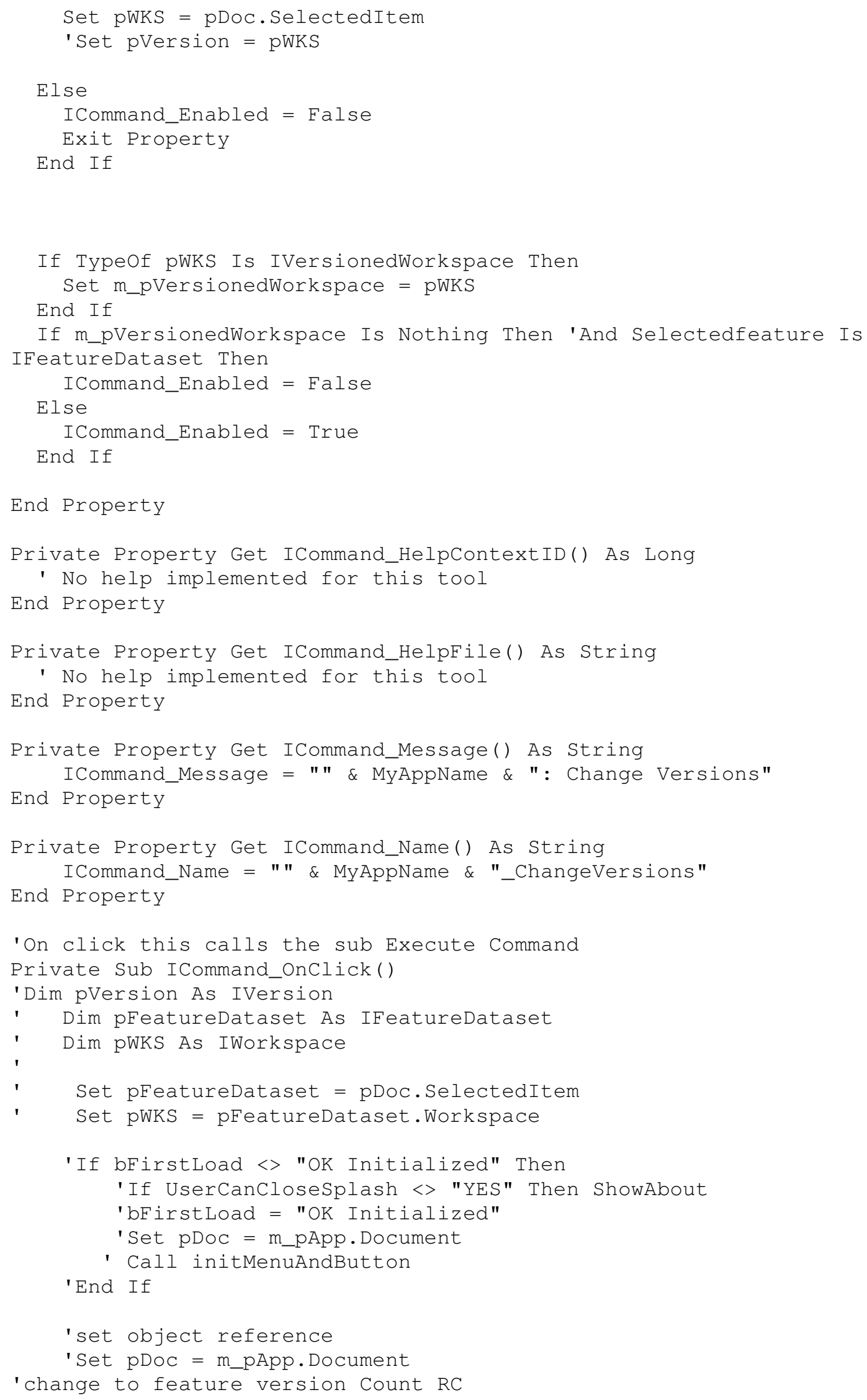




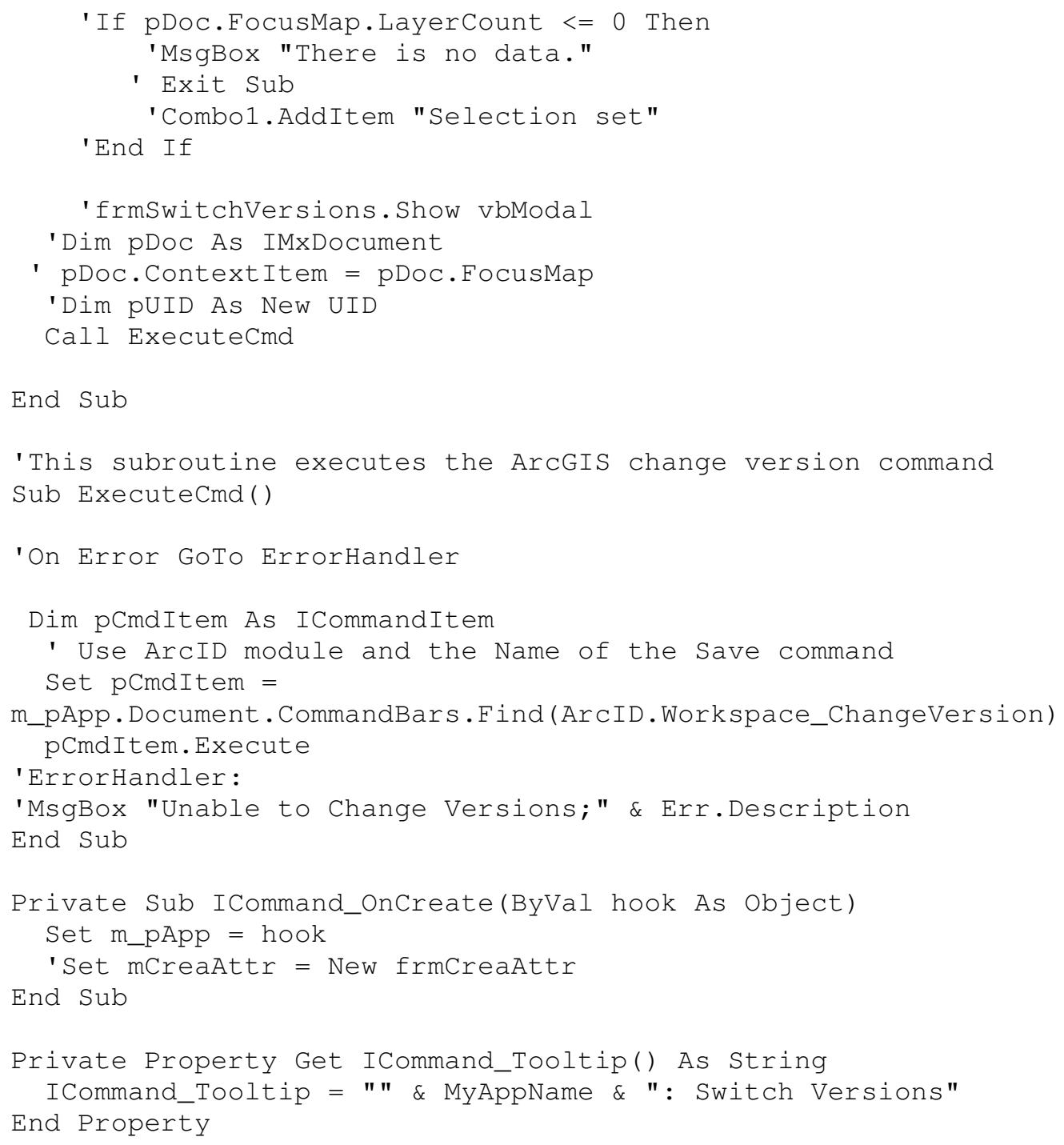





\section{Appendix F - Version Report}

This command is associated with three sets of code: VersionDifferences.cls, frmVersions.frm, and modVersioning.bas. This command was adapted from an ESRI developer's sample.

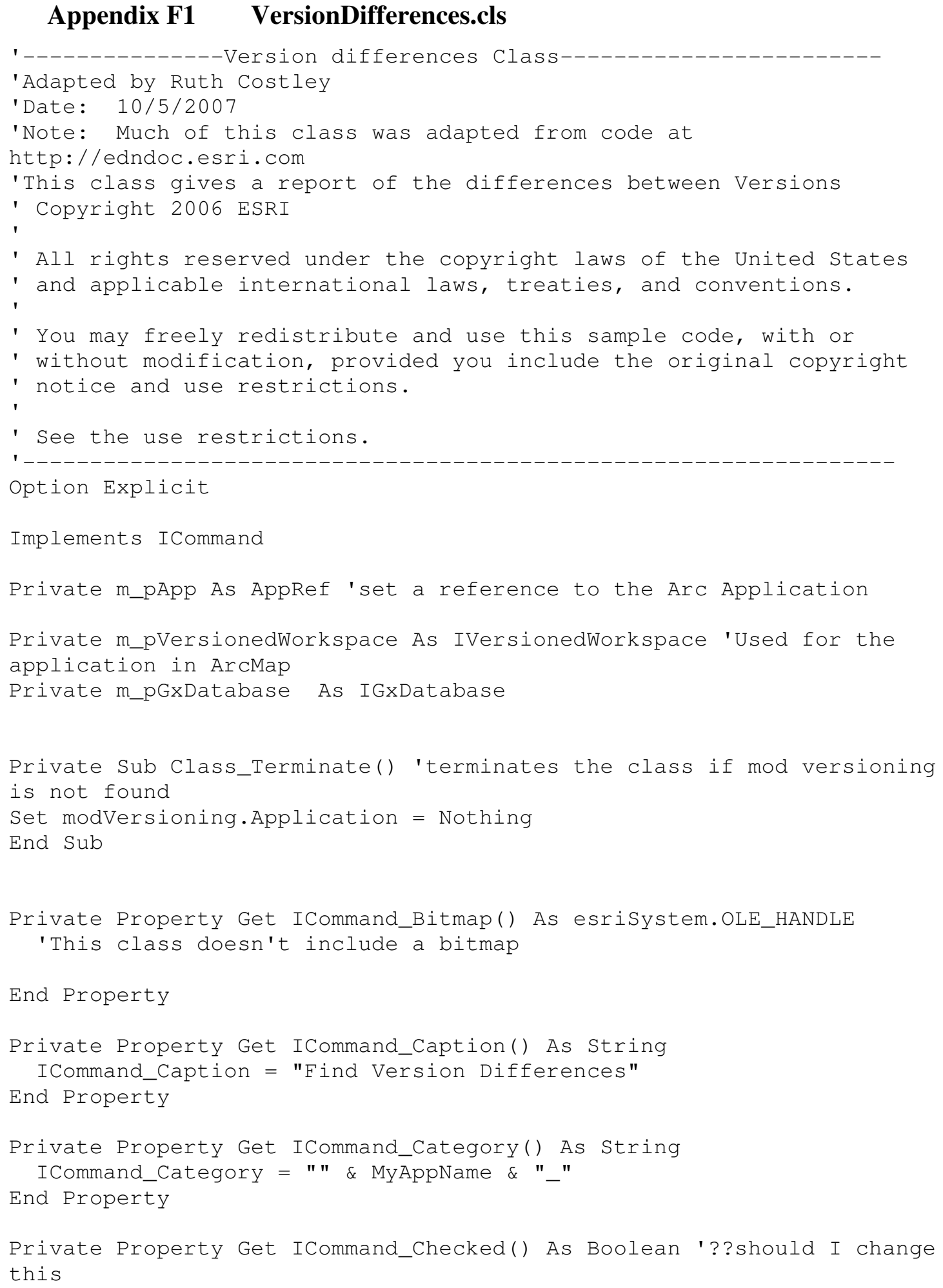




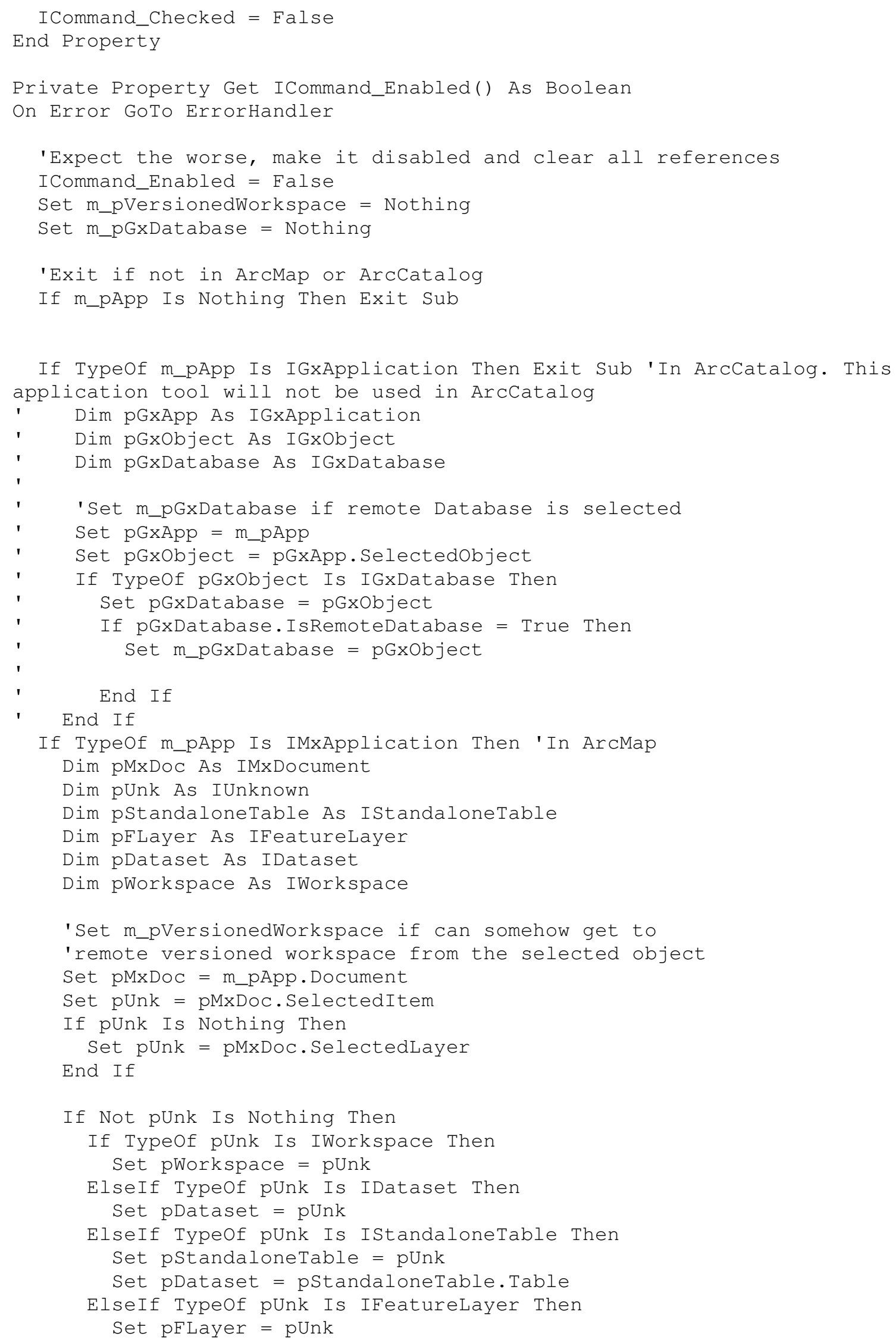




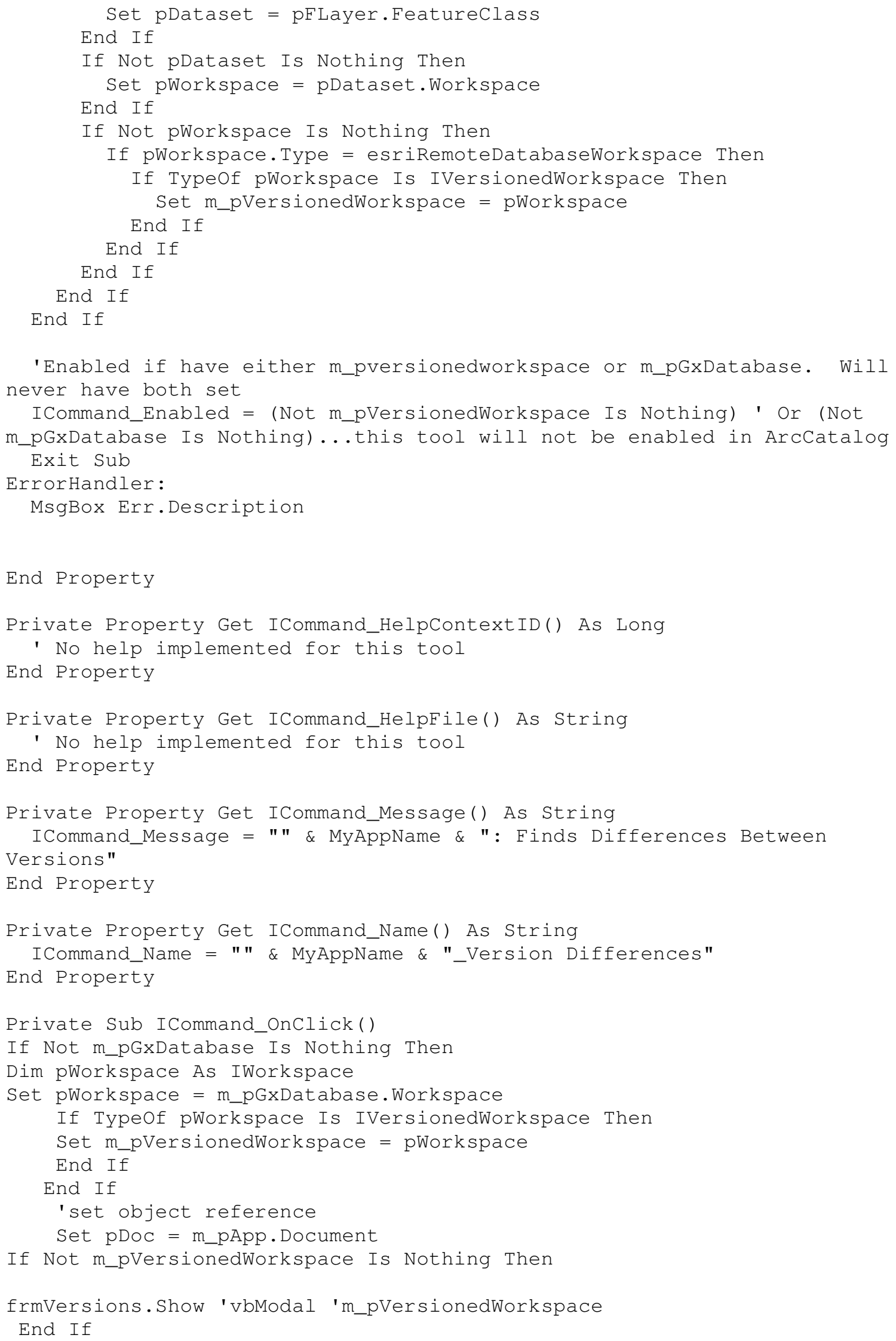




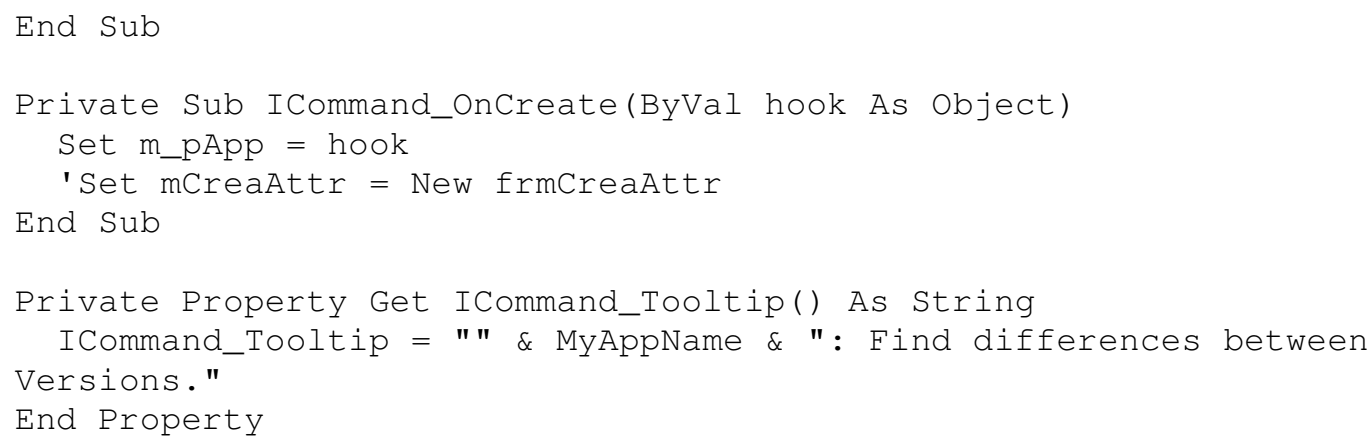

\section{Appendix F2 modVersioning.bas}

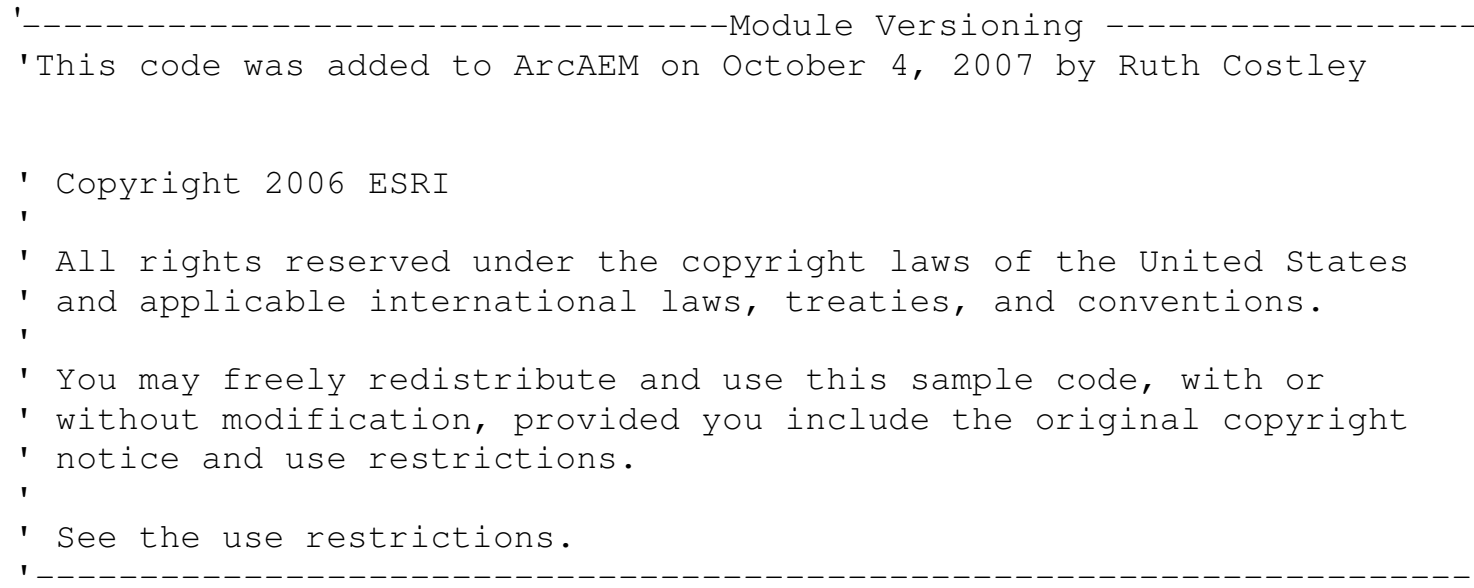




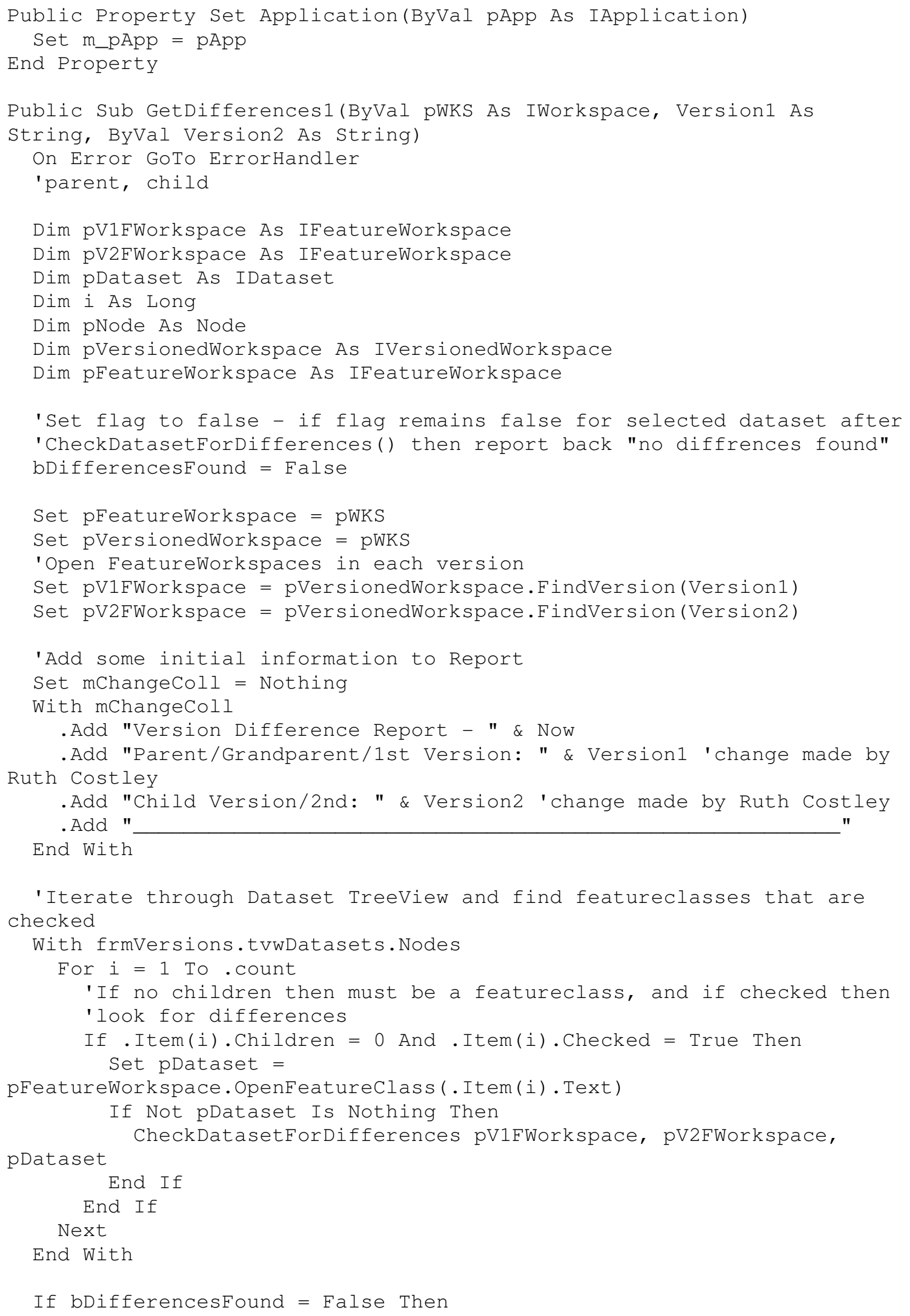




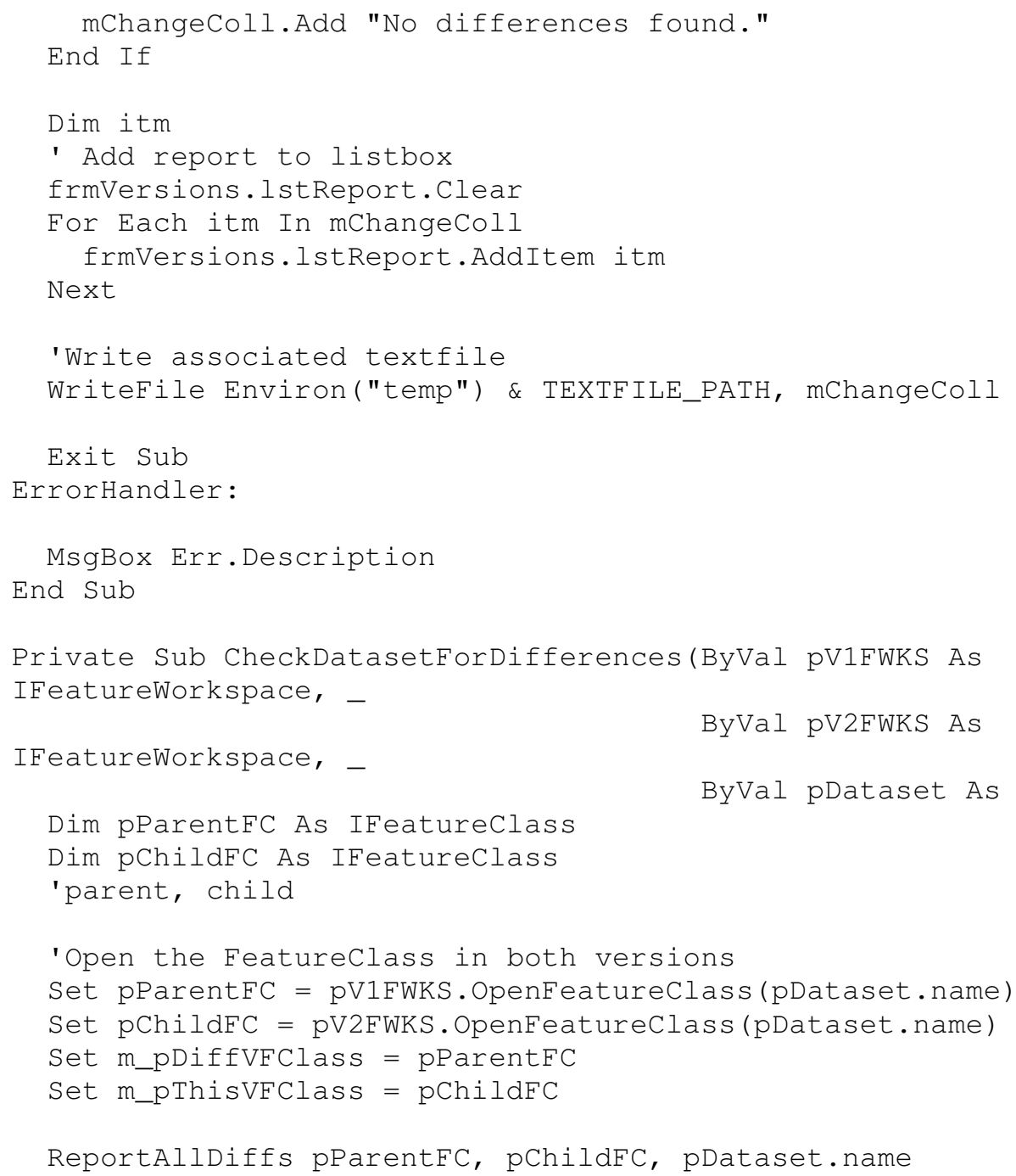




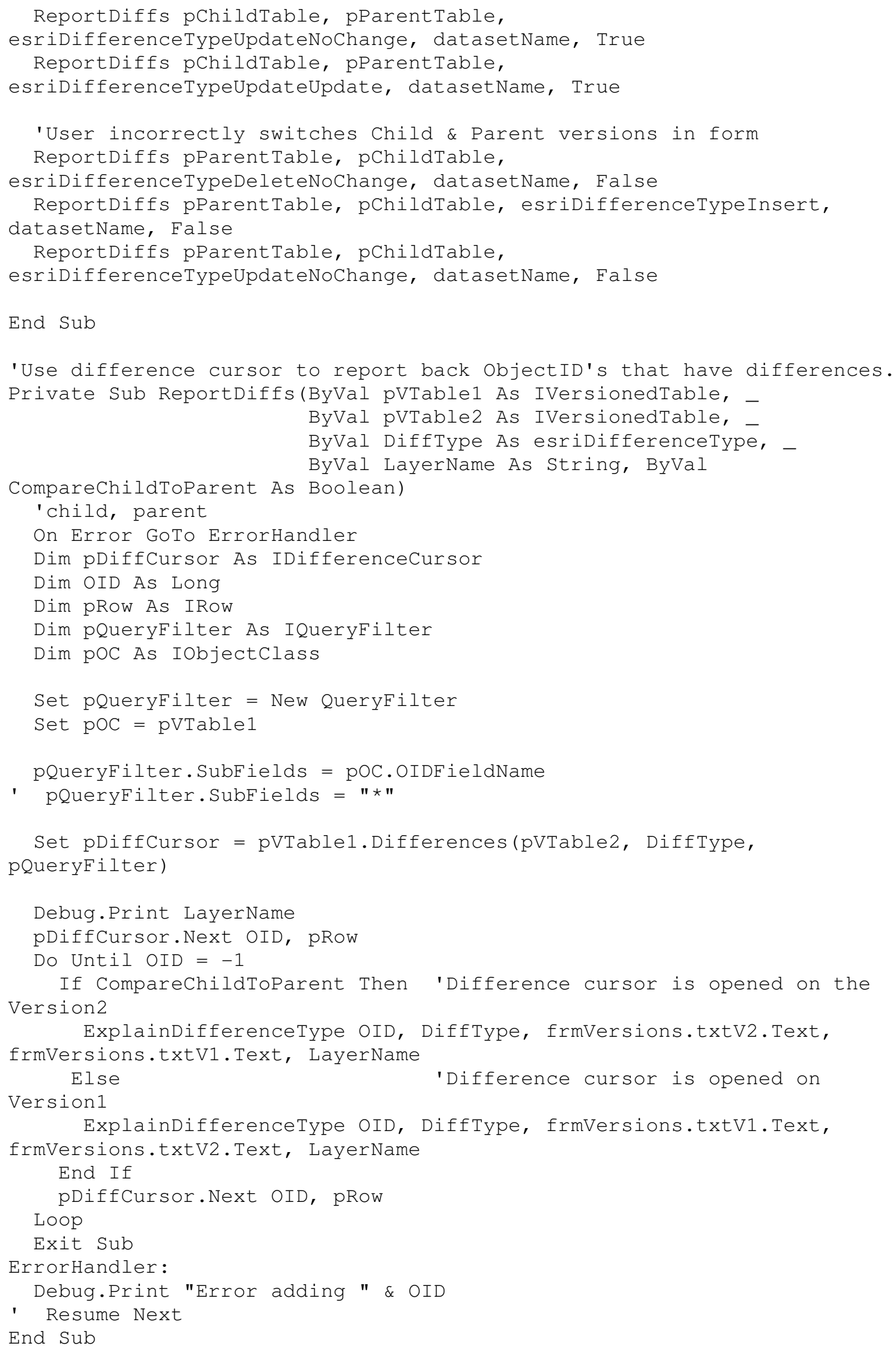




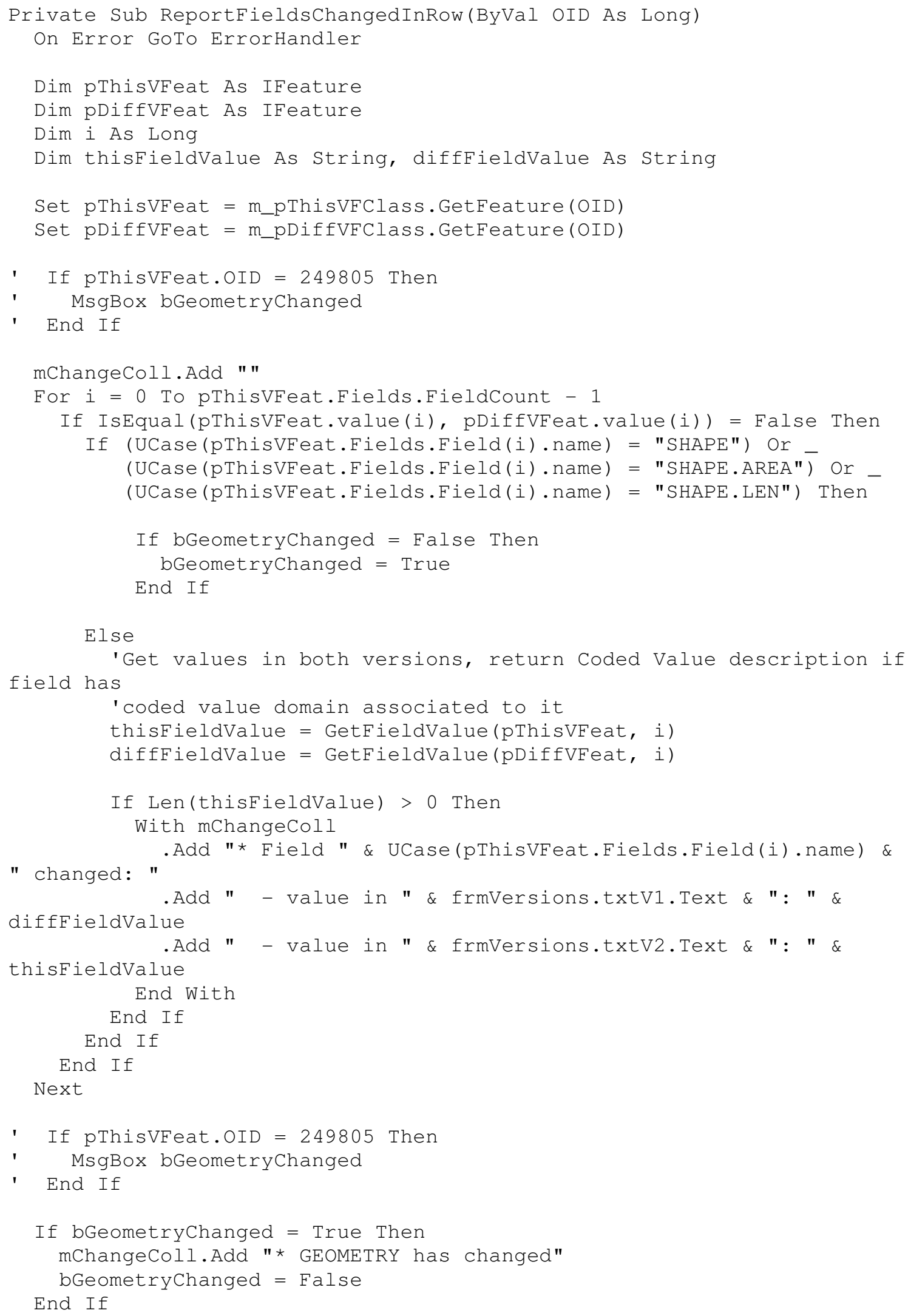




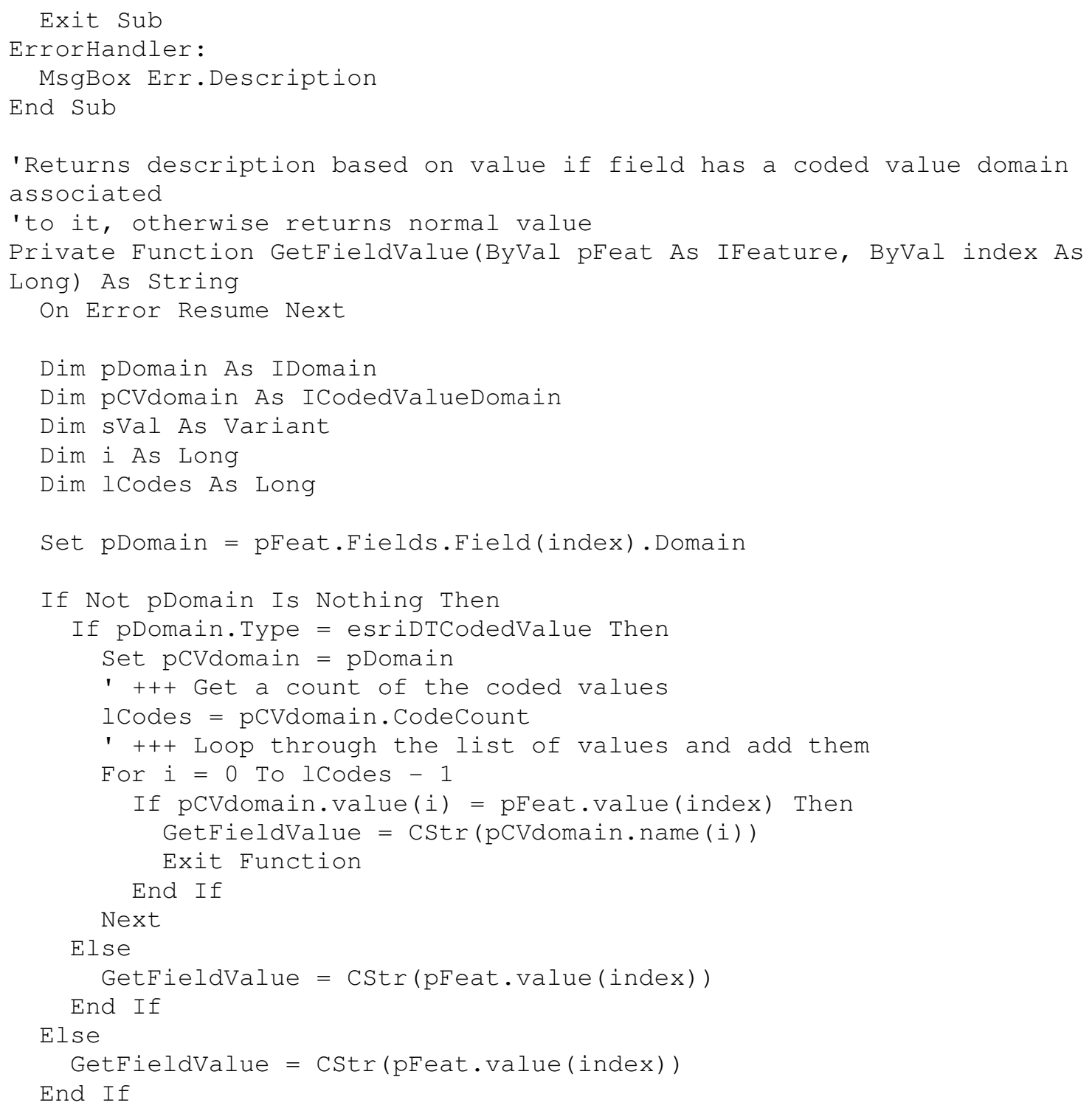




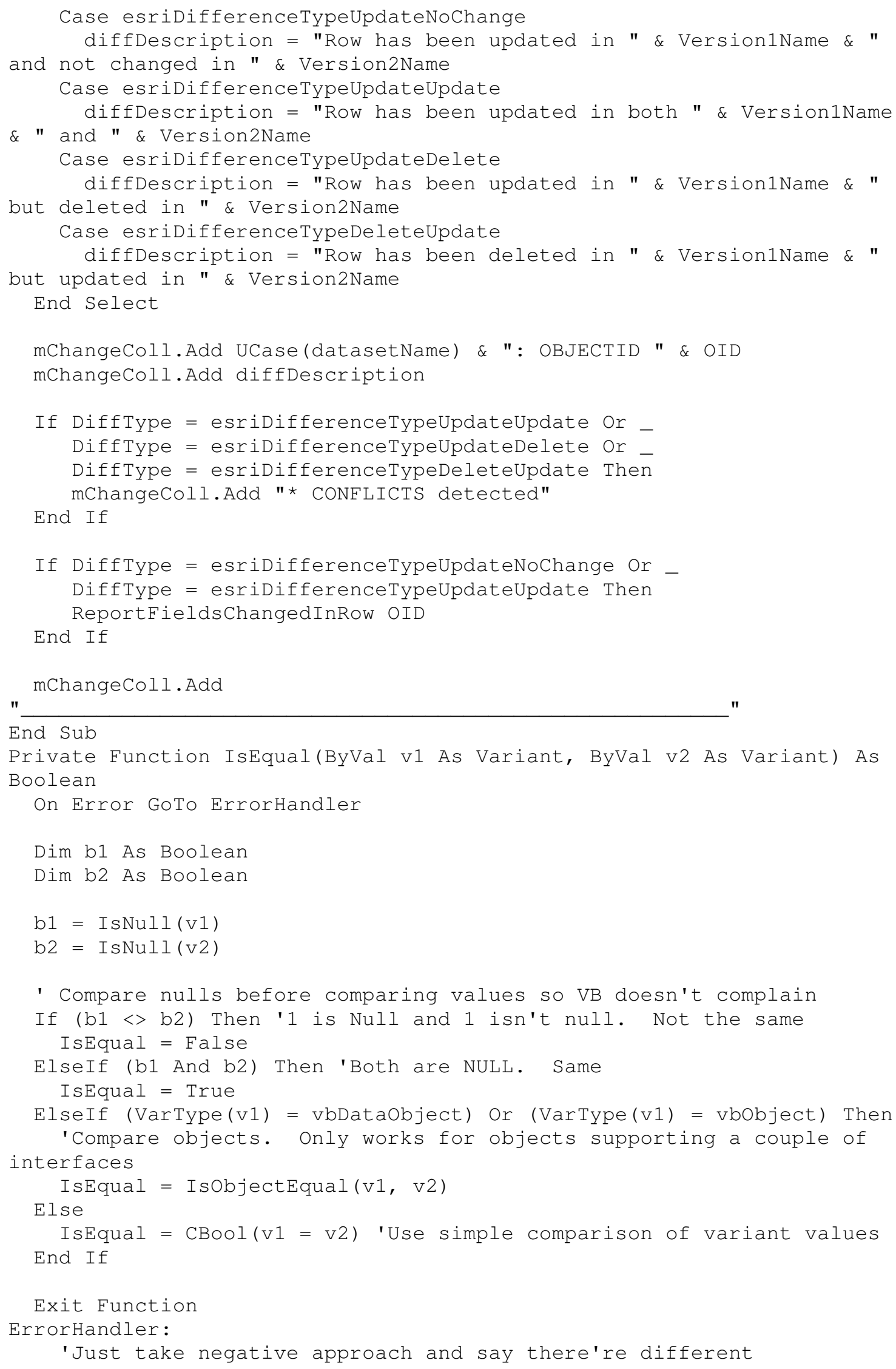




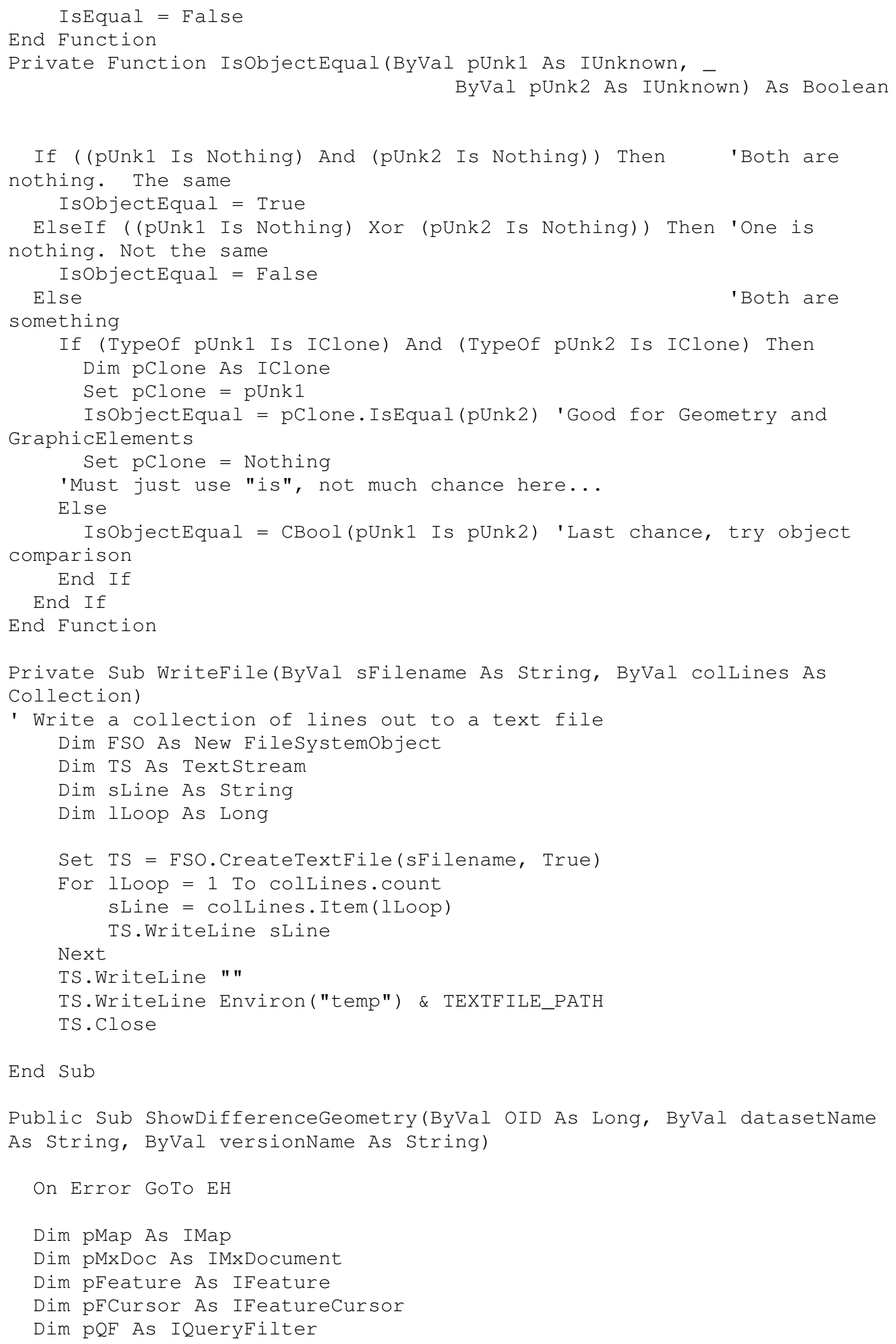




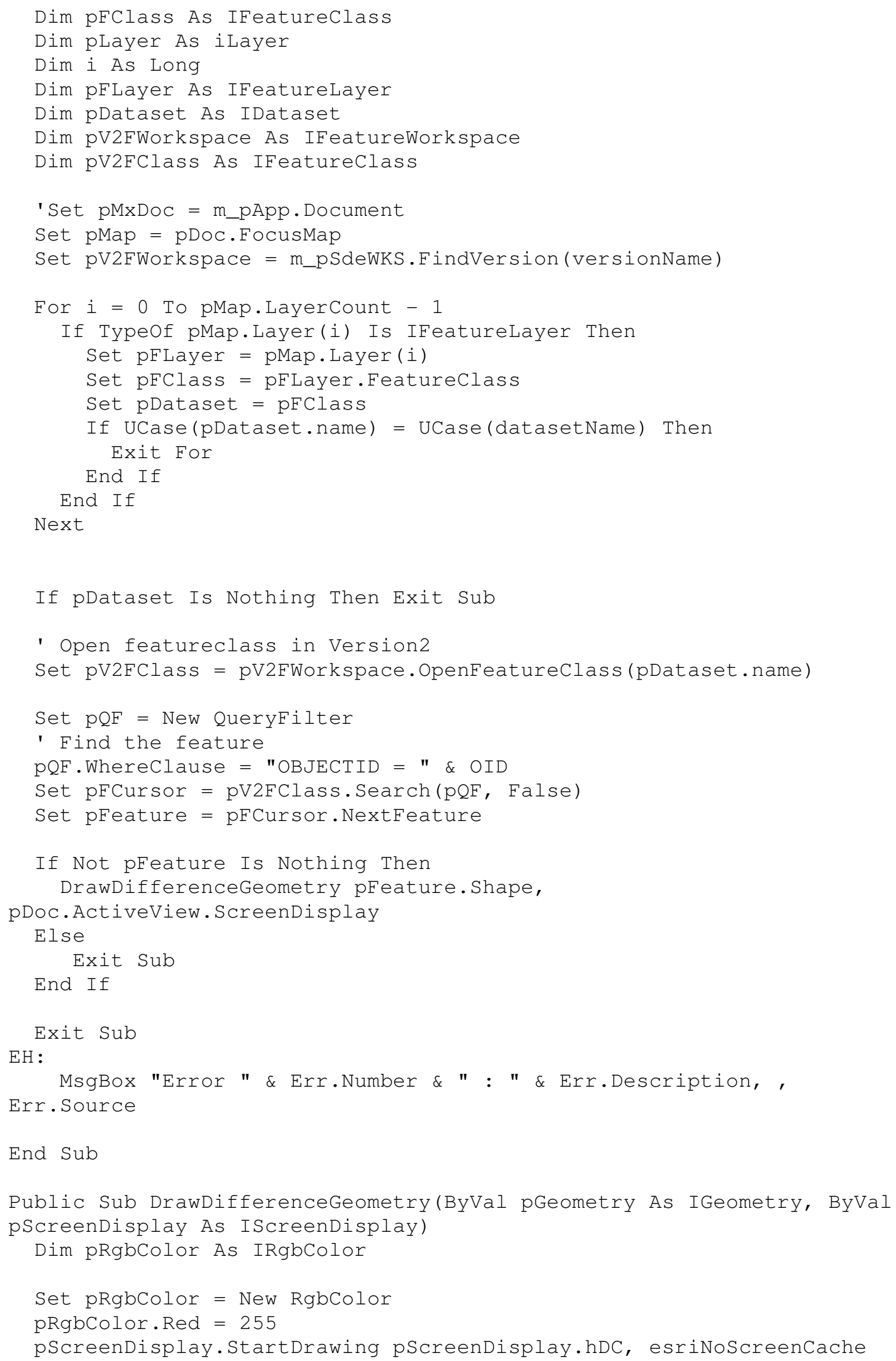




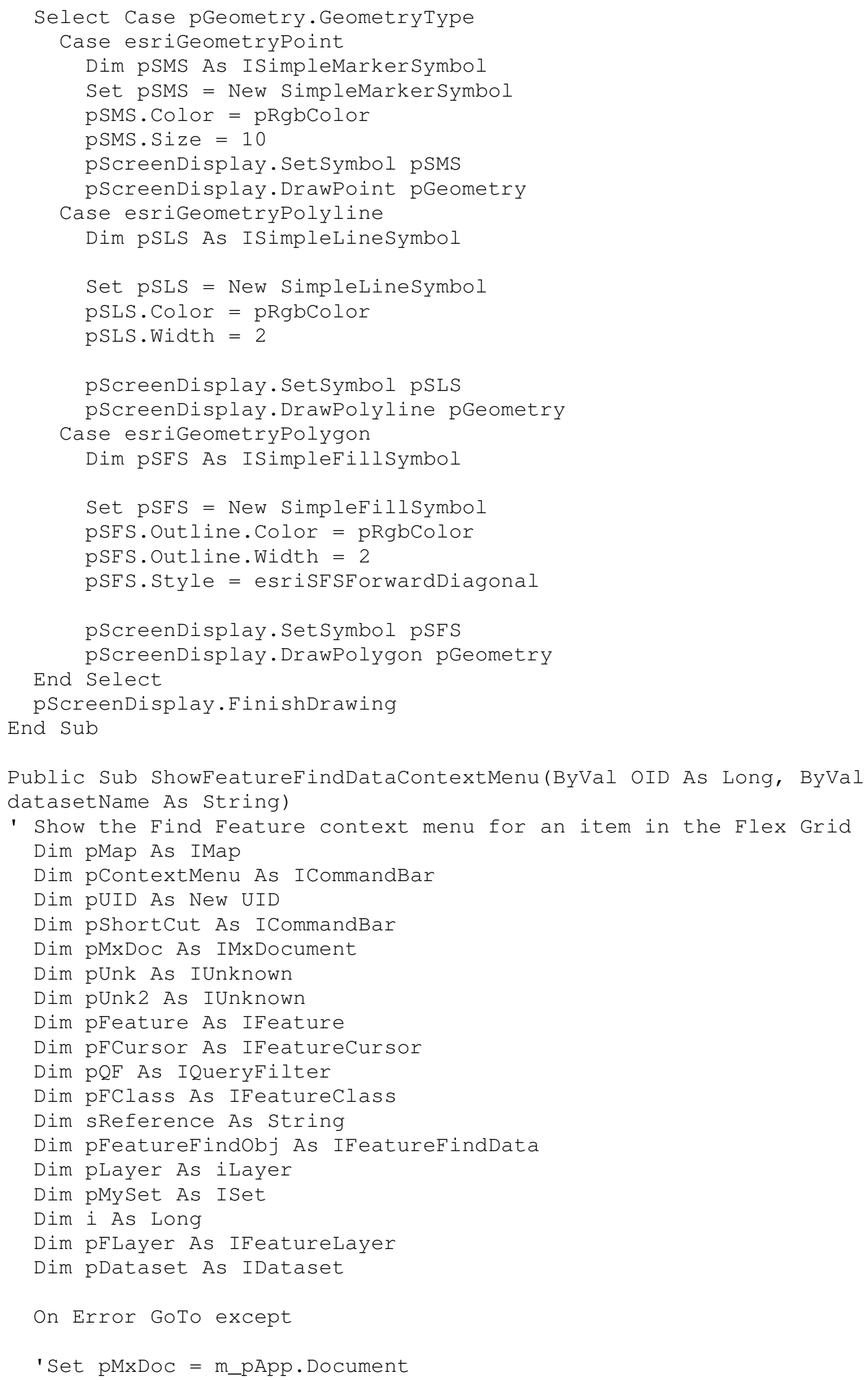




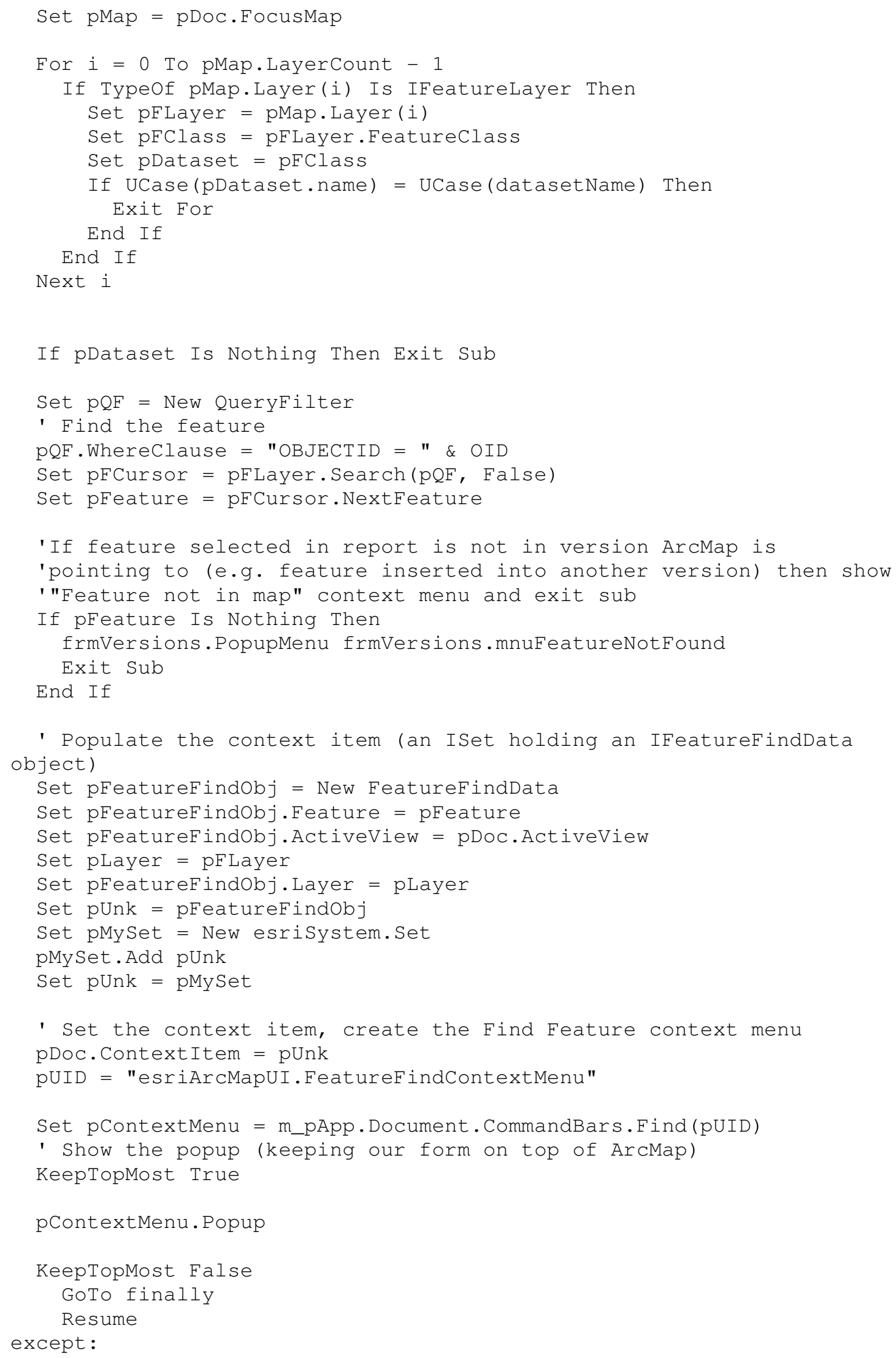




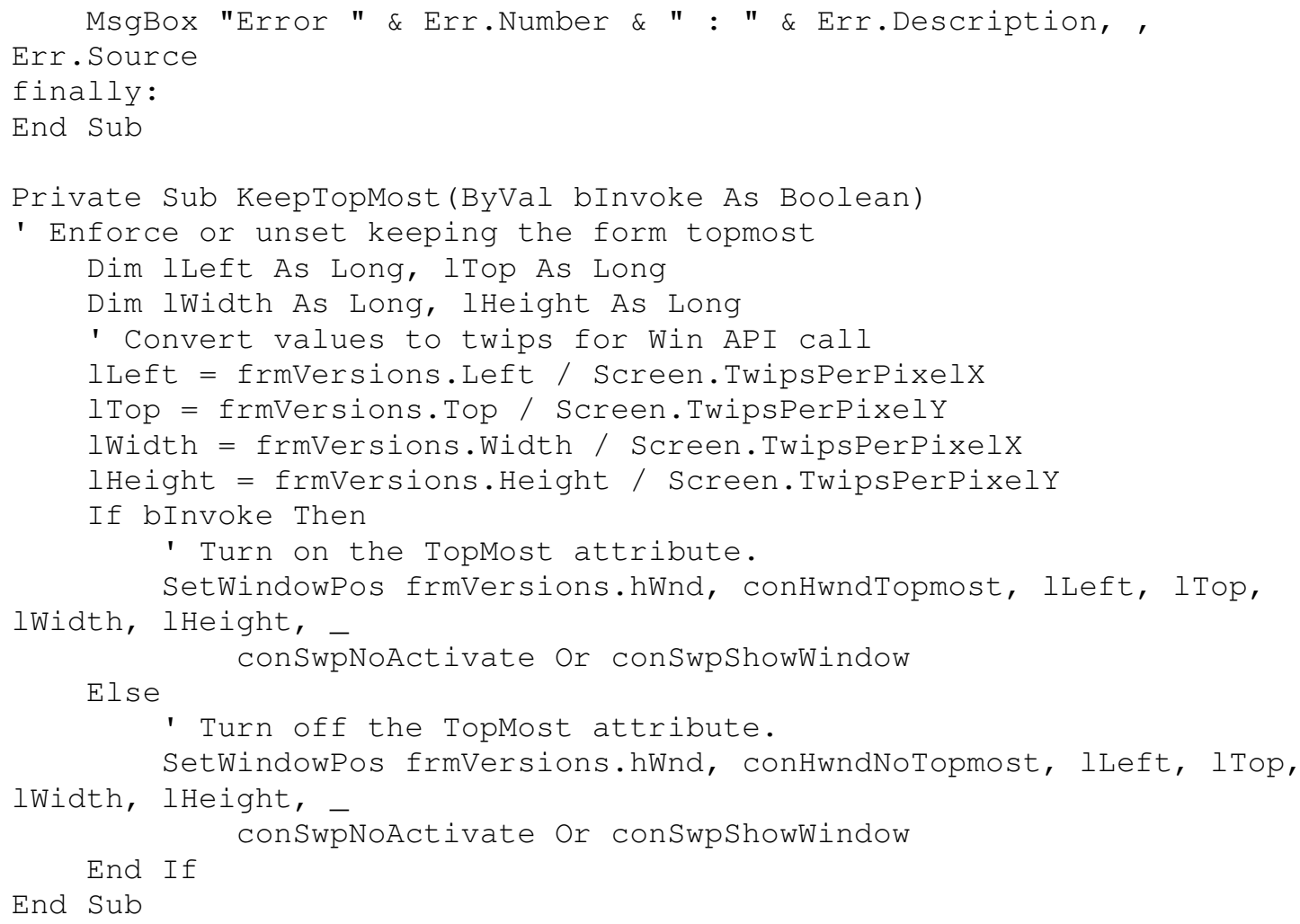

\section{Appendix F3 frmVersions.frm}

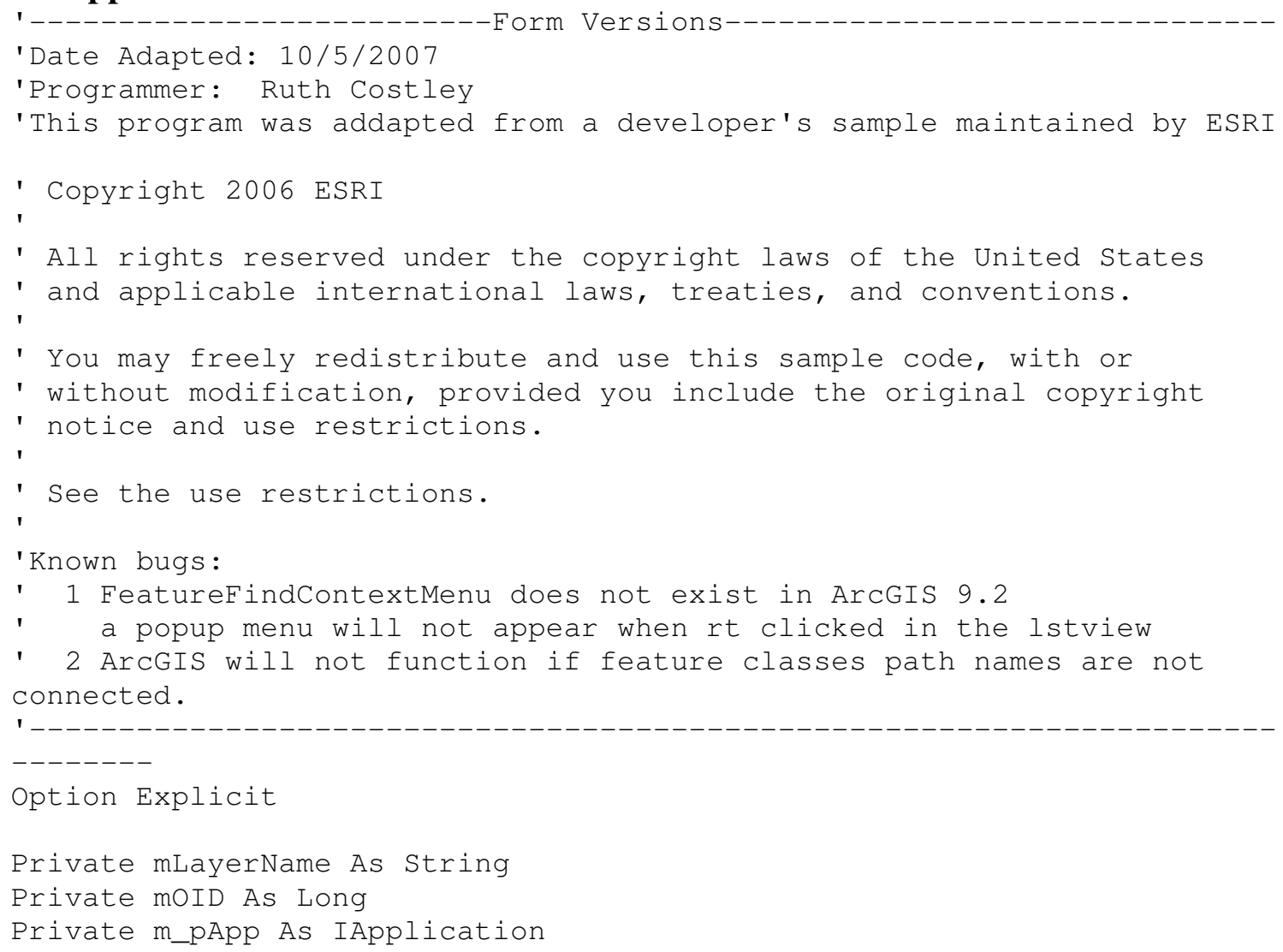




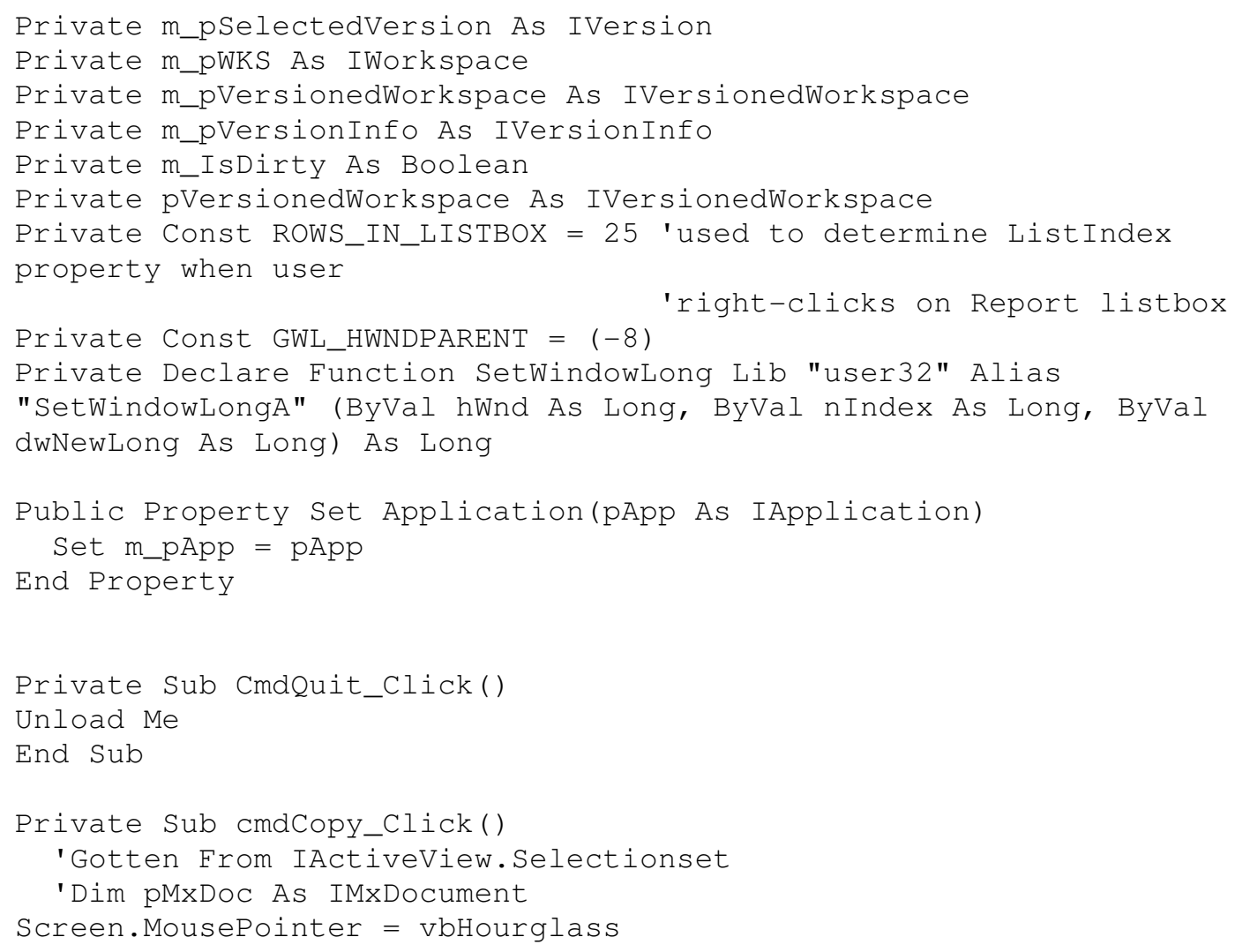




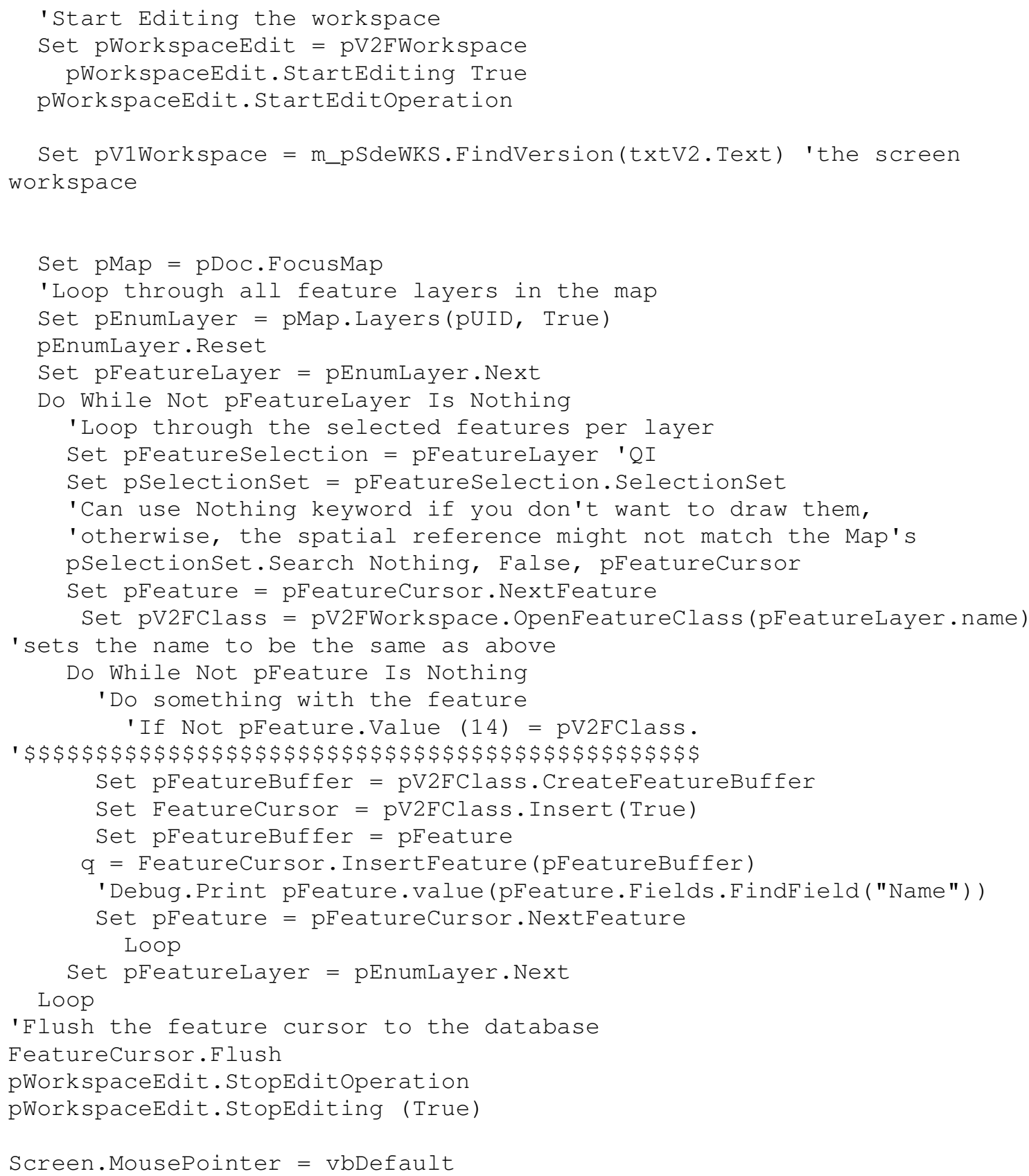




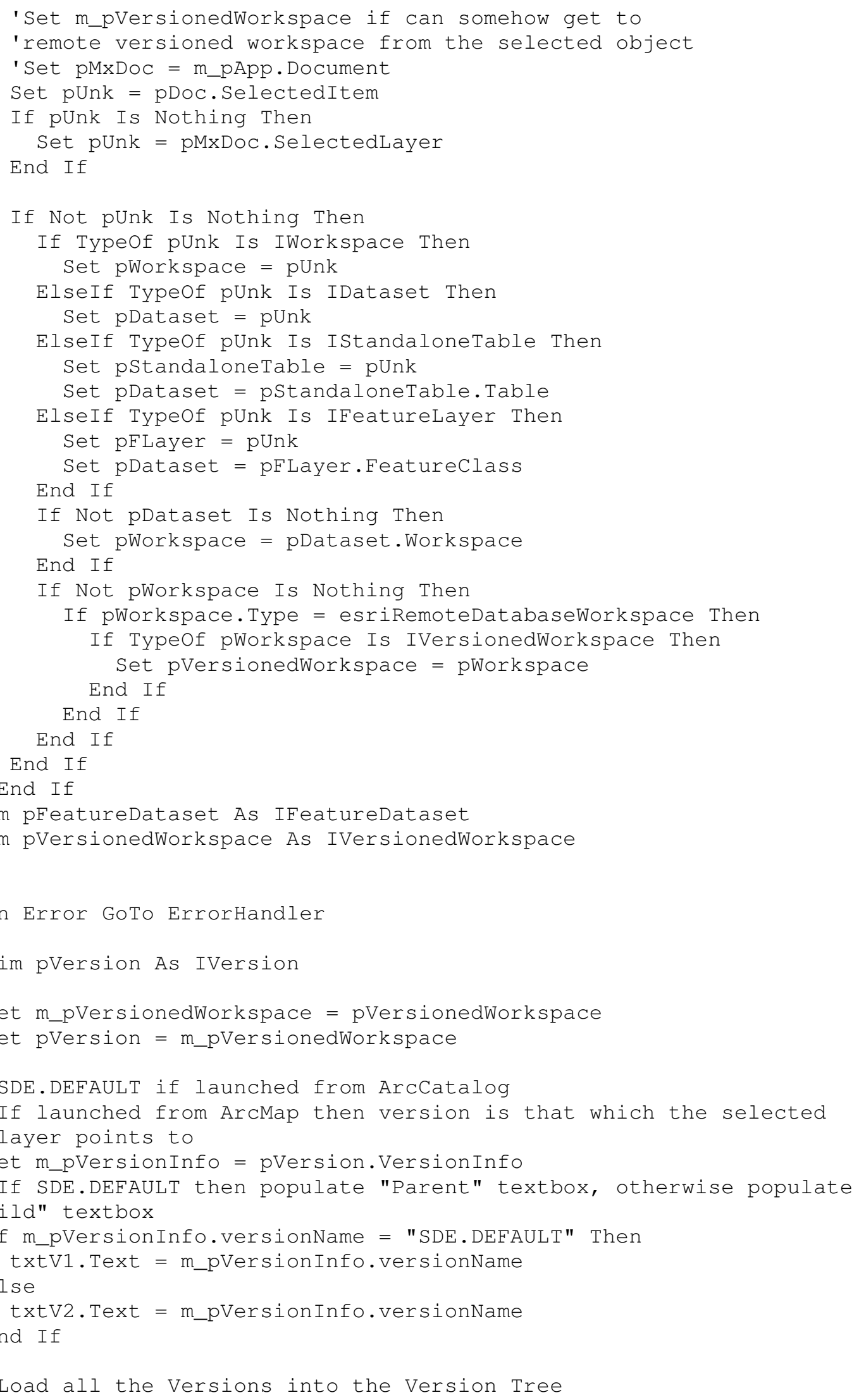




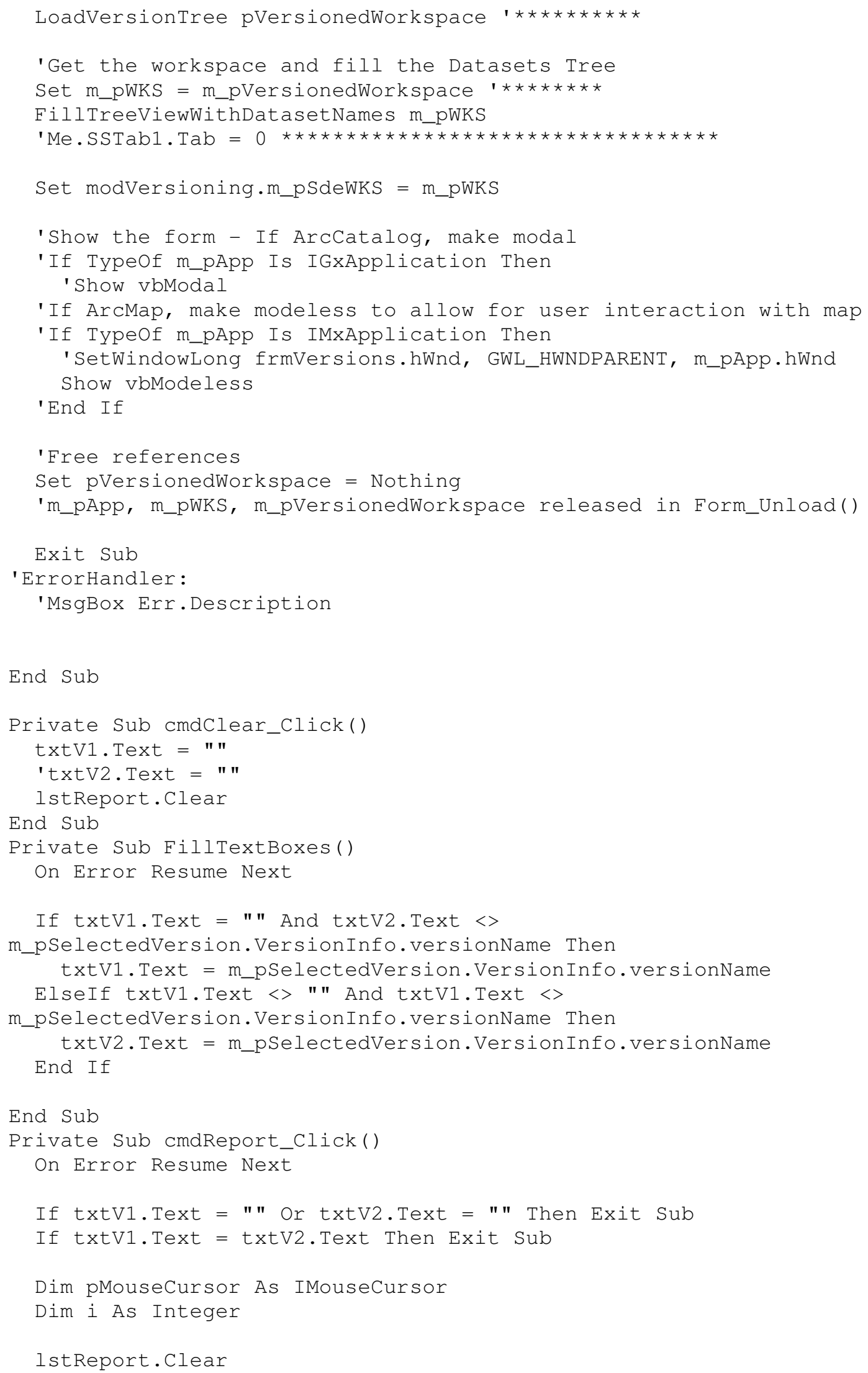




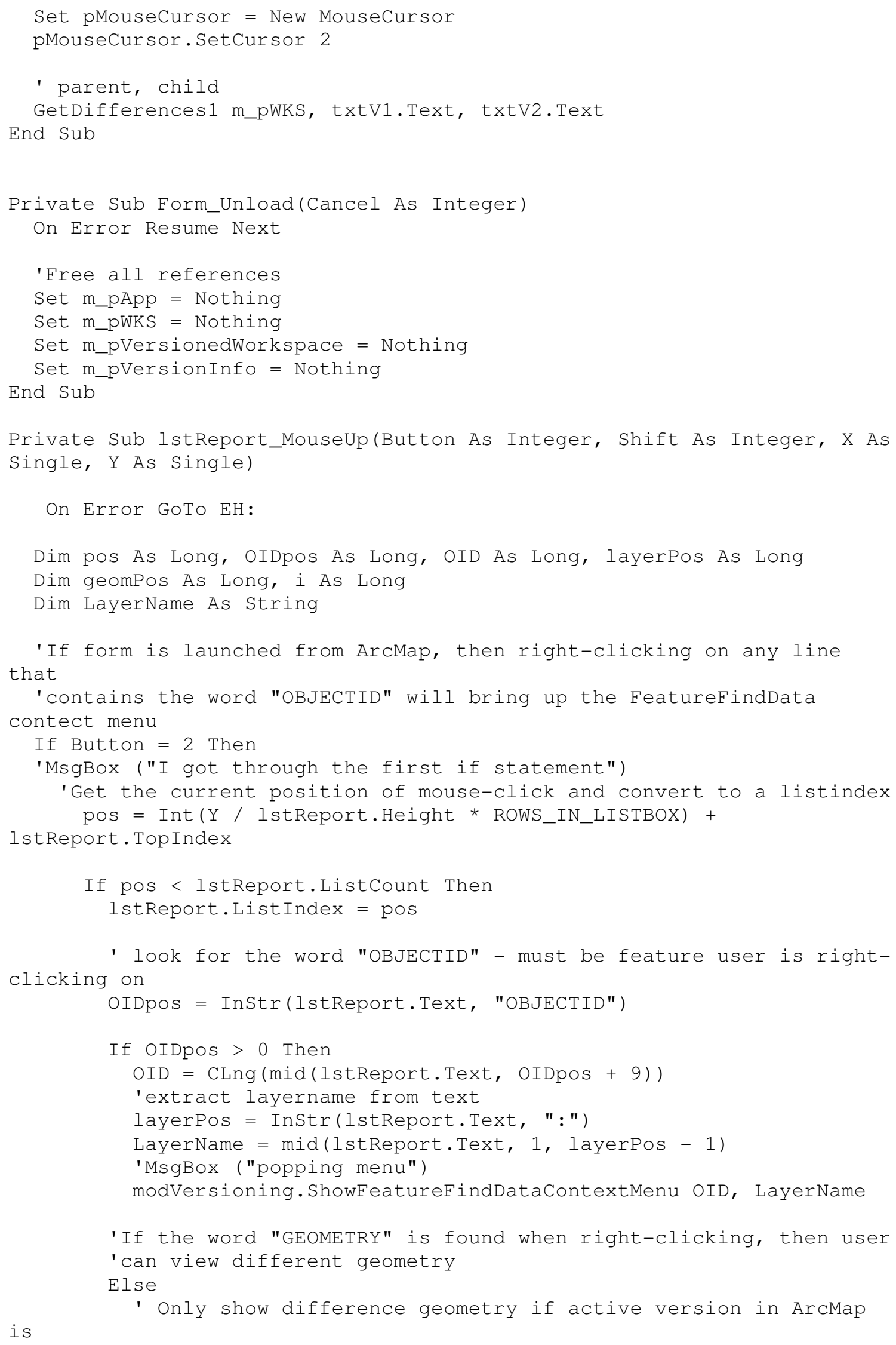




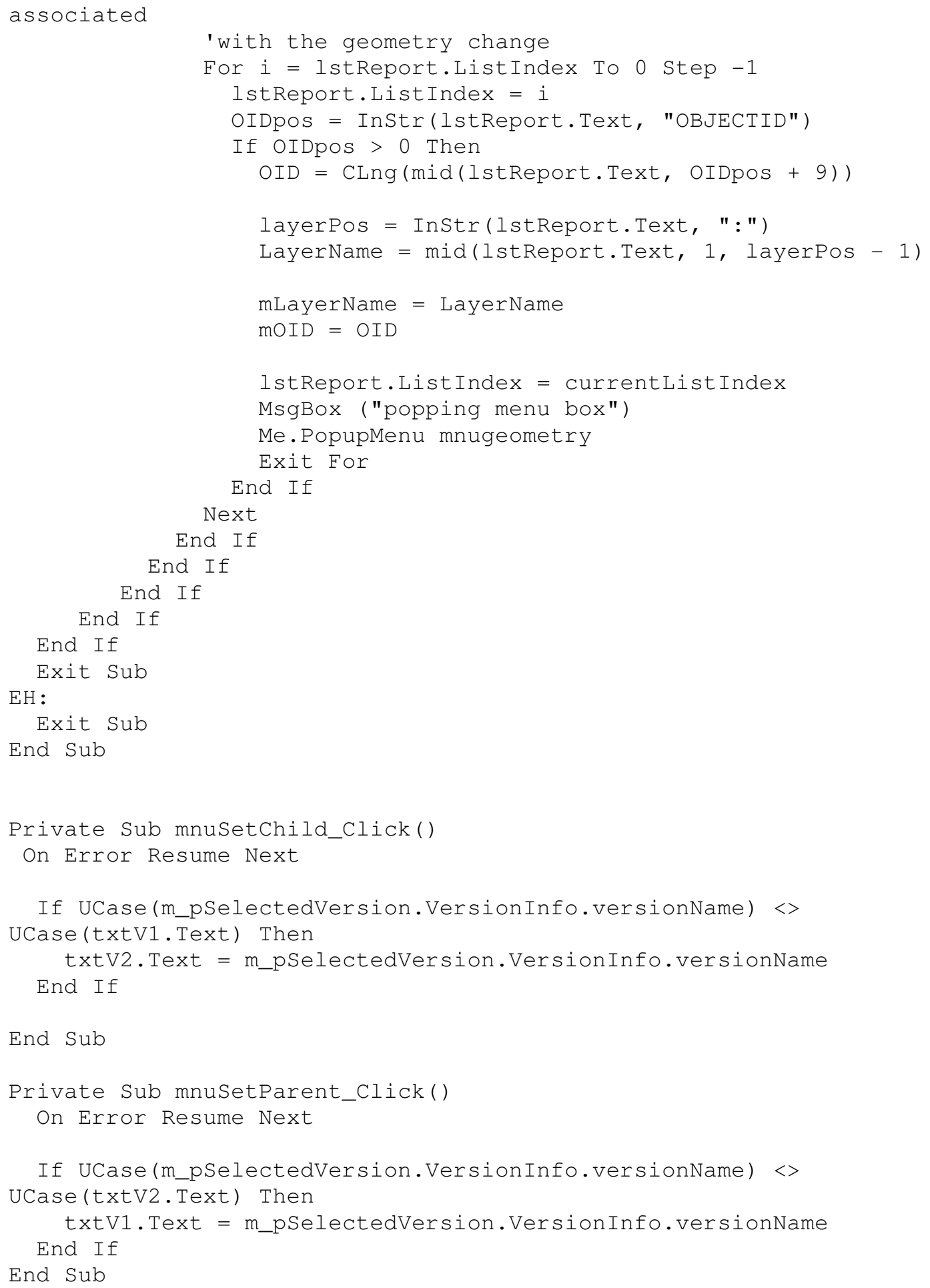




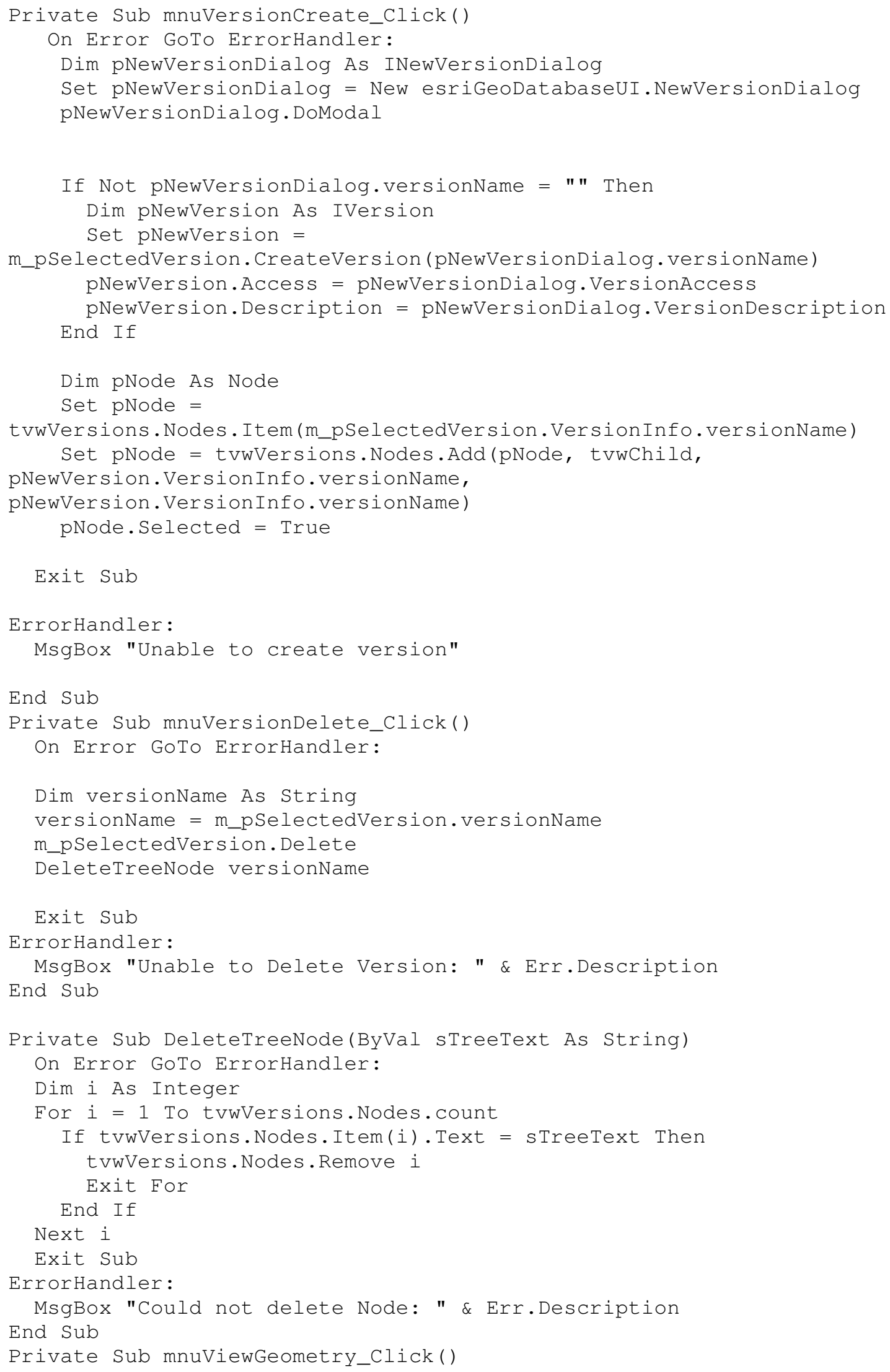


On Error Resume Next

ShowDifferenceGeometry moID, mLayerName, txtV1.Text End Sub

Private Sub cmdCancel_click()

On Error Resume Next

Unload $\mathrm{Me}$

End Sub

Private Sub cmoTextFile_click()

On Error Resume Next

Dim retval As Double

retval = Shell (NOTEPAD_EXE \& " " \& Environ("temp") \& TEXTFILE_PATH, vbNormalFocus)

'retval = Shell (NOTEPAD_EXE \&, vbNormalFocus)

End Sub

'Code from ESRI

'Recursively adds version and all children versions to tree and

'to dictionary holding all the VersionInfo objects

Private Sub AddVersionToTree (ByVal pVersionInfo As IVersionInfo, ByVal pParentNode As Node)

'Recursive function that first adds the parent VersionInfo to

tvwVersions and

'then calls itself recursively for each child VersionInfo

On Error Resume Next

Dim pEnumVersionInfo As IEnumVersionInfo

Dim pNode As Node

'Add the parent VersionInfo to the TreeView

If pParentNode Is Nothing Then

Set pNode = tvwVersions.Nodes.Add (, , pVersionInfo.versionName, pVersionInfo.versionName)

Else

Set pNode = tvwVersions.Nodes.Add(pParentNode.index, tvwChild, pVersionInfo.versionName, pVersionInfo.versionName)

End If

pNode.Expanded = True

'Loop through calling this function for each child VersionInfo

'Add each VersionInfo to the Treeview of all versions

Set pEnumVersionInfo = pVersionInfo.Children

Set pVersionInfo = pEnumVersionInfo. Next

Do Until pVersionInfo Is Nothing

'Recursively add child version to tree

AddVersiontoTree pVersionInfo, pNode

Set pVersionInfo = pEnumVersionInfo. Next Loop

'Release references

Set pEnumVersionInfo = Nothing

Set pNode $=$ Nothing 


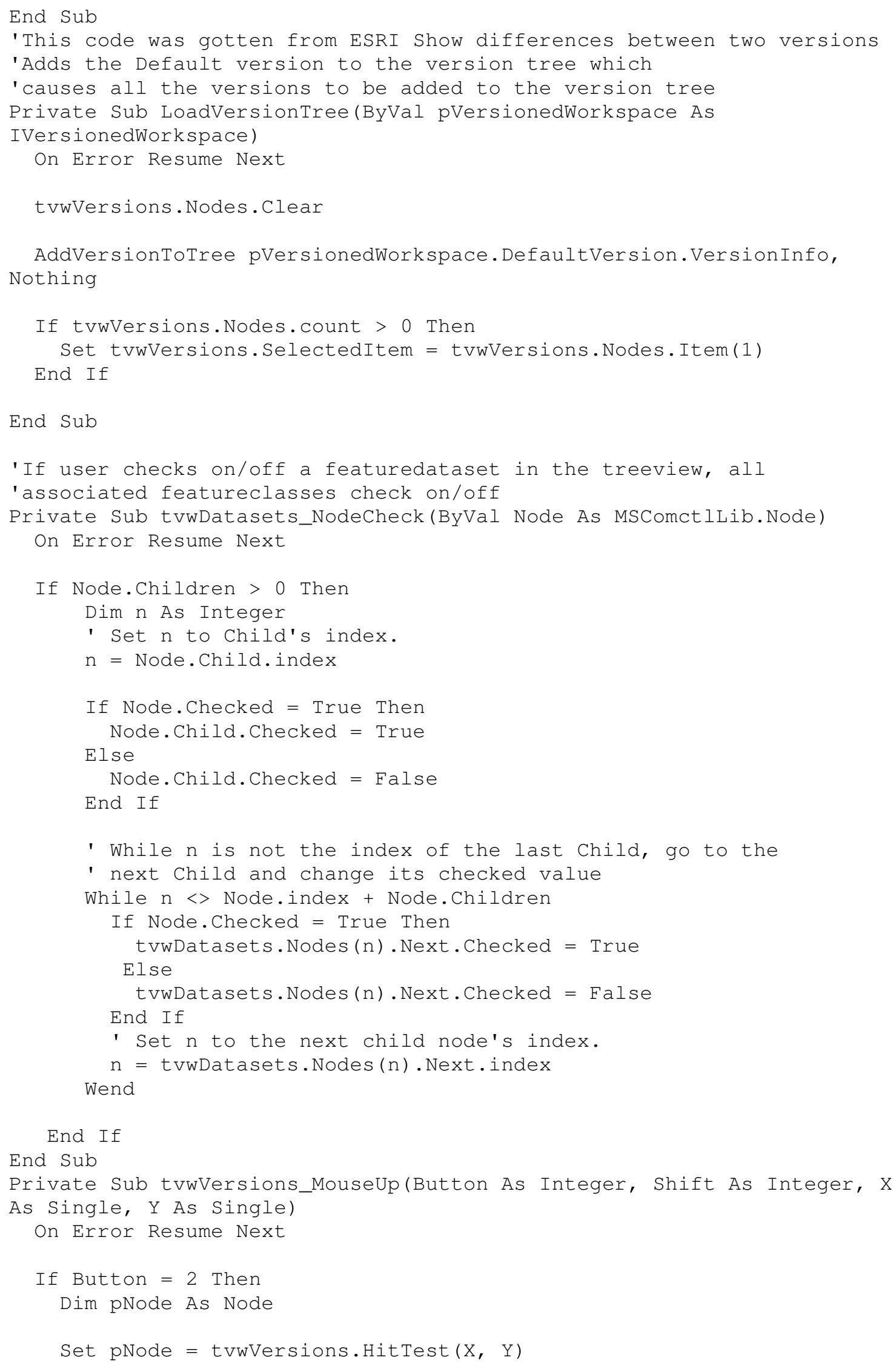




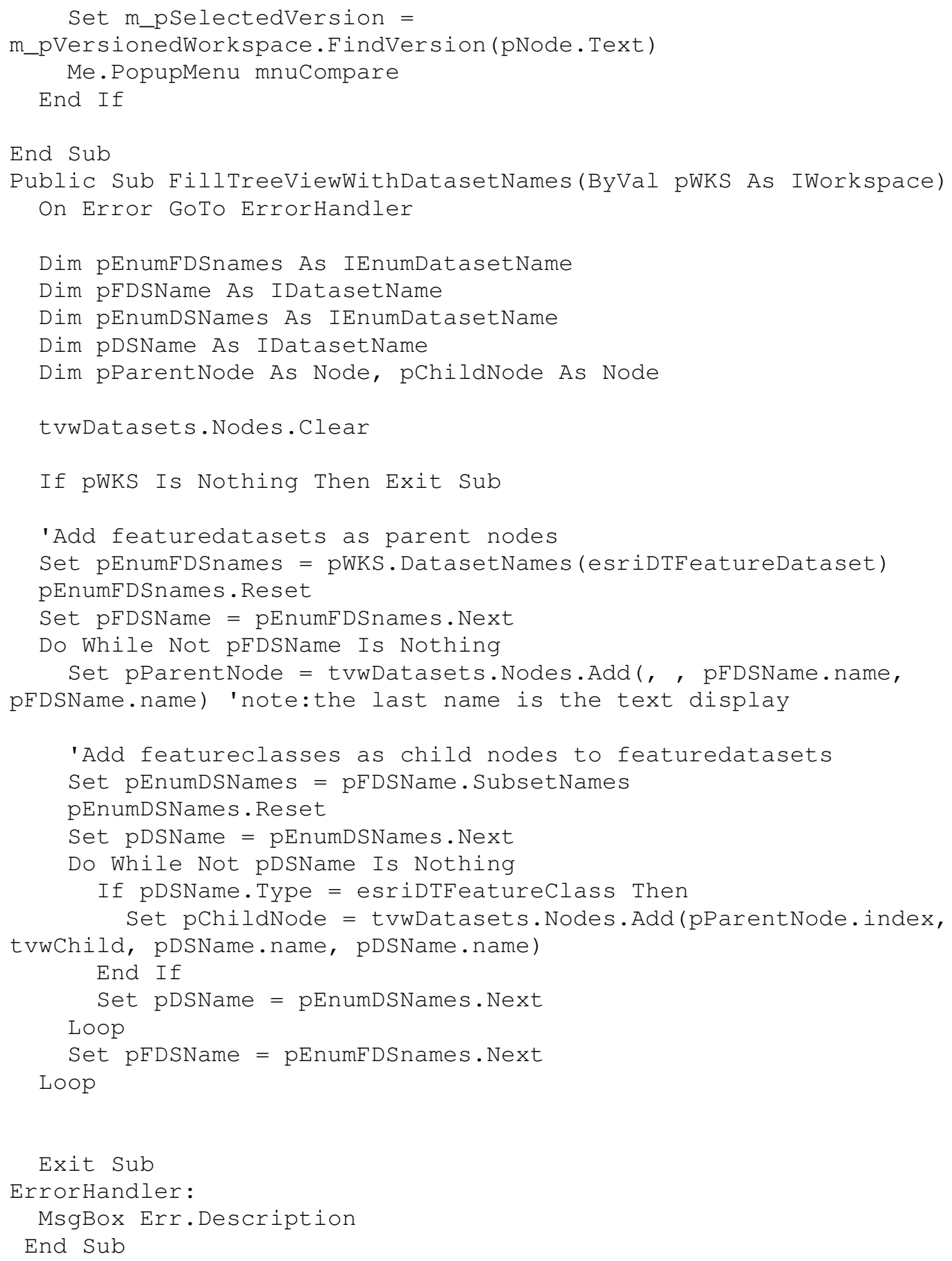

